\title{
IMPACT OF PHOTOVOLTAIC SYSTEM PENETRATION ON THE OPERATION OF VOLTAGE REGULATOR EQUIPMENT
}

\author{
A Thesis \\ presented to \\ the Faculty of California Polytechnic State University, \\ San Luis Obispo
}

In Partial Fulfillment

of the Requirements for the Degree

Masters of Science in Electrical Engineering

By

Abesh S. Mubaraki

June 2013 
(C) 2013

Abesh Sorab Mubaraki

ALL RIGHTS RESERVED 


\section{COMMITTEE MEMBERSHIP}

TITLE:

AUTHOR:

DATE SUBMITTED:

COMMITTEE CHAIR:

COMMITTEE MEMBER:

COMMITTEE MEMBER:
Impact of Photovoltaic System Penetration on the Operation of Voltage Regulator Equipment

Abesh Sorab Mubaraki

June 2013

Dr. Dale Dolan, Professor, Electrical Engineering

Dr. Ali Shaban, Professor, Electrical Engineering 


\begin{abstract}
IMPACT OF PHOTOVOLTAIC SYSTEM PENETRATION ON THE OPERATION OF VOLTAGE REGULATOR EQUIPMENT
\end{abstract}

\author{
Abesh Sorab Mubaraki
}

The growing popularity of photovoltaic (PV) generation systems leads to an increase in the number of residential and commercial grid-tied PV systems that interconnect to the distribution circuit. This affects the characteristics of the distribution circuit; for example, the assumption that the voltage profile of a radial line decreases down-stream becomes invalid because of the addition of the PV system on the line. This poses new challenges when setting the parameters of voltage regulating devices. Add to that the fact that PV systems are intermittent, especially on cloudy days, which make the line even more difficult to regulate, and the number of switching occurrences of the regulating devices increases, thus accelerating wear-and-tear to the utility's equipment.

The objective of this thesis is to develop an index which qualitatively indicates the impact of PV system(s) on operation, efficiency, reliability, and lifetime of voltage regulation equipment. Tests on the proposed index will be performed on several cases including circuits containing state-of-the art methods that integrate PV systems with minimum impact to utility equipment. Investigation of methods to further mitigate equipment wear by selecting the best interconnect point on the circuit will also be conducted to test the proposed index. The development and validation of the proposed index will entail power system modeling and simulation of distributed generation using PSCAD. The proposed index resulted from this study will provide a useful tool to allow utility companies pick the optimum locations for distributed generation to minimize their negative impact on the distribution lines as well as to determine the need for extra mitigation equipment. 


\section{ACKNOWLEDGMENTS}

When I began my educational journey at Cuesta Community College seven years ago, I had a faint idea that I wanted to be in some field of engineering. The fact that I am about to begin the next phase of my life as an electrical engineer is a testament to the great teachers that I have encountered along the way. Teachers that truly care about their students' education, and go out of their way to help students achieve their goals.

I am thankful to all my teachers for investing time and effort in me that has allowed me to reach this stage of my life. These teachers were not only from academia, but also from industry; the extremely helpful people from PG\&E and SDG\&E that took the time to answer my questions and explain things to me. I am especially grateful to my advisor Dr. Taufik. He was always able to calm me down by providing me direction and guidance when I would get overwhelmed by this thesis.

Lastly I am thankful for my wonderful girlfriend Connie, who has been by my side

and supported me through this entire journey. I look forward to starting the next phase of my life with you by my side.

Disclaimer:

SDG\&E does not endorse or accept the conclusions or results of this study. 


\section{TABLE OF CONTENTS}

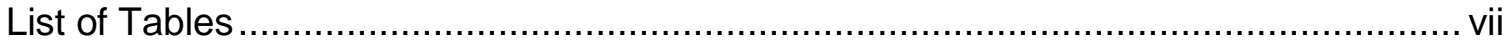

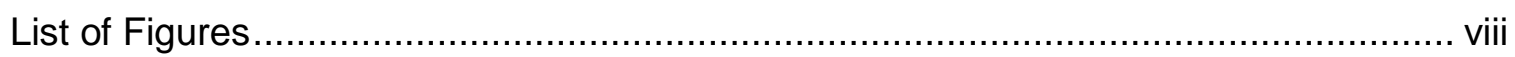

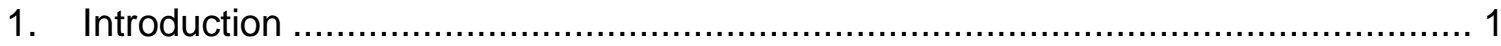

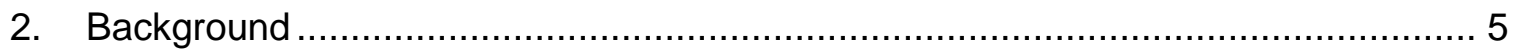

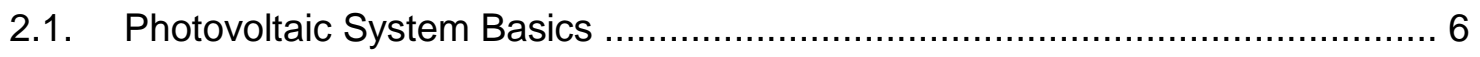

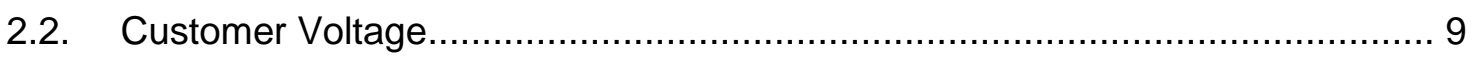

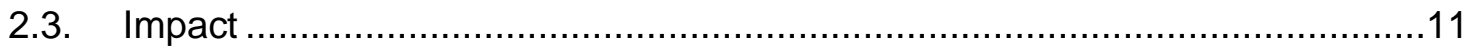

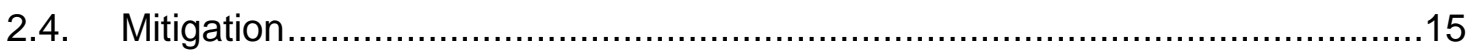

3. Existing Solution and Design Specifications …..............................................18

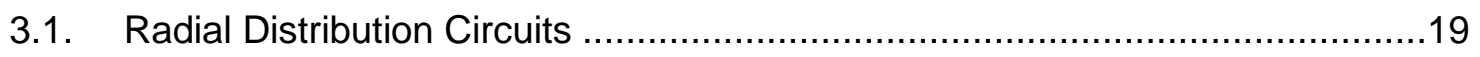

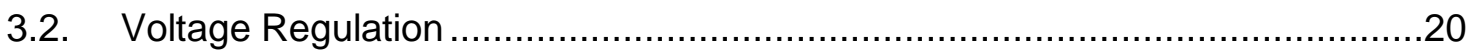

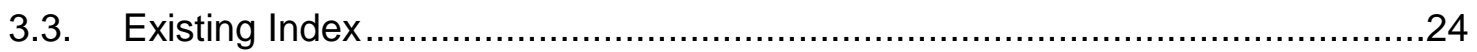

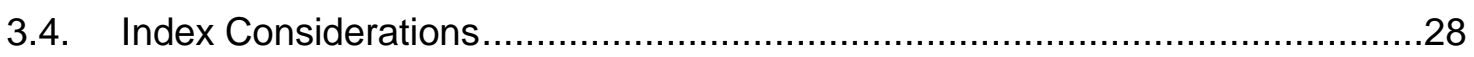

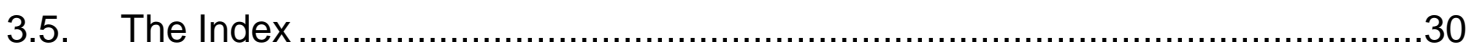

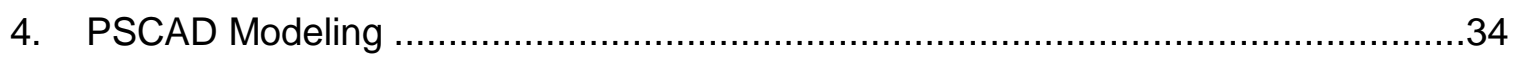

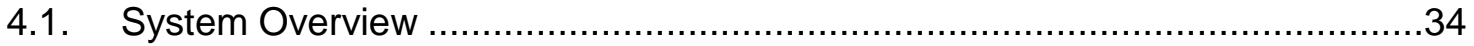

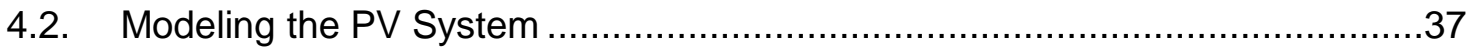

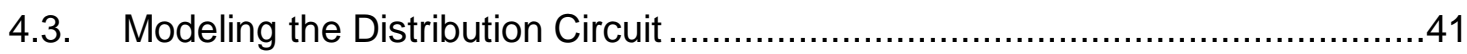

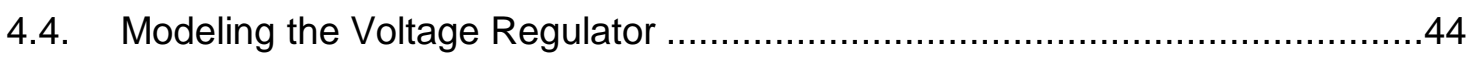

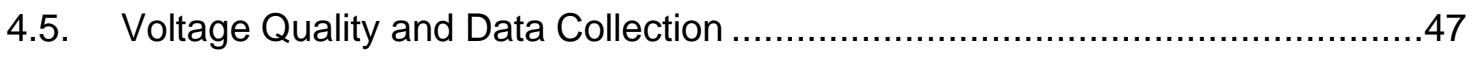

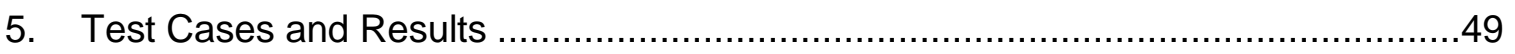

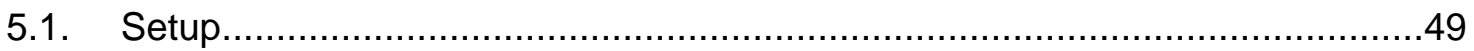

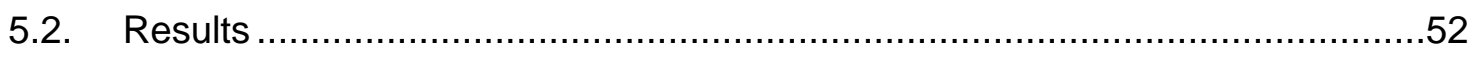

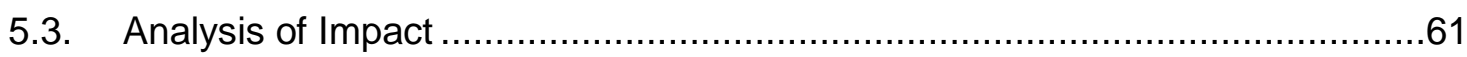

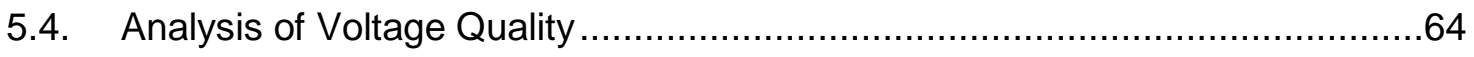

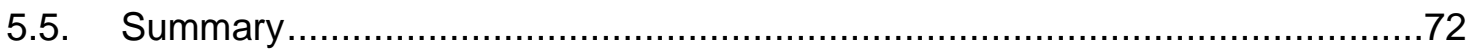

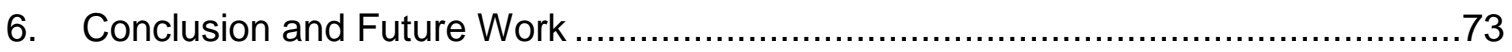

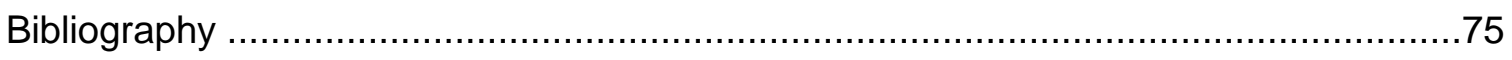

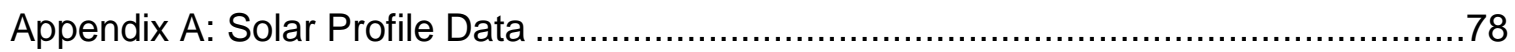

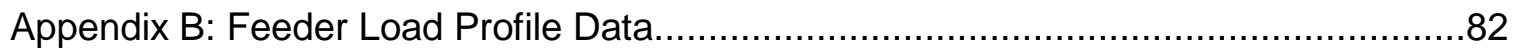




\section{LIST OF TABLES}

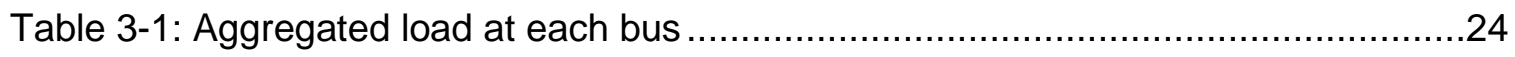

Table 3-2: Number of switching operations of devices on a radial circuit ......................26

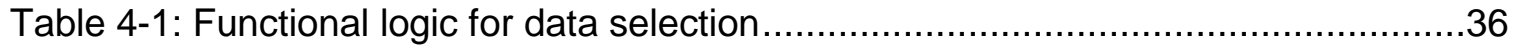

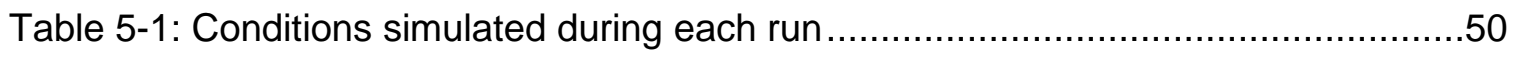

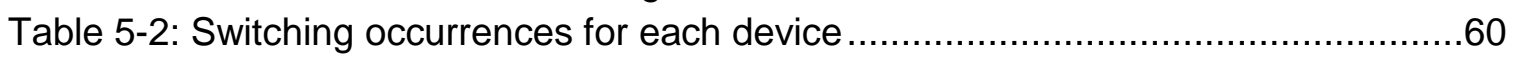

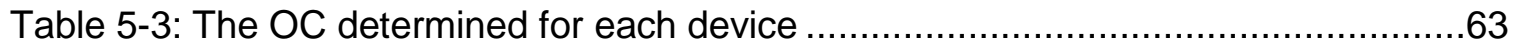

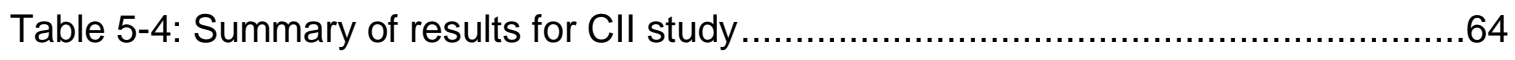

Table 5-5: Time period for over / under voltage condition .....................................70 


\section{LIST OF FIGURES}

Figure 1-1: Average cost of PV modules from 2002 to 2011 [1] ................................ 2

Figure 1-2: Annual U.S. solar thermal and PV net generation [4] ................................ 3

Figure 1-3: Utility scale electricity generation in the U.S. [6] ..................................... 4

Figure 2-1: PV system installed atop a parking structure at UCSD [8] ......................... 5

Figure 2-2: Measured solar irradiance (blue areas) and calculated clear-sky model (orange line) for a PV plant located in Tennessee [17] .............................................12

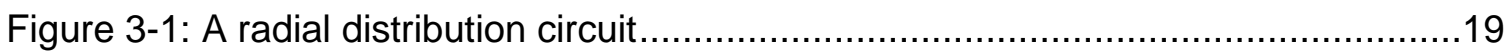

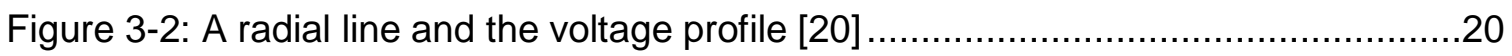

Figure 3-3: Increased load causes under-voltage condition [20] ................................21

Figure 3-4: Adding a voltage regulator to improve voltage [20] ................................22

Figure 3-5: Voltage regulator movable tap-changer contacts [23] ..............................23

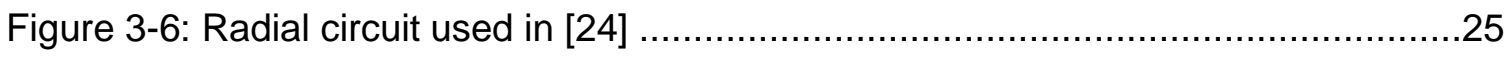

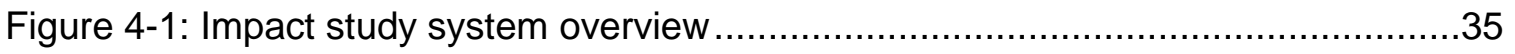

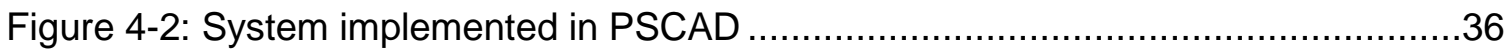

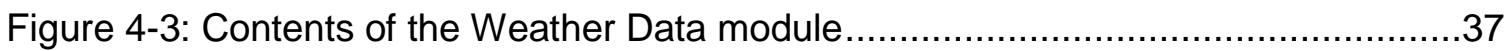

Figure 4-4: Contents of the PV System module ……...................................................

Figure 4-5: Producing a time delay before the PV system model generates power .......40

Figure 4-6: Contents of the Distribution Circuit module ............................................42

Figure 4-7: Radial circuit in PSCAD ...................................................................

Figure 4-8: Contents of the Voltage Regulator Controller module ..............................46

Figure 4-9: Contents of the Voltage Quality module..............................................4

Figure 4-10: Measuring and saving the voltage quality data ......................................48

Figure 5-1: Schematic to allow the PV system to connect to different buses ................50

Figure 5-2: Feeder load profile with no PV system connected (run 1) ..........................51

Figure 5-3: Solar profile representing a partially cloudy day .......................................52

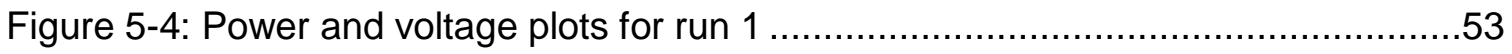

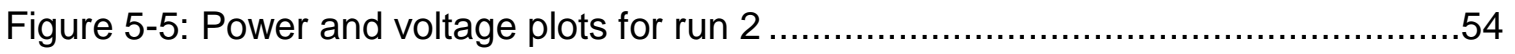

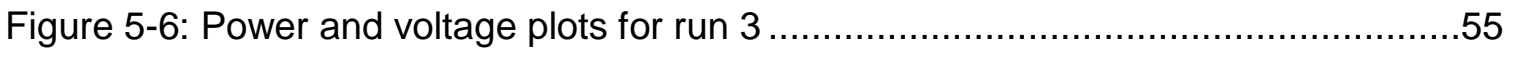

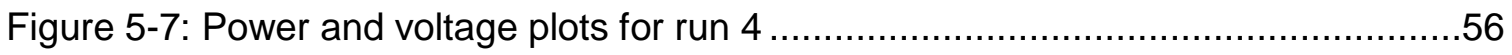

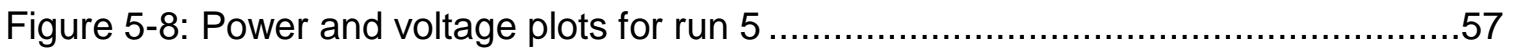

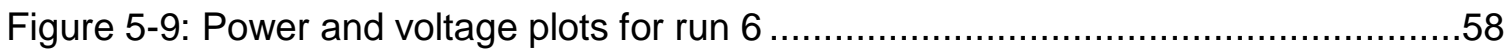

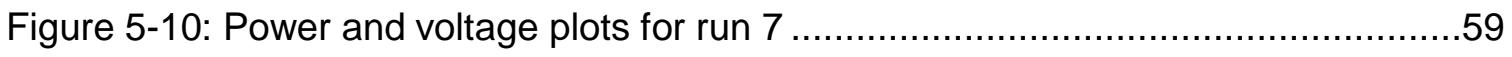

Figure 5-11: Voltage swing for no PV system connected ........................................65

Figure 5-12: Voltage swing for option 1 clear day ...................................................66

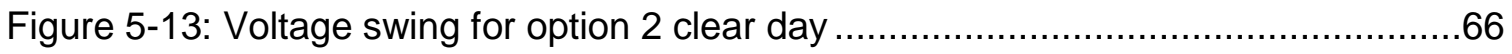

Figure 5-14: Voltage swing for option 3 clear day ................................................67

Figure 5-15: Voltage swing for option 1 partially cloudy day .....................................67

Figure 5-16: Voltage swing for option 2 partially cloudy day .......................................68

Figure 5-17: Voltage swing for option 3 partially cloudy day ....................................69

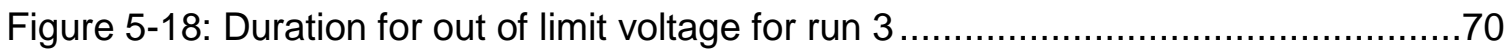

Figure 5-19: Duration for out of limit voltage for run 5 ............................................71

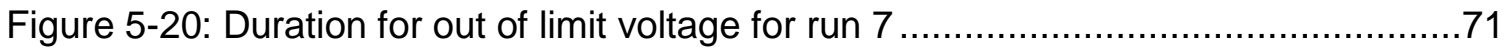




\section{Introduction}

The sun is the most fundamental source of energy for our planet; harvesting energy from other renewable sources such as hydropower, wind, and even biomass, is possible (directly or indirectly) due to the energy received from the sun. Organisms like plants are quite proficient in directly capturing and utilizing the sun's energy. Humans have been developing technologies that enable us to also capture the sun's energy. However, unlike plants, we use this energy to power the conveniences of modern life - like rotating machines, electric appliances, computers, lights, etc.

Converting the solar energy into electricity is the preferred method of utilization because of our existing infrastructure. The two popular energy conversion techniques are thermal electric and photovoltaic (PV). Thermal electricity concentrates sunlight to heat a fluid that can then power a conventional generator for example a steam turbine. One benefit of this technique is that the thermal energy can be stored for short durations and used at night or partially cloudy days. These facilities are usually utility scale plants that require relatively large area. PV systems generate electricity directly from sun light by using the energy from photons to create a flow of electrons. The modular nature of PV panels allow for the system to be easily scaled based on the available physical space. This makes it the preferred choice for residential and commercial scale. Note that solar thermal is also used on a residential/commercial scale, but instead of generating electricity, the heat is directly used for hot water and space heating. In fact, since PV panels have higher efficiencies at lower temperatures, extracting the thermal energy from the panel to be used for heating applications is being actively researched. 


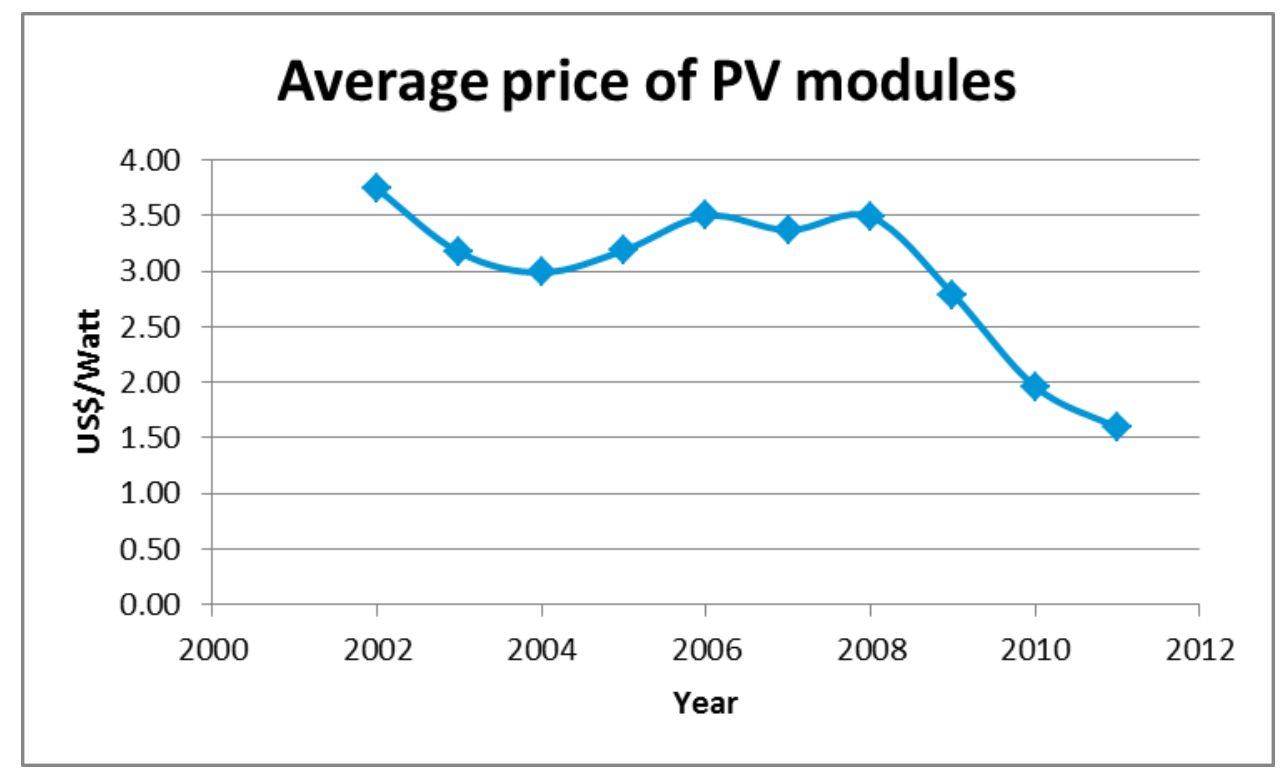

Figure 1-1: Average cost of PV modules from 2002 to 2011 [1]

Over the years the cost per watt of PV systems has decreased. Figure 1-1 shows the trend in the average cost per peak watt-DC of PV modules (also called panels) over the past decade. We see that the cost has been reduced by half from $3.74 \$ /$ Watt in 2002 to $1.59 \$ /$ Watt in 2011 ; the price is not adjusted for inflation which would make the cost reduction even more pronounced. "As of 2011, the cost of photovoltaics has fallen well below that of nuclear power and is set to fall further" [2]. Along with the PV modules, the cost of the rest of the system (inverters, mounting hardware, etc.) is also decreasing. "Photovoltaic (PV) system prices have dropped by $50 \%$ over the last five years, which has led to remarkable growth in solar system installations. As of 2012, about $6 \mathrm{GW}$ were installed in the United States and more than $90 \mathrm{GW}$ worldwide. [...] In the United States, prices for installed PV systems at the utility scale (over $10 \mathrm{MW}$ ) average US\$3 per watt. [...] [The U.S. Department of Energy's] Sunshot Initiative targets by 2020 of US\$1.00/W for utility-scale PV systems, US\$1.25/W for commercial rooftop PVs, \$1.50/W for residential rooftop PVs" [3]. The standardization of mounting hardware and connectors allows for faster setup thus reducing the system installation cost. 


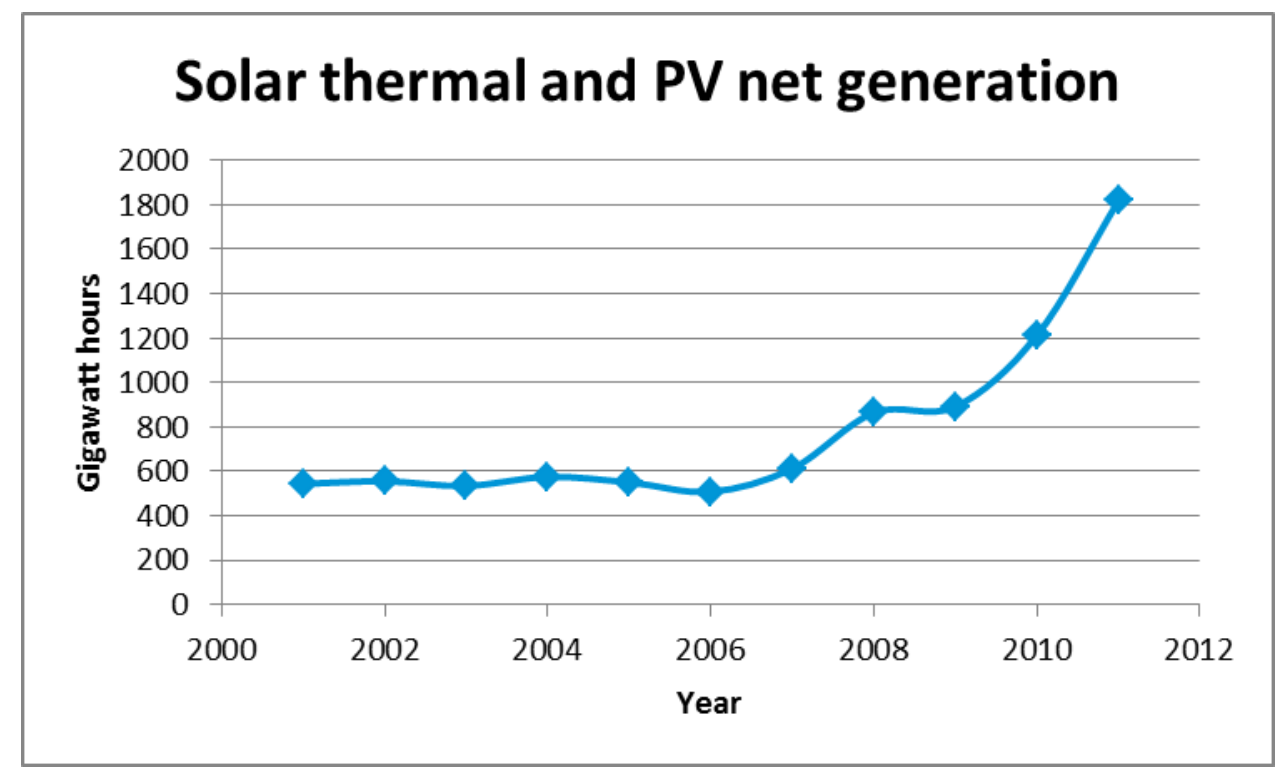

Figure 1-2: Annual U.S. solar thermal and PV net generation [4]

There have been and continues to be various incentives and rebate programs offered to install PV systems; up-to-date information about the incentives and policies by location in the U.S. can be found at: http://www.dsireusa.org/. Due to this, the utilities are experiencing an increasing number of residential and commercial PV systems wanting to connect to its distribution circuits. Figure 1-2 shows the annual net energy generated in the U.S. from solar resources. There was a $50 \%$ increase in this energy generation from 2010 to 2011 ; however, this is still only $0.044 \%$ of the total energy generated from all sources, so there is ample room for growth for these generation methods [4]. The potential for growth can be seen by observing how little is currently contributed by solar shown in Figure 1-3. The figure represents utility scale generation; however, an increase in utility scale PV systems will aid in lowering the system cost for residential and commercial scale systems too.

California's Renewable Portfolio Standard (RPS) requires investor-owned utilities (and several other entities) to have $33 \%$ of total energy procurement from renewable 
energy resources by 2020 [5]. To achieve this, the utilities need to approve the maximum number of interconnect requests from renewable generation sources without compromising system stability, and while minimizing negative impacts to voltage quality and utility's assets. One such renewable generation source is PV systems, and interconnection requests come in sizes from a few kilowatt residential systems to hundreds of gigawatt utility scale solar farms. Larger systems may be connected to the transmission circuit; however, smaller systems up-to a few gigawatt are generally connected to the distribution circuit. Despite the benefits provided by grid-tied PV systems, large scale grid-tied PV systems may also introduce problems in the power system they are attached to. The following chapter explains this.

\section{Sources of Electricity Generation, 2011}

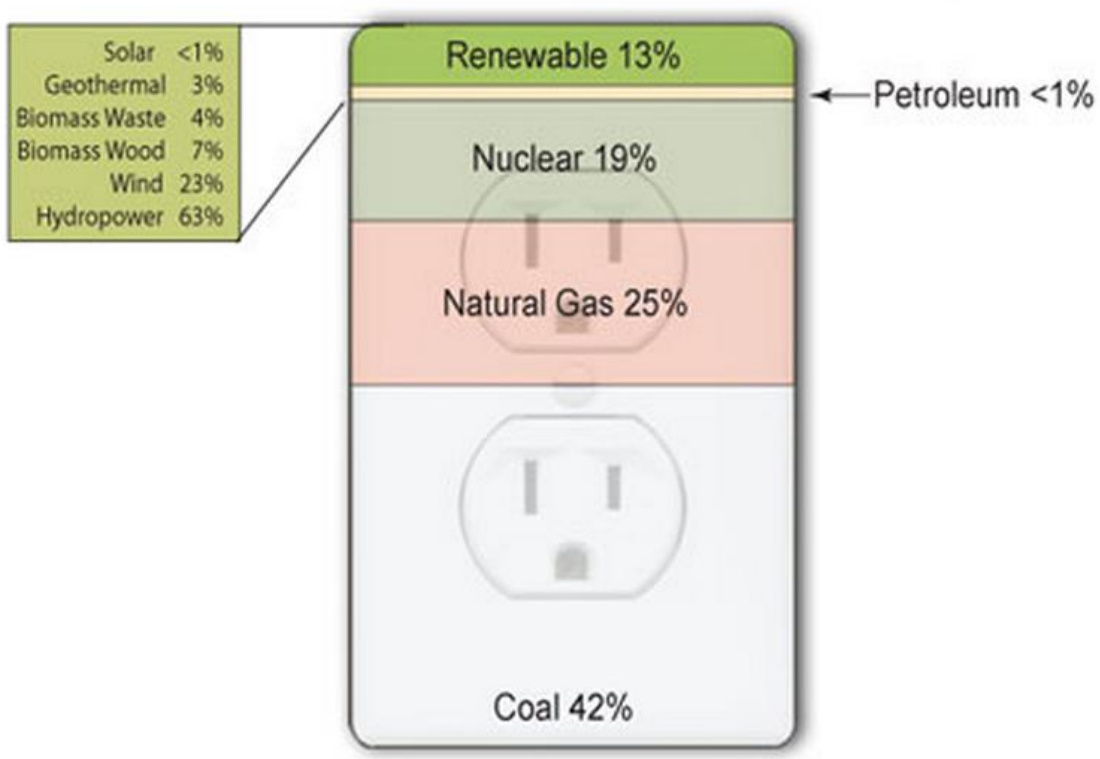

Figure 1-3: Utility scale electricity generation in the U.S. [6] 


\section{Background}

For a residential customer with a desire to generate electricity, installing a PV system is a great choice. The system is clean, quiet, almost maintenance free, and takes up very little usable space. A small amount of logically placed PV generations can reduce considerable losses on a feeder with high loss; this is beneficial to the utility [7]. However, larger PV systems, or clusters of smaller systems in the same vicinity, present the utility with certain issues that need to be addresses. In this chapter we will explore some negative impact of grid connected PV systems and the mitigation options available to the utility.

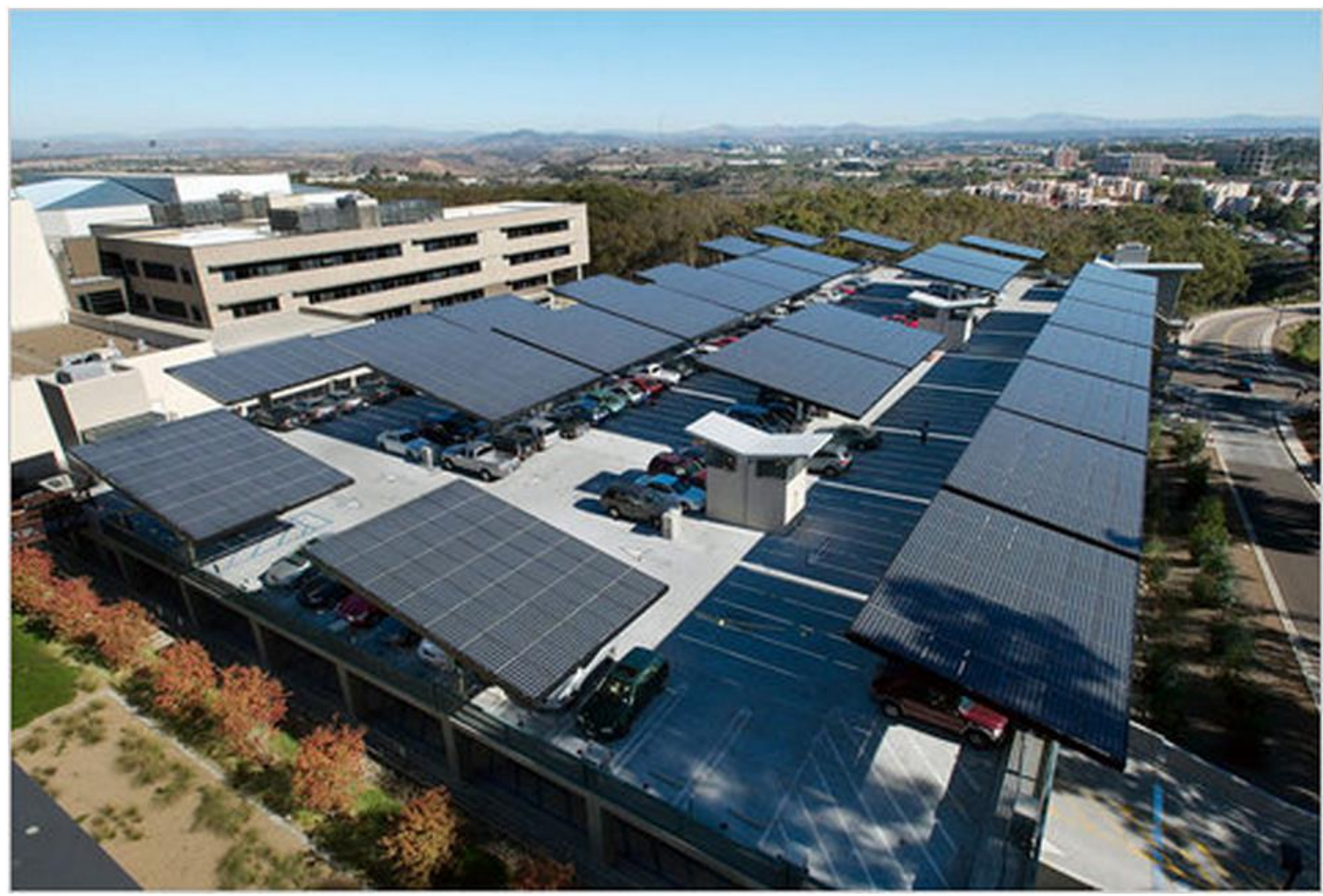

Figure 2-1: PV system installed atop a parking structure at UCSD [8] 


\subsection{Photovoltaic System Basics}

In order to realize the impact of the PV system, it is beneficial to first be familiar with some of the basic sub-systems of the PV generation plants. Generally most grid connected PV systems can be electrically split up into the following blocks:

- PV Array: The array is the most visible component of the PV system, and it takes up the most (surface) area. However, the space occupied by the array is not always useful for much else, such as in residential rooftop installation; in fact, sometimes the array can serve a secondary useful purpose, such as providing shade when used in place of conventional parking lot shading structures (Figure 2-1). The array is the collection of all the panels of the PV system. A number of panels are connected in series to form a string; the number of panels per string and the number of strings in an array is determined by the desired topology and the type of equipment selected.

- DC Filter and Maximum Power Point Tracker (MPPT): The DC filter is a capacitor bank that reduces the ripple seen by the array due to the switching of the power electronics and also provides a stable input voltage to the MPPT. A MPPT is a DC to DC converter that operates at a point of the PV panel's/string's I-V curve that draws the most power. The nominal input of the MPPT is used to determine the number of panels in a string. However, the MPPT can operate over a wide input voltage range above and below its nominal; this is crucial since it accounts for variations in the string's output due to shading, dust, temperature, etc. Ideally there is a MPPT for each string, but it is possible to parallel multiple strings using a junction box and then use a MPPT; this method reduces cost and performance. This block might be combined with the inverter in the same enclosure to provide a more convenient form-factor. 
- Inverter: The output of the MPPT via a DC link capacitor is the input to the inverter. The inverter is a DC to $A C$ converter that can be designed using various isolated and non-isolated topologies. Due to the grounding requirements in the U.S., the isolated topology is most commonly used. The output of the inverter is filtered and conditioned until it is suitable to connect to the grid. There is a subset of inverters called microinverters that is attached to each individual panel such that each panel is a self-contained PV system. The main drawback to microinverters is higher initial cost, but this is offset by better energy returns in the long run since each panel is operating at peak performance due to individual MPPT [9]. Also a partially shaded panel does not affect the performance of an entire string.

- Energy Storage (optional): Grid connected systems draw power from the grid when the load exceeds output of the PV system, therefore energy storage is not a necessity. Nevertheless adding energy storage to a grid connected system does provide some benefits that can justify its cost. One benefit is that with proper equipment and safety features, it is possible to create a local island for sensitive loads such as hospitals during momentary loss of grid power, or until backup generators come online during an extended unexpected outage. Another important benefit of energy storage is the ability to slow the power ramp down rate caused by intermittent cloud shading - this is one option considered when trying to mitigate the negative impact of the PV system.

- Balance of System: There are various other devices that are part of the system for reasons ranging from safety to increased system features; this includes breakers, fuses, surge protectors, grounding devices and equipment, GFID, sensing and measuring equipment, displays/indicators, communication ports etc. 
Most inverter topologies are versatile and can operate over a range of power factor by reprogramming or changing the controller. This implies that most grid connected inverters are capable of providing real and reactive (capacitive or inductive) power. PV systems connected at the distribution level follow the IEEE 1547-2003 standard, which specifies that the distributed resource (the PV system in our case) cannot actively regulate the voltage at the point of grid connection [10]. "IEEE 1547 is only concerned with conditions at the point of utility connection[...] It should be noted that the voltage values apply to the point of utility connection, also known as the point of common coupling (PCC) for the inverter. If the inverter is located some distance from the PCC, there may be voltage drop on the line between the inverter and the PCC. If so, compensation can be made at the inverter output, because the inverter output voltage in these cases will be higher than the voltage at the PCC in order for the inverter to deliver power to the $P C C^{\prime}[11]$.

If the PV system has a significant impact on the local power system, then it's possible for the system to operate at a specific non-unity power factor to minimize effect [12]. However, since the utility tariff structure generally only compensates PV system owners for the real power produced, most inverters are set to supply power at or close to unity power factor.

To begin to understand the problems caused by the grid connected PV system, we need to understand the obvious: PV panels generate electricity only in the presence of light. The implication is that when the light on the panel (or irradiance ${ }^{1}$ ) is reduced then the output power of the panel is also reduced; it is important to realize that this is not a linear relationship. "Even a small amount of shade on a PV module can significantly reduce the module output current" [11]. Assuming a correctly sized inverter, the amount

\footnotetext{
${ }^{1}$ In this thesis, irradiance is the measure of solar power on a particular plane measured in $\mathrm{W} / \mathrm{m}^{2}$.
} 
of real power the inverter can supply to the grid is the power supplied by the PV array minus system losses. Because of MPPT the output power approximately tracks the irradiance on the plane of the array, and this can cause large variations of output power in relatively short periods of time by natural phenomena such as passing clouds. Such variations can cause issues in the local power system that will be discussed in the following sections.

\subsection{Customer Voltage}

An average customer expects the grid to provide a constant rms voltage - to behave like an infinite bus or ideal source that has a steady voltage no matter how much power is being supplied or absorbed - but this is not the case. The reality is that electrical conductors have some impedance; for this reason, even if the voltage at some point of the power system is kept steady, the voltage at another point downstream will vary with the change in power consumption at that point and further downstream.

The intrinsic properties of the power system make it impractical to try to maintain a constant voltage at a particular point, so typically the voltage changes throughout the day. The goal here is to keep the voltage swings within tolerable limits and as close to the nominal voltage as possible. The National Electrical Manufacturers Association (NEMA) publishes the ANSI C84.1standard that "establishes nominal voltage ratings and operating tolerances for 60-hertz electric power systems above 100 volts" [13]. The standard requires the deviation at PCC to within $5 \%$ of the nominal system voltage; between $114 \mathrm{~V}$ to $126 \mathrm{~V}$ for a 120 volt base [12] [14]. "Most regulatory bodies and most utilities in America follow the ANSI voltage standards" by designing power systems to keep service voltages within the tolerance, and limit the occurrence of voltage 
excursions [14]. Some utilities may design a feeder to more stringent standards in order to meet the needs of a customer with higher voltage quality requirements, such as a hospital or data center.

In order to control the voltage, various types of voltage regulating devices are used. The following are some of the devices used for voltage regulation [14]:

- Substation load tap-changing transformers (LTCs)

- Substation feeder or bus voltage regulators

- Line voltage regulators

- Fixed and switched capacitors

The operating principal of the first three can be thought of as adjusting the turns ratio of a transformer to get the desired voltage level. Whereas, the switched and fixed capacitor function by improving the power factor and thus reducing the voltage drop along the power lines; generally the loads are of a lagging power factor (inductive), hence the use of capacitors for power factor correction. All but the fixed capacitors perform some sort of switching/tap-changing action to change the voltage level. These devices may sense voltage, current, or power factor to determine when to switch, or they may switch at certain times during the day/week depending on the expected load profile, as is typically the case for switched capacitor banks.

Normally the devices have a time delay before switching to confirm that the voltage is truly out of bounds rather than a transient fluctuation; this time delay also prevents excessive switching actions and minimizes equipment wear. The addition of PV systems increases the difficulty of controlling the voltage and can negatively impact the equipment's' service life as we shall see in the next section. 


\subsection{Impact}

Customers' voltage quality decreases when the voltage swings out of acceptable limits, and this could damage equipment that cannot handle over/under voltage operation. PV systems introduce uncontrollable variability into the distribution system it interconnects with. The magnitude of the variability depends on several factors such as PV system size, local weather patterns, electrical location of interconnect on the circuit, line impedance, type and size of other loads on the circuit, etc. The power requirements of a feeder vary with time, and this variability often gradual and somewhat predictable. For example a particular residential feeder might be expected to consume peak power in the evening hours. Much of the utility's equipment presently in place is designed for and able to correct the effects of such variation. In contrast, the variation introduced by PV systems is rapid and sporadic, hence if such variation has a large magnitude, then it can have a negative impact on the power system and the equipment connected to it.

When discussing PV systems on a distribution network, a metric that is often referenced is the penetration level; it is usually defined as [15]:

$$
\text { Penetration level }=\frac{P V \text { system rated power output }}{\text { Peak circuit load }}
$$

The penetration level is typically expressed as a percent value. In circuits with low penetration levels, smaller PV systems will have negligible impact; small is a relative term, in this case it can be thought of as a residential PV system that offsets most of the home's load. However, even at low penetration levels, a large PV system (250kW for example) can have an adverse effect on the voltage quality depending on the characteristics of the distribution circuit [15]. With an increase in penetration level, such effects are further aggravated, and other issues such as system stability also need to be 
considered. A simulation with $40 \%$ penetration of distributed generation on a feeder with motor loads showed that a voltage collapse is possible [16].

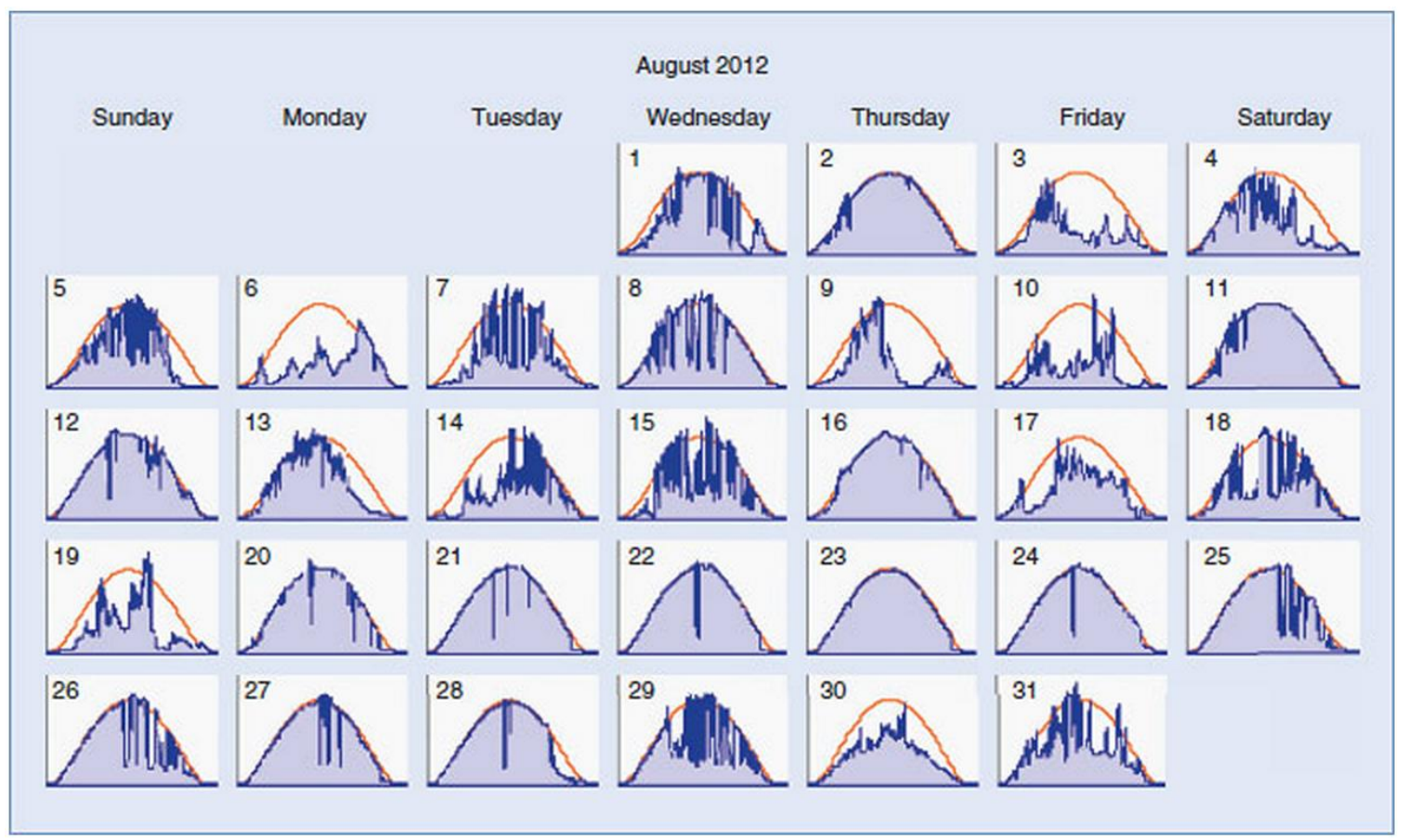

Figure 2-2: Measured solar irradiance (blue areas) and calculated clear-sky model (orange line) for a PV plant located in Tennessee [17]

The most significant source of uncontrollable variability in PV systems is a result of changing irradiance on the array. This is usually a result of shading caused by moving clouds. Figure 2-2 shows the irradiance at PV plant in Tennessee. The irradiance was measured and averaged over one minute intervals using pyranometer in the plane of PV array for the month of August 2012. This provides some insight into the extent that the output of a PV system can fluctuate, and do so quite rapidly. "Typical automatic line equipment, such as capacitors and voltage regulators, has time delays ranging from 30 to 90 seconds" [15]. Such slow devices perform satisfactorily in distribution networks consisting of loads that are somewhat predictable based on time of day and season of the year. However, the intermittency of PV systems causes fluctuations that are not 
predictable, and could occur on a time scale that is too fast for such devices with traditionally long delays to react.

Due to uncontrolled variability, the power output of the PV system can increase or decrease quickly. The rate at which the output power changes is called the ramp rate, and for PV systems it is around $10 \%$ to $15 \%$ per second [17] [18]. This is very high even when compared to another renewable source like wind generation which has ramp rates of about $10 \%$ per minute [17]. The reason for this is that unlike wind generation, PV systems do not have any rotational inertia which dampens sudden changes; PV systems use MPPT to constantly push out the maximum power out so as soon as the irradiance changes the output power follows.

An intuitive way to grasp the impact is to view the PV system as displacing (offsetting) some load on the circuit. The voltage regulation equipment has auto-adjusted to compensate for the voltage drop along the line due to the existing load conditions. A moving cloud shades the array, and the output of the PV system significantly reduces in a matter of seconds; the load that was being supplied by the PV system suddenly draws power from feeder. If the load displaced was large (i.e. large PV system), then the additional current being drawn by the load could cause the voltage to drop below allowable limits. The voltage regulating devices sense this and switch taps after a preset delay to bring the voltage up. However, in a few minutes the cloud moves away and the PV system's power output increases. Now the feeder's power consumption drops, and the reduced current flow through the wires leads to a reduced voltage drop resulting in high voltage condition, and causes the regulating equipment to switch taps to the original position after the delay. This scenario can occur multiple times a day on a partially cloudy day, thus leading to significant increase of switching frequency and decrease in voltage quality. 
The size of the PV system compared to other loads on the circuit gives a good first estimation of the impact the PV system will have on the power system, but that is not the only factor that needs to be considered. A cluster of smaller PV systems located in close proximity could have a similar impact as one large PV system. There are various other factors that need to be considered to determine the severity of the impact that the PV system will have; these are [15]:

- The electrical characteristics of the electrical system at the location of interconnect. Generally the impedance will be higher for interconnects further downstream. This leads to an increase in the voltage fluctuation effects to the surrounding loads due to passing clouds, and can also cause the steady state voltage levels to be high.

- Daily load profile with a sizeable solar injection. The greater the penetration level, the more it will change the feeder profile which can increase the number of switching operations performed by voltage sensing line equipment.

- Low weekend load. Most distribution systems were designed for power flow in one direction - from substation to the load. This is no longer the case when a PV system is added to the circuit, especially on the weekends when many circuits tend to be lightly loaded. Under such conditions power can flow back into the feeder and can cause the voltage on the feeder to be high.

- Large systems with load tap changer (LTC) on the substation transformer secondary. The stitching operations of such systems can increase, thus requiring more frequent maintenance.

- Large solar systems installed close to regulators, capacitors, and reclosers. The device settings will need to be recalculated, and in some cases the devices will need to be relocated to a better suited place in order to continue 
to provide reliable power to customers. Once again the switching operations can increase - significantly in some cases - and the maintenance cost increases.

The customer interconnecting the PV system to the distribution circuit introduces uncontrollable in the load profile of the circuit, which in turn leads to lower voltage quality for other customers on the circuit. However, the utility is responsible to hold the voltage within the allowable limits; in order to do so, the utility can implement several mitigation options that will be discussed in the next section.

\subsection{Mitigation}

There are several different options that the utility can choose from to mitigate the impact caused by PV on a particular circuit. Here are some options:

- Reconductoring: Upgrading to better/larger conductors is frequently done by the utility to accommodate more load on a circuit. This may address some of the issues caused by the PV system, but according to the study in [15], reconductoring spread voltage fluctuations under certain conditions further into the system. Therefore reconductoring might not always be the best option [15].

- Energy storage: The most common choice for energy storage is in the form of batteries. This is a subject that is being actively researched for a couple of reasons: (a) battery technology is improving significantly, but data on the effect of utilizing batteries for such applications (rapid charging/discharging) is limited, especially for the newer battery chemistries; (b) there is an expectation of an influx of electric cars wanting to connect to the grid and this could provide significant distributed energy storage. The energy storage option can technically be separated into the following two options: 
Energy storage at the PV site: By installing energy storage at the PV site, more specifically interconnecting it with the PV system, it is possible to control and slow down the ramp down rate when the array's output decreases due to cloud shading. Limiting the ramp up rate does not require any energy storage because the inverter can be programmed to gradually increase output power instead of pushing the MPPT power straight through. Controlling the ramp rates is akin to dampening the variability thus minimizing fluctuations of shorter duration which can improve voltage quality and reduce the switching operations on the regulators.

- Energy storage away from the PV site: It is not always possible to add energy storage at the PV system location, yet energy storage can be beneficial to the circuit at another location, perhaps the substation, or distributed throughout via electric cars plugged into the circuit. In this situation the energy storage can be used for what is often called peak shaving, where it absorbs power during periods of low load or excess power and supplies power when load increases. In this case, the electrical location the PV system and energy storage will play a role in how effective the mitigation will be.

- Reactive power source: There are several devices using power electronics that control reactive power to provide voltage support and power flow control on the transmission system. With the reducing cost of technology it can make economic sense to install similar devices on the distribution system. Due to the use of power electronics, such devices can react to circuit conditions on time-scale that may be impossible for the mechanical systems in traditional voltage regulators, and can do so with minimum equipment wear. 
- Regulator settings: It is possible to improve voltage quality by increasing the sensitivity (tighter bandwidth) and/or reducing the time delay setting, but this will lead to more frequent switching thus reducing the device's service life [14].

- Updating standards: By changing the control scheme, inverters can also function as reactive power sources thus helping improve the voltage quality. "As electric power systems transition to higher levels of distributed generation, one of the most economical solutions is to allow the inverters to help regulate voltage locally" [15]. This option is not in the same category as the other options listed, but it is nonetheless an important option that various entities are researching.

Each option has some trade-offs, and depending on the specific circuit, it is possible to have a 'best' mitigation solution. However, since technology (and problem) is new, the trade-offs and benefits are not always well documented or obvious. This makes it a risky investment for the utility since it is difficult to determine the optimum solution for a particular situation, and the (electrical) location placement that would maximize the mitigation benefits while also making the most economic sense.

Thus developing an index that quantifies the impact of the PV system and mitigation options to the utility's equipment and the customers' voltage quality would prove to be a beneficial tool to the engineers making the decision regarding PV interconnects and mitigation options. The purpose of this thesis is to create a tool that will allow utility engineers to compare the effectiveness of the various mitigation options and make decisions that provides the best bang for the buck. This will be discussed in the next chapter. 


\section{Existing Solution and Design Specifications}

As engineers, we continuously update old metrics and create new ones as technology progresses. Such metrics allow us to track and compare various parameters of interest, for example: various types of efficiencies, computational speed, transistor density, etc. The variability of PV system is in two forms: predictable, caused by day and night, and un-predictable, caused usually by moving clouds. PV systems are also uncontrollable, because under the current standards, the PV system is constantly pushing the array's output to the grid; this implies the lack of spinning reserve. Therefore many of the metrics used to quantify performance of conventional power plants are not applicable to a non-dispatchable generation resource like PV systems. With the number of PV systems interconnecting to the grid increasing, there is a lot of research being done to define various metrics as applicable to PV systems.

However, much of the research effort is focused on metrics that can be used to quantify various aspects of the PV system, whereas little is being done in way of quantifying the effects on the utility's equipment. Having such a metric is important because it provides the utility a tool to allow for an optimum level of PV penetration, and also to compare the effectiveness of various mitigation options along with determining the ideal placement along the circuit. Thus far only one such metric has been defined; it serves as a starting point, but there is much room for improvement. In this chapter the existing metric will be examined, and a more versatile metric will be defined. 


\subsection{Radial Distribution Circuits}

Circuits that consist of only one path from the customer to the substation are called radial circuits; a simplified example of a radial circuit is shown in Figure 3-1. Radial lines may split downstream in two or more paths called laterals. The loads can be single phase or three phase, and are typically connected to the circuit through a step down transformer that reduces the voltage from a distribution level (4kV and higher) to a service level (480V and lower). Most residential and many commercial connections tend to be single phase, whereas industrial and agricultural customers are supplied power via three phase connections. PV systems are connected to the circuit in a similar fashion as a load would be. "Currently, about $80 \%$ of PVs is connected to the distribution grid" [17]. Larger PV systems are connected to the circuit as a three phase system that is balanced under normal operations.

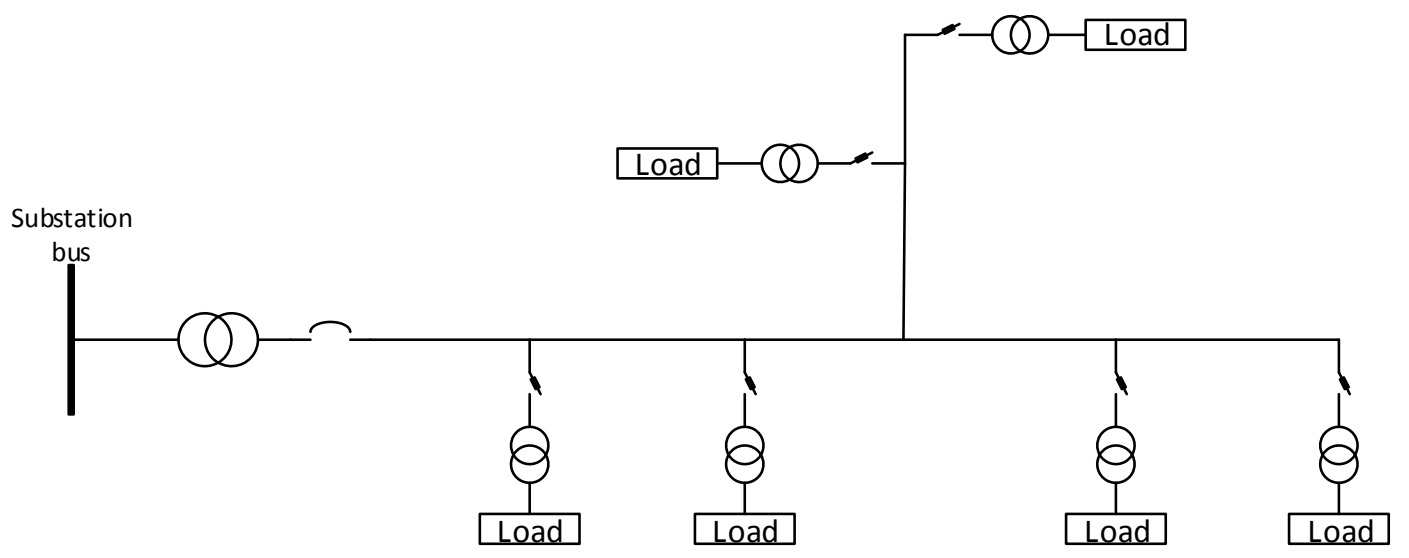

Figure 3-1: A radial distribution circuit

The cases studied in this thesis will focus on radial circuits because over $80 \%$ of worldwide distribution networks consist of radial circuits [19]. Often times even loop circuits consist of a normally open tie switch, thus operating as a radial circuit. Also due to space requirements, large systems are typically located in non-urban areas where 
radial circuits are very prevalent. However, it is possible to use the metric defined in this chapter to study the impact of a large PV system in an urban area, interconnected to a mesh distribution network, but accurate circuit model and the load profile needs to be created.

\subsection{Voltage Regulation}

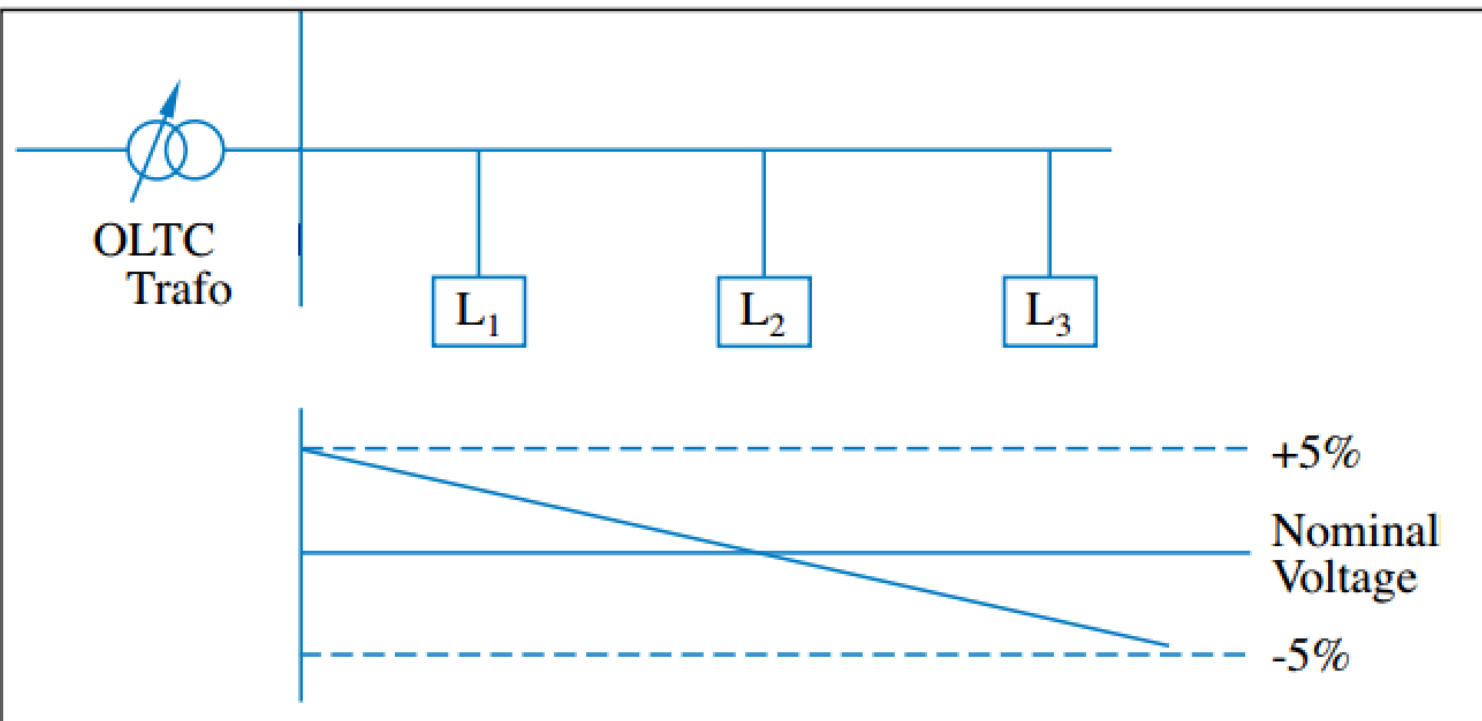

Figure 3-2: A radial line and the voltage profile [20]

To understand the need and function of voltage regulation devices, the following simplified example is presented. Figure 3-2 consists of a radial circuit supplying three loads and the voltage profile along the line. The substation transformer's on-load tap changer adjusts the voltage to be above the nominal voltage, yet within the allowable limits, and the voltage decreases in the downstream direction because of the line impedance. This being a simplified example, the voltage drop is assumed to be linear for the length of the line, this will almost never occur in actual circuits. The voltage at the end of the line is below the nominal voltage but still within the specified limits. If the load 
on the circuit increases, as shown in Figure 3-3, then the voltage drop along the line also increases and some of the loads are being supplied by a voltage that is below the allowable limit. This problem cannot be solved by increasing the voltage at the substation, as this would negatively impact the first customer on the circuit. In order to solve this problem a voltage regulator $\left(R_{2}\right)$ is placed at an appropriate location on the circuit to boost the voltage and maintains it within the limits for the entire length of the line; this can be seen in Figure 3-4. "These voltage-regulating transformers are commonly capable of increasing or decreasing the distribution voltage up to $10 \%$, usually in multiple steps of either $0.625 \%$ or $1.25 \%$ per step" [21].

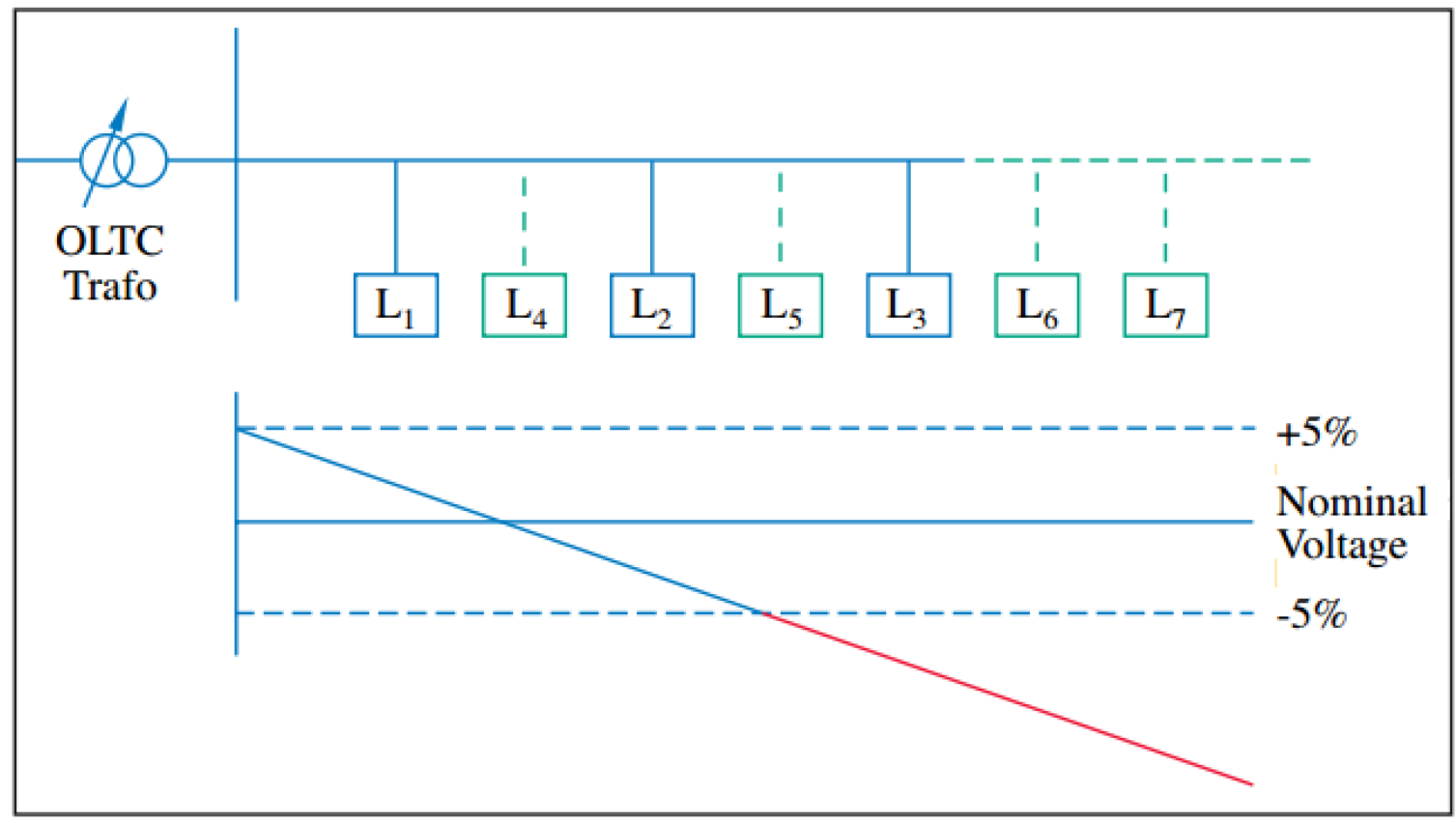

Figure 3-3: Increased load causes under-voltage condition [20] 


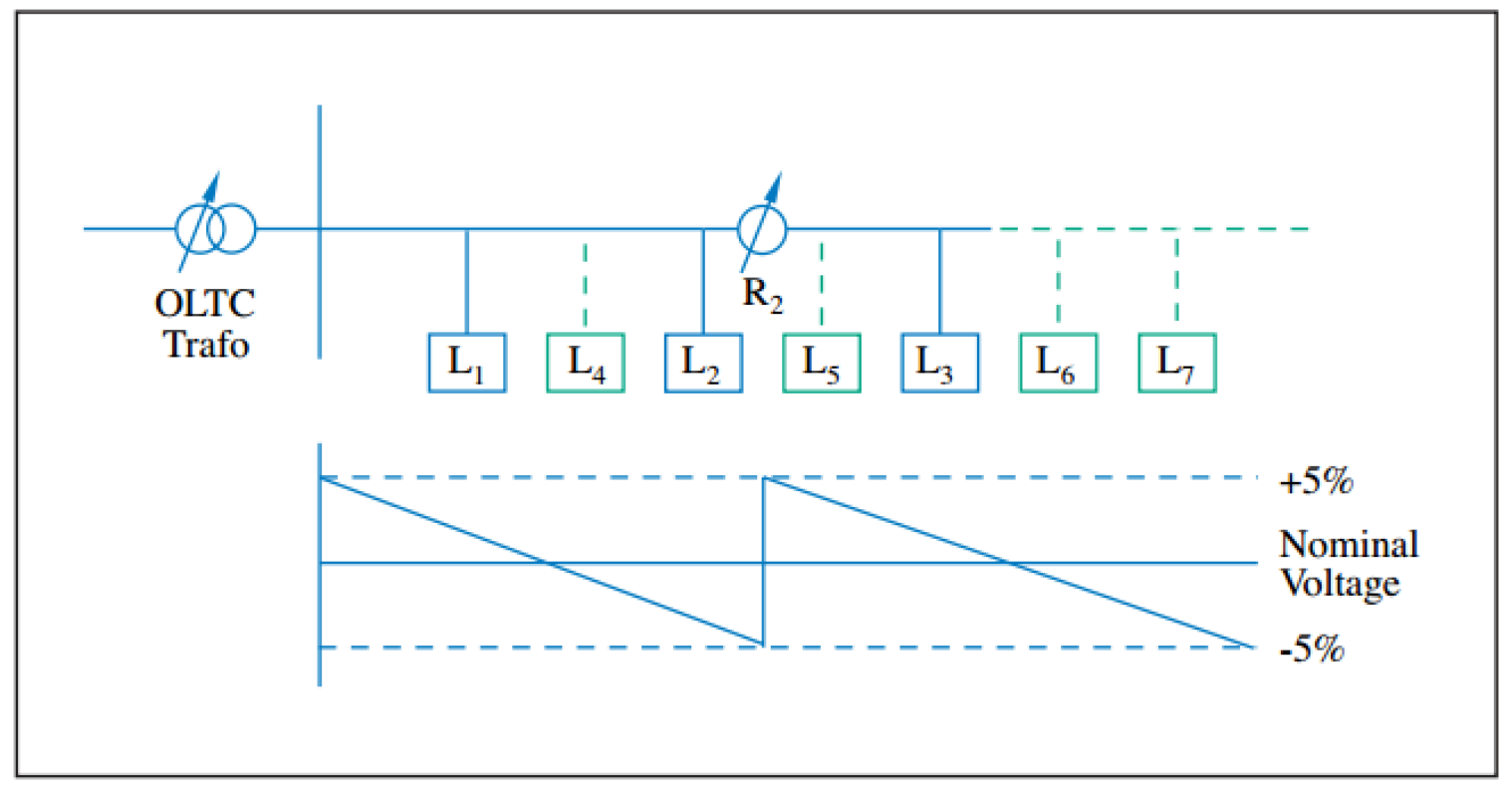

Figure 3-4: Adding a voltage regulator to improve voltage [20]

Typically radial circuits have predictable voltage profile - i.e. voltage downstream decreases - and this makes it easy to regulate the voltage. However, the addition of a PV system can cause a rise in the voltage near the point of interconnection; this makes it difficult to predict the voltage profile of the line, thus increasing the complexity of regulating the voltage. Based on the location of the voltage regulator and load on the circuit, the regulating device may experience reverse power flow (possibly several times a day), and the device settings need to be such that the voltage is properly controlled under reverse power conditions.

Voltage regulators are electromechanical devices. The variability introduced by PV systems increases the number of operations performed by the voltage regulators; in some cases the number of operations is up to an order of magnitude higher on a partially cloudy day as compared to a clear day. More frequent operations increases the maintenance cost over time [15]. In fact, many modern voltage regulator controllers calculate the service life remaining and the need for maintenance based on the number 
of tap changing occurrences [22]. There are several fixed and movable contacts in a voltage regulator. These contacts make and break connections while still supplying the load, and this causes arcing and contact erosion with each switching operation. Figure 3-5 illustrates the difference between a new set of movable contacts and one that has reached the end of service life.

New Contacts

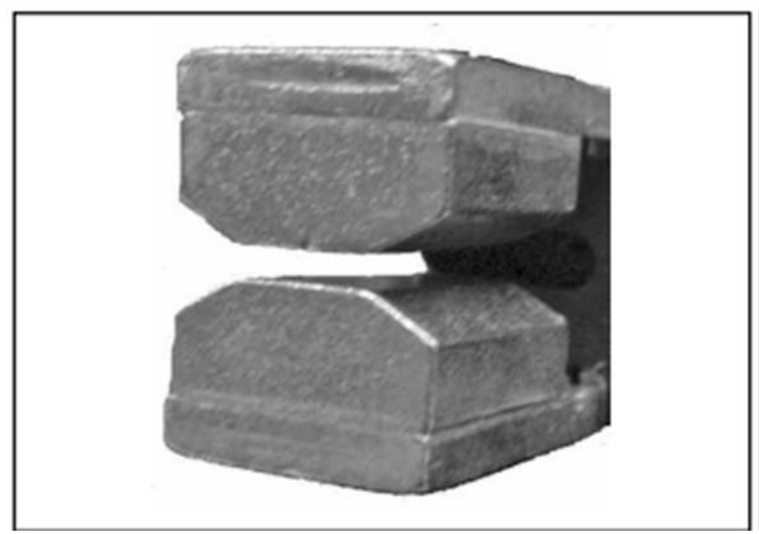

End of Contact Life Stage

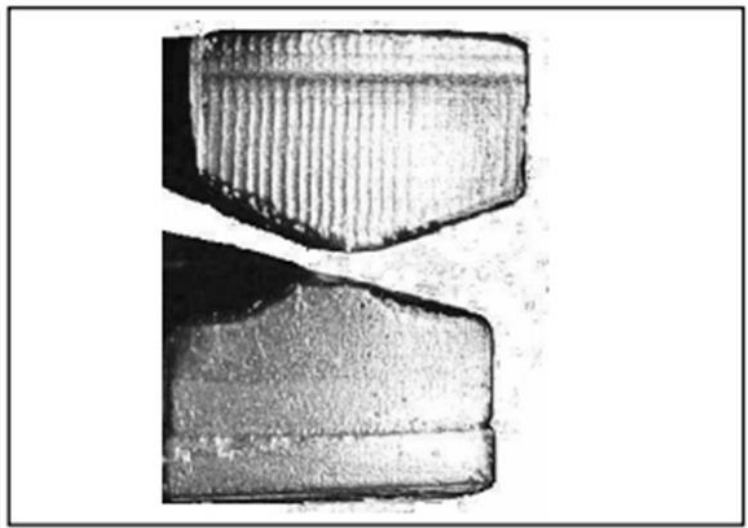

Figure 3-5: Voltage regulator movable tap-changer contacts [23]

In order to service voltage regulation devices, maintenance crews need to disconnect the device from the circuit. This is often done by bypassing the device, but for the duration that the device is out of service, it is possible for the voltage to remain out of allowable limits. However, in some cases it may not be possible to bypass the device. This would be the case when servicing the substation load tap changer; in this situation the circuit would need to be connected to another transformer, or in some cases the circuit would be blacked out for the length of the maintenance duration. 


\subsection{Existing Index}

In a study done by GE Energy, an index called "coefficient of increased switching duty" (CISD) is defined and used as a tool to compare the number of switching actions of a particular day to a base day [24]. The study created a simplified feeder model based on a $12.5 \mathrm{kV}$ radial distribution circuit. The model was simplified by reducing the number of nodes and aggregating loads, so that the simulation time and complexity would be reasonable, yet the model persevered enough detail to accurately depict the impact on the equipment. The circuit used in the study is shown in Figure 3-6. It is a radial circuit that splits up into two main laterals, and the ends of the laterals are connected to a breaker that is normally open. There is a substation load tap changing transformer between bus 102 and 103. There are six voltage regulators total on busses 202, 207, $209,304,306$, and 307 that can adjust the voltage by $+/-10 \%$ in 32 steps. All the regulators have a 60 second delay except the regulator on bus 307 that has a 75 second delay. The aggregated load as a percent of the total feeder load is shown in Table 3-1. The circuit also contains a 1.2MVAR fixed capacitor bank at bus 303, and a 1.2MVAR switched capacitor bank at bus 206 .

Table 3-1: Aggregated load at each bus

\begin{tabular}{|c|c|c|c|c|c|}
\hline Bus \# & Load (\%) & Bus \# & Load (\%) & Bus \# & Load (\%) \\
\hline 103 & 6.23 & 204 & 12.46 & 303 & 1.26 \\
\hline 104 & 8.72 & 205 & 3.54 & 304 & 0.12 \\
\hline 105 & 8.41 & 206 & 1.62 & 305 & 6.95 \\
\hline 106 & 8.45 & 207 & 1.00 & 306 & 3.43 \\
\hline 107 & 5.06 & 208 & 9.28 & 307 & 3.78 \\
\hline 201 & 4.05 & 209 & 3.12 & 308 & 2.29 \\
\hline 202 & 3.45 & 301 & 1.20 & 309 & 2.54 \\
\hline 203 & 0.00 & 302 & 1.35 & 310 & 1.68 \\
\hline
\end{tabular}



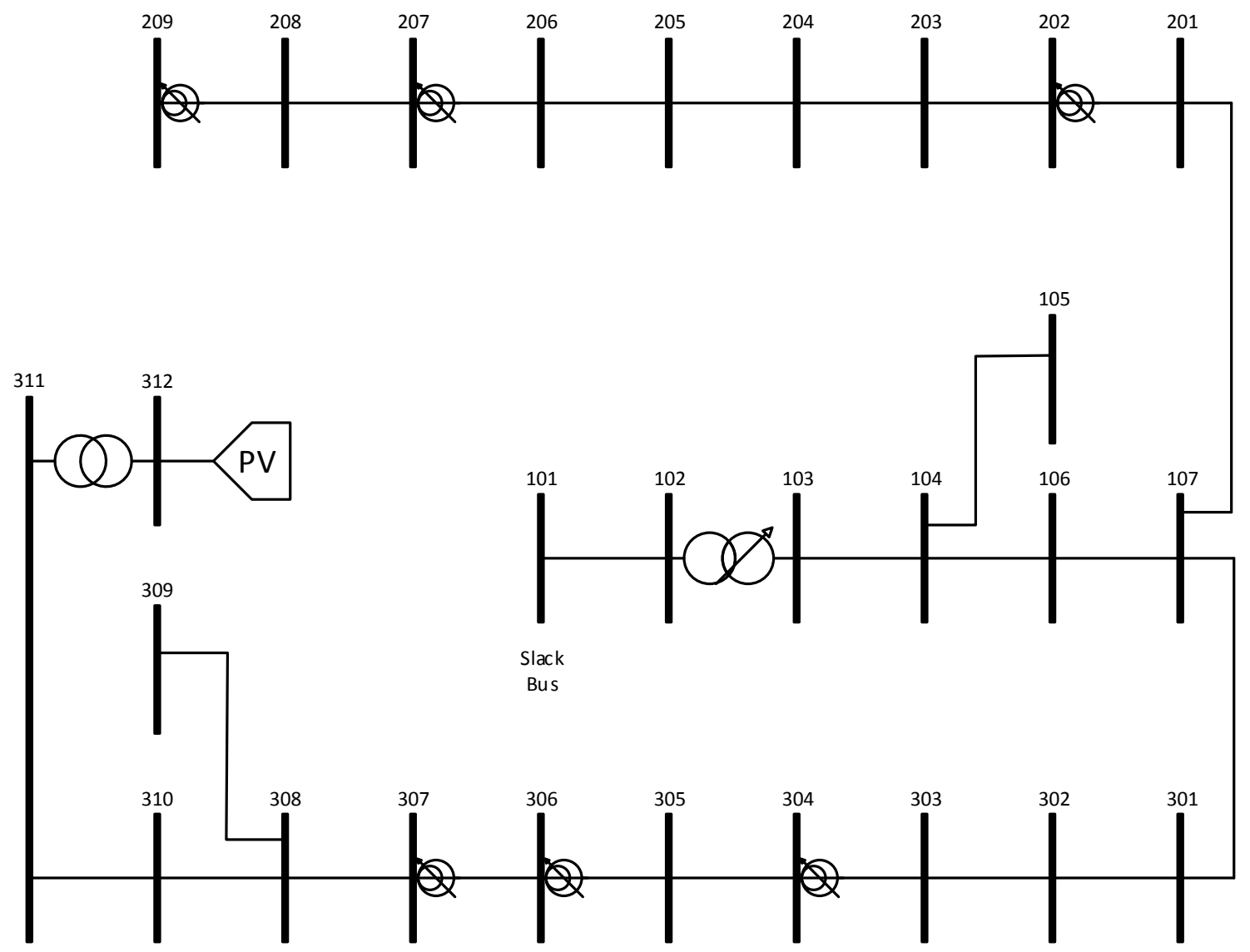

Figure 3-6: Radial circuit used in [24]

At the end of one of the laterals, there exists a 2MW PV generation facility; this is interconnected to bus 311 through a $480 \mathrm{~V} / 12.5 \mathrm{kV}$ step-up transformer as shown in Figure 3-6. To study the dynamic system, the feeder load profile and solar profile was created. A PV system model that would inject power into the circuit based on a user created solar profile is used in the simulation. The solar profile was created using data collected from GE's 700kW PV system located in Durham NC. The power data from a partially cloudy day and a clear day was scaled to $2 \mathrm{MW}$ and used for the study.

The study selected feeder load profiles for eight days representing a cross sample of various seasonal and weekly load profiles. These days were simulated along with the PV 
system connected to the grid and injecting a clear day power profile. The minimum feeder load day was selected to be the base day, and the other days were compared to this day as follows:

$$
C I S D=\frac{\sum \text { switching operations of all devices for a certain day }}{\sum \text { switching operations of all devices for the base day }}
$$

To simulate the effects of partially cloudy day, the same eight load profiles were used, however the power profile for the partially cloudy day was injected into the PV system model. The simulation resulted in an increase in the number of switching occurrences thus leading to a higher CISD value. Some of the results from the study are listed in Table 3-2.

Table 3-2: Number of switching operations of devices on a radial circuit

\begin{tabular}{|c|c|c|c|c|c|c|}
\cline { 2 - 7 } \multicolumn{1}{c|}{} & \multicolumn{2}{c|}{ Clear day } & \multicolumn{2}{c|}{ Partly cloudy day } & \multicolumn{2}{c|}{ Mitigation } \\
\hline $\begin{array}{c}\text { Device (bus } \\
\#)\end{array}$ & $\begin{array}{c}\text { Min load } \\
\text { used as } \\
\text { base }\end{array}$ & $\begin{array}{c}\text { Peak } \\
\text { load }\end{array}$ & Min load & $\begin{array}{c}\text { Fast Q } \\
\text { Peak } \\
\text { load } \\
\text { at } \\
311\end{array}$ & $\begin{array}{c}\text { 20kW/Min } \\
\text { ramp limit }\end{array}$ \\
\hline LTC (103) & 10 & 14 & 100 & 100 & 126 & 12 \\
\hline V.R. (307) & 15 & 25 & 141 & 153 & 57 & 20 \\
\hline V.R. (306) & 8 & 10 & 134 & 92 & 45 & 10 \\
\hline V.R. (304) & 5 & 12 & 35 & 46 & 68 & 5 \\
\hline V.R. (209) & 7 & 22 & 17 & 42 & 68 & 11 \\
\hline V.R. (207) & 8 & 30 & 20 & 46 & 56 & 9 \\
\hline V.R. (202) & 10 & 23 & 12 & 23 & 46 & 6 \\
\hline Sw. Cap. & & & & & & \\
(206) & 0 & 2 & 0 & 2 & 0 & 0 \\
\hline Total & 63 & 138 & 459 & 504 & 466 & 73 \\
\hline CISD & 1.00 & 2.19 & 7.29 & 8.00 & 7.40 & 1.16 \\
\hline
\end{tabular}


The study then investigate some mitigation options such as adjusting the delay settings on the voltage regulators, providing fast reactive power source, and limiting the PV system's ramp rates using energy storage. However, for the mitigation options, the simulation was done using only the load profile of the minimum load day (base day) and the cloudy day PV system profile. The simulation was performed several times while relocating the fast reactive power source to different busses, and the study determined the most ideal location for the fast reactive power source was at bus 311 . The results of this and of limiting the ramp rates to a maximum change of $20 \mathrm{~kW}$ per minute are also listed in Table 3-2.

The mitigation option that limits the ramp rates of the PV system's output power seems to be the best option, yet often times this option is not available to the utility since it requires modifying or controlling the customer's equipment and the need for onsite energy storage. This is a case where a change in policy that required the PV system operator to limit the ramp rates would be beneficial to the utility as well as other customers on the circuit. The study recommended the mitigation option of placing the fast reactive power source at bus 311 . However, notice that the CISD value for that option is similar to the value for a partially cloudy day with no mitigation implemented. This is one of the limitations of the CISD. In the next section, this and other weaknesses of using CISD as a metric for determining the impact on the regulation equipment will be examined, and the factors necessary for a more robust metric will be determined. 


\subsection{Index Considerations}

There is not much difference between the CISD values calculated for a partly cloudy day as compared to the fast $Q$ source mitigation day as shown in Table 3-2. However there is a difference in the switching occurrences for individual devices between the two simulated scenarios; the difference is that adding the fast reactive power source at bus 311 results in a reduction of switching for the devices close to the PV system, but increases switching for the other devices on the circuit. The study states that the " $Q$ based mitigation works by distributing the impact, and not by eliminating it" [24]. This implies the assumption that the importance and maintenance cost of all devices are equal, but this might not always be a valid assumption. As previously discussed, certain devices will affect a larger number of customers and will have higher maintenance cost than other devices. For example: line voltage regulators can easily be taken out of service for maintenance or replacement using the bypass switch, but a substation LTC transformer would require the load to be dropped or transferred in order to perform maintenance. Generally the devices closer to the substation will be more important than downstream devices. Thus a robust index should incorporate a weighting system for each device.

Another drawback to using the CISD is that there is no intuitive feel to the numbers, and it is difficult to determine the additional wear cause based on the CISD value. The metric compares the effects of partially cloudy day to a clear day. Depending on the location, such a comparison might provide very little useful information; for instance, if the PV system is located in a desert environment where partially cloudy days are a rare occurrence, then even though the CISD may have a high value, the annual impact to the voltage regulation equipment would be close to the impact of a clear day. In order to make the index universally applicable and more intuitive, two key concerns must be 
addressed. Firstly the index must integrate the annual weather data for the location of the PV system i.e. the ratio of partially cloudy to clear days in a year ${ }^{2}$. Secondly the base case must be carefully selected to provide a valid comparison of the impact. The later can be better understood by examining the following scenarios. Suppose the purpose of the study is to determine the impact of adding a PV system to the circuit. Here the base case would be behavior of the circuit without the PV system connected, and that would be compared to the annual (i.e. consider effects clear days and partially cloudy days) behavior of the circuit with the addition of the PV system to the circuit. On the other hand, if the purpose of the study is to determine the impact of a particular mitigation option, say installing a fast reactive power source, then the base case should be the annual behavior of the circuit with the PV system but without the mitigation option. This base case should then be compared to the annual behavior of the circuit with both, the PV system and the fast reactive power source, connected. Selecting a proper base will allow to study the effects of mitigation options involving the addition of a voltage regulation equipment to the circuit, whereas such an option could distort the CISD value.

Lastly it is important to keep in mind the purpose of voltage regulating equipment - to supply the customer power while retaining the voltage within allowable limits. It is possible to reduce the wear on the devices by increasing the bandwidths and time delays, but doing so can cause the magnitude and time span of voltage fluctuations to also increase, so there is a trade-off between the customers' voltage quality and equipment wear. This is where the experience and judgment of the engineer performing the study comes into play; the engineer needs to determine the "acceptable" trade-off. The term acceptable is qualitative and is dependent on the situation. It might be

\footnotetext{
${ }^{2}$ This ignores the effect of days that are completely overcast. The impact of overcast days would fall between having no PV system on the circuit to the effect of a smaller system on a clear day. The accuracy of the impact study may be increased by accounting overcast days; however, slight improvement (if any) of accuracy does not justify the resultant significant increase in complexity.
} 
acceptable to relax the bandwidth and increase the time delay a bit for devices serving a residential load to extend the devices' service life, but it might be unacceptable to do the same for devices serving a hospital. Therefore it is important to examine the voltage quality side-by-side with the impact metric. In summary, to develop an index the following parameters must be considered:

a. A weighting factor for individual devices

b. The average number of partially cloudy days in a year

c. The trade-off between voltage quality and equipment wear

In the next section a metric will be developed that can be used to determine the impact of the addition of a PV system or mitigation option to the voltage regulation equipment on the distribution circuit.

\subsection{The Index}

The maintenance cost and service life of a regulating device is proportional to the number of switching operations performed by the device [15] [22]. An increase in switching operation leads to an increase in maintenance cost and a reduction of service life. Based on that, the key data for this metric is the number of switching operation performed by each device under different conditions such as without a PV system connected, with a PV system on a clear day, with a PV system on a partially cloudy day, etc. The conditions simulated will depend on the purpose of the impact study. Using a feeder load profile that represents the average day may seem intuitive; however it would be better to use a load profile that is representative of a low feeder load day. When the feeder load is lower, the variations of the PV system appear further into the system thus 
affecting more devices. Therefore using a feeder load profile closer to the lower end of the spectrum will provide the impact for a worst case scenario.

The following index given by equations (3-2) to (3-4) is describing the impact of the PV system on the circuit, so the base case (denominator of the equation (3-2)) here is the behavior of the distribution circuit without the PV system connected. However, the same method can be used to develop an index to study the impact of various mitigation options. For that scenario the base case would be the annual behavior of the circuit with the PV system connected; this would be the numerator of the equation (3-2).

\section{Device Cost Factor}

$$
D C F=\frac{\left(S_{C l r} * D_{C l r}\right)+\left(S_{P C} * D_{P C}\right)}{S_{A v g} * 365}
$$

where:

- $D C F$ : is the device cost factor. This value indicates how much higher $(D C F>1)$ or lower $(D C F<1)$ the service cost of a particular device in the circuit will be after the addition of the PV system. Alternatively, $\frac{1}{D C F}$ can provide insight into how the service life of the device will be affected.

- $S_{C l r}$ : is the number of switching actions of the device on an average clear day with the PV system in the circuit.

- $\quad D_{C l r}$ : is the number of clear days in a year at PV site. A simplifying assumption here is that completely cloudy days (overcast days) have a similar impact as clear days, and those days are also accounted for here. 
- $S_{P C}$ : is the number of switching actions of the device on an average partially cloudy day with the PV system in the circuit.

- $D_{P C}$ : is the number of partially cloudy days in a year, $D_{P C}=365-D_{C l r}$.

- $S_{A v g}$ : is the number of switching actions of the device on an average day without the PV system in the circuit.

\section{Device's New Operating Cost}

$$
N C=O C * D C F
$$

where:

- $N C$ : is the annual operational cost of the device after the addition of the PV system to the circuit.

- $O C$ : is the estimated annual operational cost of the device before adding the PV system to the circuit. The engineer performing the study selects a value that best represents the device. This is the device weighting factor. The values for the operating cost can be actual dollar amounts, or can be normalized against a particular base device in the circuit.

- $\quad D C F$ : is the device cost factor calculated in equation (3-2).

Circuit Impact Index

$$
C I I=\frac{\sum N C \text { for all devices }}{\sum O C \text { for all devices }}
$$


The circuit impact index value provides insight into how much the operational cost for all voltage regulating devices in the circuit will increase $(C I I>1)$ or decrease $(C I I<1)$ by; in other words, it represents how much more/less will be spent on existing voltage regulating equipment every year. An example of using this index will be provided in Chapter 5.

\section{Voltage Quality}

The engineer performing the impact study also needs to evaluate the voltage quality to ensure it is acceptable. There already exists different voltage quality metrics, and most utilities have adopted a certain voltage quality metric for use by the engineers. It would be beneficial for the engineer performing the study to use the metric already used by the utility or something similar, and to gather the data necessary for the voltage quality metric while simulating various scenarios. Doing so would allow the engineer and his/her colleagues to better understand the result, since they would be accustomed to the metric. One such metric that is used by several utilities is the System Average RMS Variation Frequency Index $\left(\mathrm{SARFI}_{\mathrm{X}}\right)$. For the purpose of this thesis, the voltage quality will be examined by the minimum and maximum voltage levels, and the time duration that the voltage is above or below specified limits. 


\section{PSCAD Modeling}

The correctness of the impact study will depend on the accuracy of the models used to represent the PV system, distribution circuit, regulating devices etc. and the accuracy of the solar and feeder load profiles. Often times the goal is to create models that represent reality as best as possible, yet keeping the complexity level low enough to allow simulation to be completed within a reasonable time. Another important consideration is selecting the proper simulation software that is powerful and versatile.

The software selected to simulate the test cases in this thesis is Power Systems Computer Aided Design (PSCAD). PSCAD is a simulation software that allows the seamless integration of power electronics and power systems to study power system transients. This chapter will describe the models created for the study, and in doing so, some of the features of PSCAD will be presented.

\subsection{System Overview}

The study performed in this thesis is an example of a study that examines the impact of adding a 2MW PV system to a distribution circuit. Figure 4-1 provides an overview of the system that will be created in PSCAD to perform this study. The orange ovals represent system inputs, whereas the green ovals are system outputs; these inputs / outputs are read from / written to files as will be discussed later. Depending on the computer speed, the amount of data, and the complexity of the models, the simulation can be time intensive, so in order to automate the process of simulating the various scenarios, the necessary control logic was created to run the simulation multiple times while providing the PV system with a different input set for each run. 


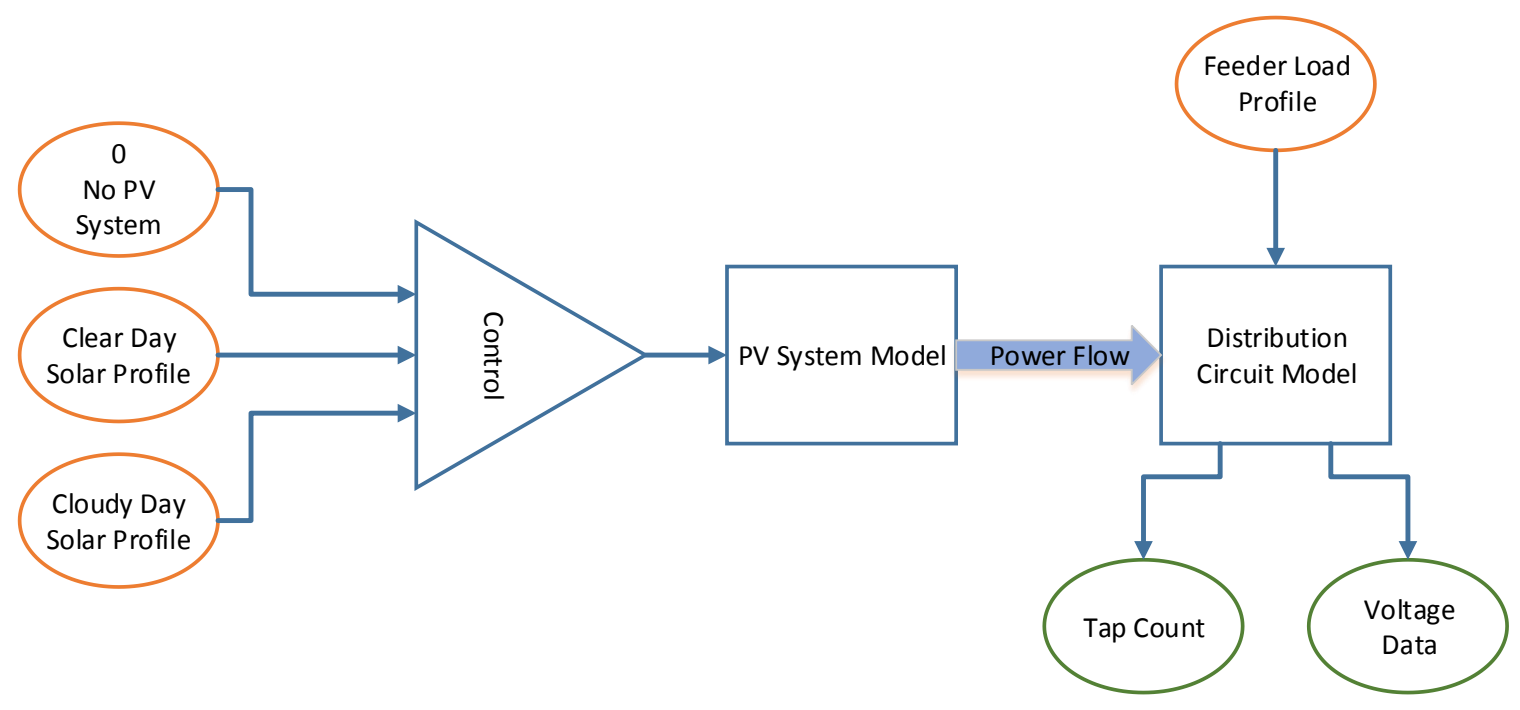

Figure 4-1: Impact study system overview

The high-level view of the system as implemented in PSCAD can be seen in Figure 4-2. The Multiple Run module ${ }^{3}$ in the top left corner allows users to automate the process of simulating different test cases. This module can output up to six variables that change values for each run as defined by the user. Here the module outputs just one variable "V1" which is then labeled as "Ctrl". The signal "Ctrl" then processed using a level comparator to create a secondary signal "PVctrl", and the two signals are used to select the data that is passed into the PV system for each run. The level comparator is set to output a 1 if the input is greater than 1.5 and 0 otherwise. For the first run, "Ctrl" is given a value of 3 which makes the value of "PVctrl" to be 1 , thus passing zero for the radiation and temperature values into the PV system module. Due to this, the PV system produces no power, and this represents the simulation for the case where the PV system is not connected to the distribution system. Table 4-1 presents a summary of how the data selecting controller operates.

\footnotetext{
${ }^{3}$ Module and component are interchangeable terminology.
} 

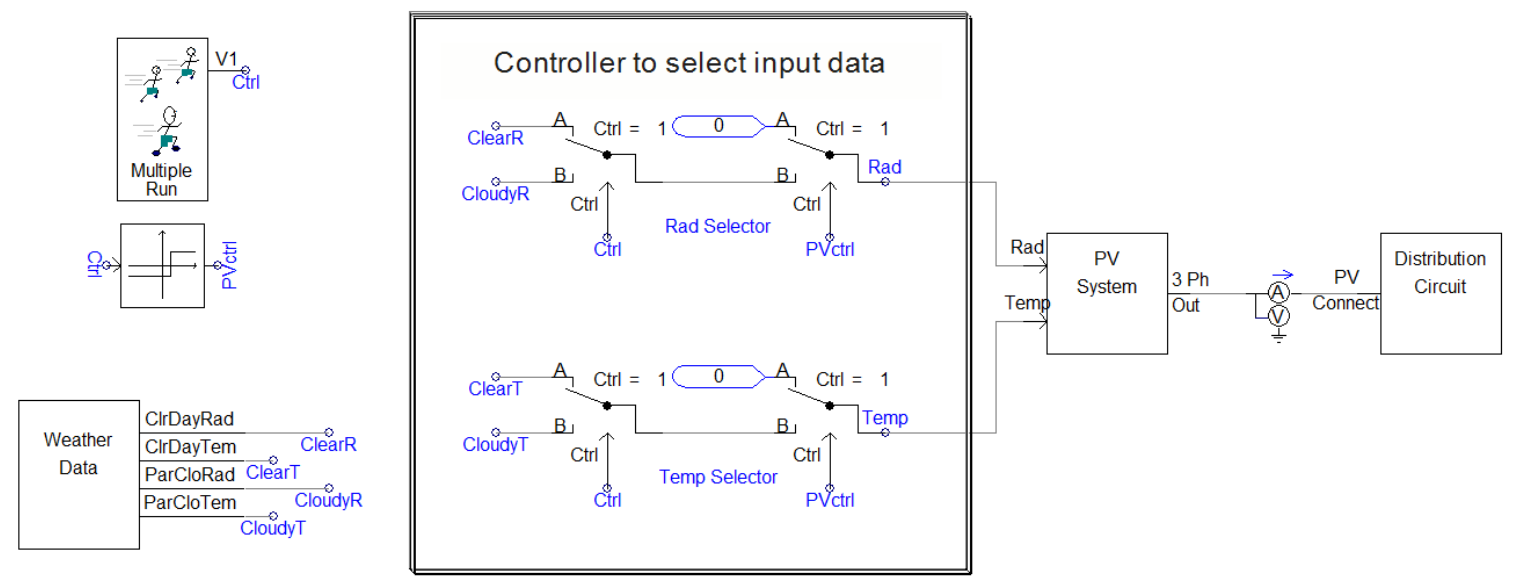

Figure 4-2: System implemented in PSCAD

Table 4-1: Functional logic for data selection

\begin{tabular}{|c|cccc|}
\hline Ctrl & PVctrl & $\begin{array}{c}\text { Left } \\
\text { switch }\end{array}$ & $\begin{array}{c}\text { Right } \\
\text { switch }\end{array}$ & $\begin{array}{c}\text { Data to PV } \\
\text { system }\end{array}$ \\
\hline 3 & 1 & n/a & A & 0 (no PV system) \\
\hline 1 & 0 & A & B & Clear day \\
\hline 0 & 0 & B & B & $\begin{array}{c}\text { Partially cloudy } \\
\text { day }\end{array}$ \\
\hline
\end{tabular}

A convenient feature of PSCAD is the ability to create modules. The modules labeled Weather Data, PV System, and Distribution Circuit are user created modules. Creating modules allow for better organization which can make the project visually appealing and easier to troubleshoot; another benefit is that modules can be reused multiple times in the project or even imported for use in other projects. Figure 4-3 shows the internal structure of the Weather Data module. This module performs a simple function of reading the data from a file (Input.txt), and passing the data to the output ports of the module. The component above the Input.txt file is the File Read component, and it can read and output up to eleven scalar data values written as columns in the file. 
It can output the values at user defined time intervals which would be entered in the first column (so only a maximum of ten data columns would remain), or the user can set a sampling frequency and use all eleven columns for data. For this simulation a known sampling frequency of $10 \mathrm{~Hz}$ was selected. The data used in the Input.txt file can be found in Appendix A: Solar Profile Data. The Weather Data module discussed here is a rather trivial module, but as we will see in the next sections, modules can get quite complex and can contain several sub modules.

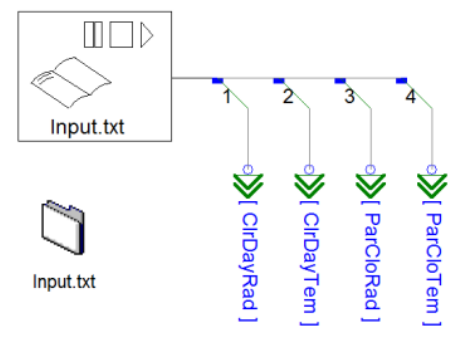

Figure 4-3: Contents of the Weather Data module

\subsection{Modeling the PV System}

PSCAD is a powerful tool capable of integrating power electronics into power systems. It is possible to create and simulate an actual three phase inverter using power electronics devices. However, the impact study would ideally simulate the test cases for at least eight to twelve hour periods to capture the effect of the entire range of irradiance from sunrise to sunset. In this situation, the time and computing resources needed would make it impractical to use a complex model using power electronics, as the simulation time step would need to be smaller than the switching frequencies which are in the kiloHertz range. To resolve this problem, the complexity of the PV system model must be reduced, while ensuring the behavior and effect of the model to the distribution system is an accurate representation of real systems. 


\section{PV System Model}

Delays the PV system for $0.05 \mathrm{sec}$ in order to prevent transients.

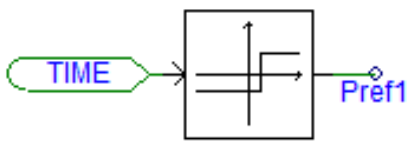

The interconnect voltage is used to generate reference signal for the current sources.

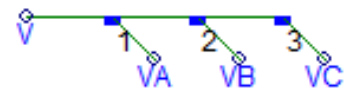

The voltage and PV panel power reference are processed to output the proper current waveforms.

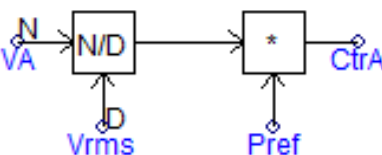

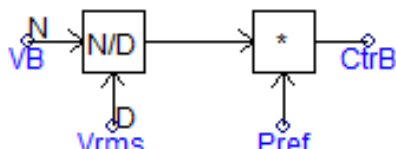

vrms Pref

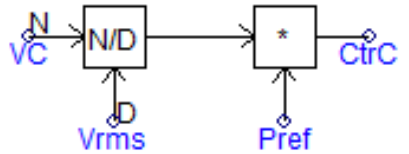

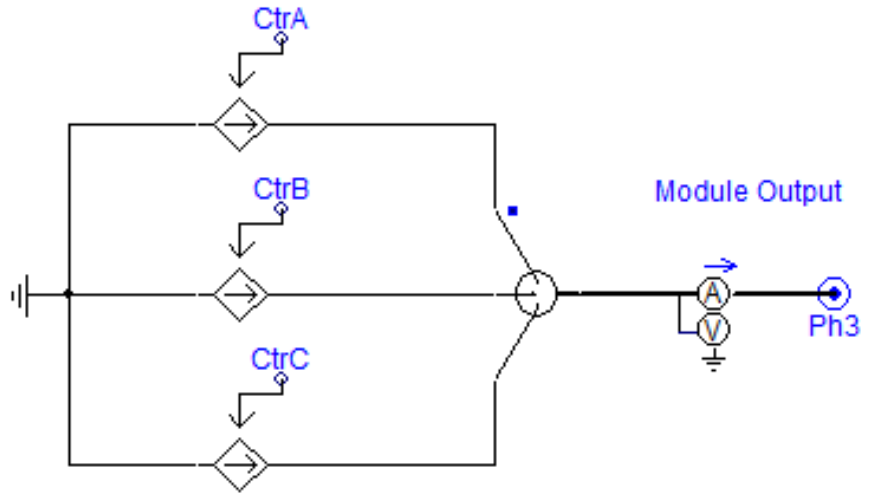

PV panel is used to recreate the behavior of the PV array as the solar radiation and temperature changes. The output is then scaled up to be used as a reference for the current source.

Module Inputs
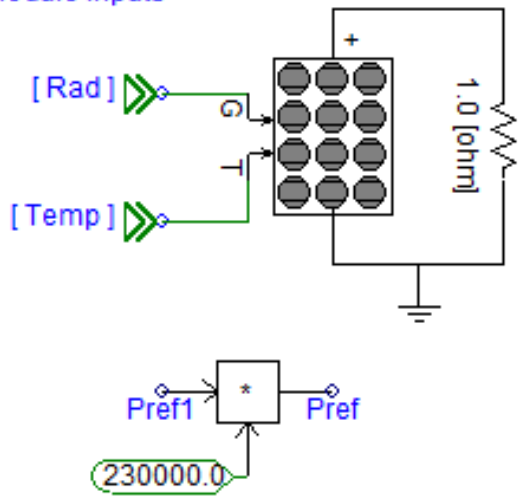

Figure 4-4: Contents of the PV System module

The first step in forming a simplified model is to investigate the behavior of the real system and examine what basic components can be used to recreate the behavior. In the case of the PV systems, we can use dependent current sources to inject current at the point of interconnection, and this is because PV inverters that are synchronized with the grid voltage act as nearly ideal current sources [11]. Under existing standards and 
policies, the PV system operates with a fixed power factor at (or very close to) unity, so the dependent current sources need to inject current that is in phase with the voltage at the interconnection point.

The PV system model developed in PSCAD for this study is shown in Figure 4-4. The main aspect of the PV system is represented by the three dependent current sources connected to the three phase output port in the upper right area; everything else represents the processing required to properly control the dependent current source based on the radiation and temperature data received. The inputs to the module ("Rad" and “Temp") are fed into PSCAD's Photovoltaic Source component. The purpose of this is to use the PV panel to translate the solar profile data to power output data i.e. the PV panel outputs power corresponding to the solar profile and this output power is used to determine the output of the current sources. The power output of the PV panel is labeled "Ppanel", and is fed to the signal "Pref1" after a time delay of 0.05 seconds as shown in Figure 4-5. The signal "Pref1" is suitably scaled and multiplied by the appropriate phase voltage reference signal to produce a signal that can control the current source connected to that particular phase. The voltage reference signal is produced by measuring the instantaneous voltage at the interconnection point (the module's output labeled "Ph3") and normalizing this with the rms voltage; doing so, reduces the amount of output power variation of the PV system model caused by variation of the interconnection voltage. 


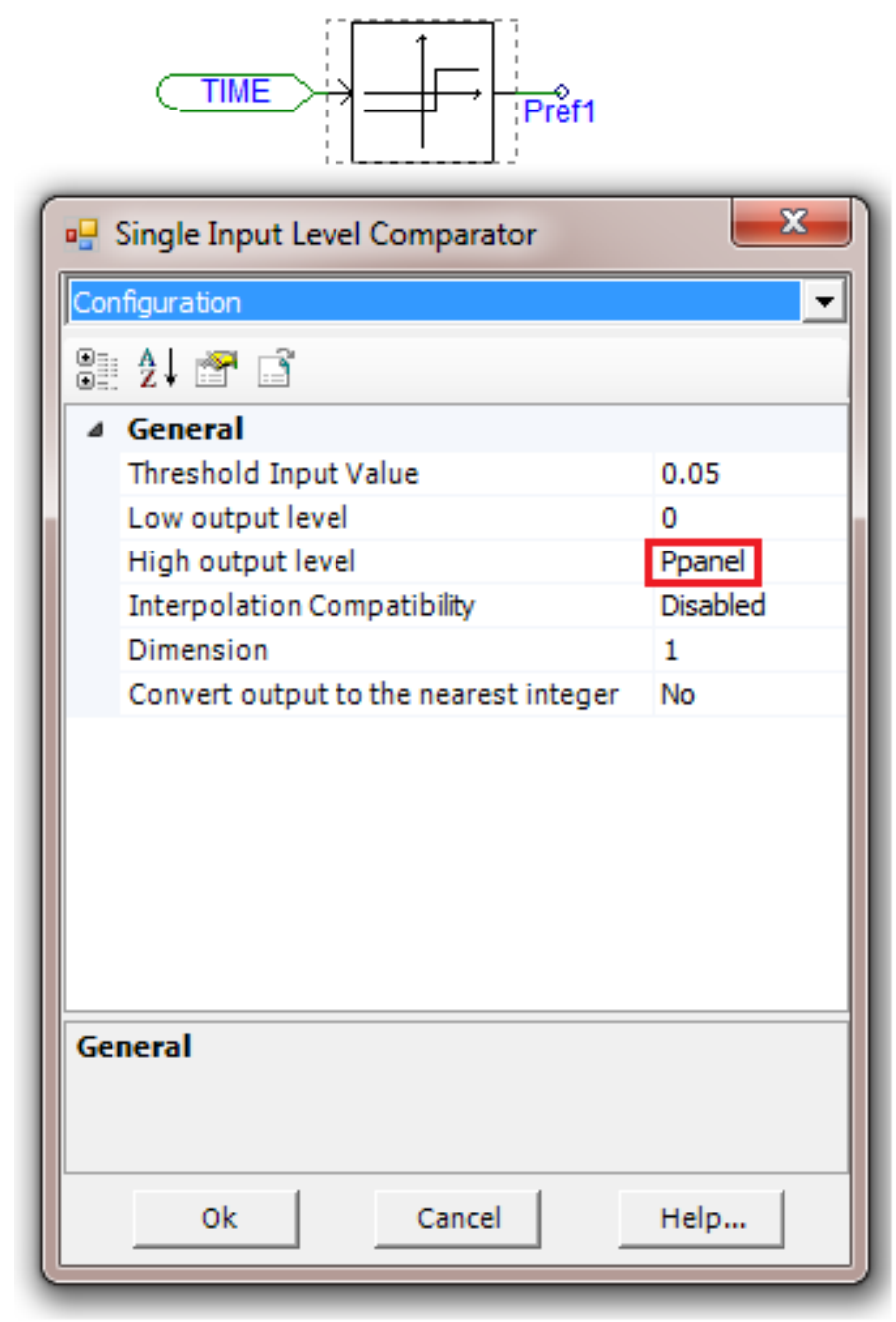

Figure 4-5: Producing a time delay before the PV system model generates power

Using a Windows XP computer a few years old, the time to simulate the complete system described in this chapter was about four times the simulation period i.e. it took almost four minutes to simulate 1 minute of the circuit behavior. For the purpose of this study, the given time constraint was reasonable since each scenario was only simulated for a period of 60 seconds as will be described in the next chapter. However, if this time constraint is unreasonable when performing an actual impact study - due to the need of simulating for much longer time periods - then it may be necessary to further simplify the models used. It is possible to simplify the PV system model by eliminating the use of the 
PV panel in the model, and feeding the module the desired output power that is precomputed using the solar profile data.

\subsection{Modeling the Distribution Circuit}

The distribution circuit used for the impact study is a recreation of the circuit used in [24] as was described in Section 3.3 with some slight modifications. The most notable modifications are the elimination of the switched capacitor bank, and the elimination of the step-up transformer used to connect the PV system to the distribution circuit. The internal structure of the distribution circuit module is shown in Figure 4-6. The distribution circuit occupies the bulk of the space, and the other components are needed for providing the feeder load profile, automating the various runs, plotting graphs, and saving the necessary data to output files.

The PV system is connected to busses 209 and 310 via breakers. The logics that control the breakers, using the signals "BRK1" and "BRK2", appear in the upper left corner. The breakers are used to automate the simulation of multiple test cases as will be described in the next chapter. Below the breaker control logic there is the File Read component that reads the feeder load profile data (Load_Data.txt) at a sampling rate of $1 \mathrm{~Hz}$ and outputs the values to signals "R1" through "R11". The data used in the Load_Data.txt file can be found in Appendix B: Feeder Load Profile Data. The signals "R1" to "R11" are used to control variable resistors connected on busses $104,107,302$, $304,306,307,310,202,204,206$, and 208 respectively; this is better shown on a larger view of the circuit given in Figure 4-7. 

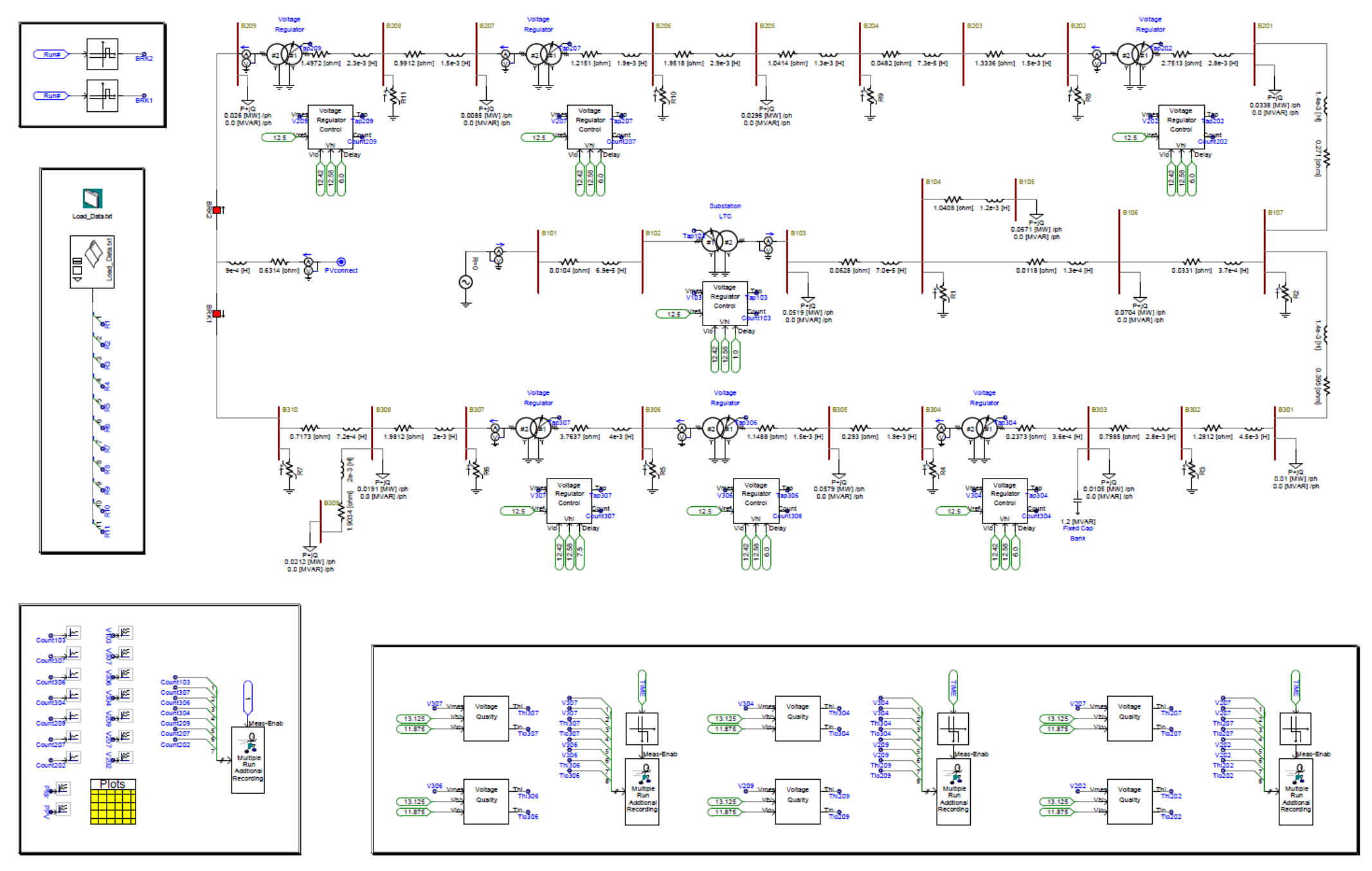

Figure 4-6: Contents of the Distribution Circuit module 


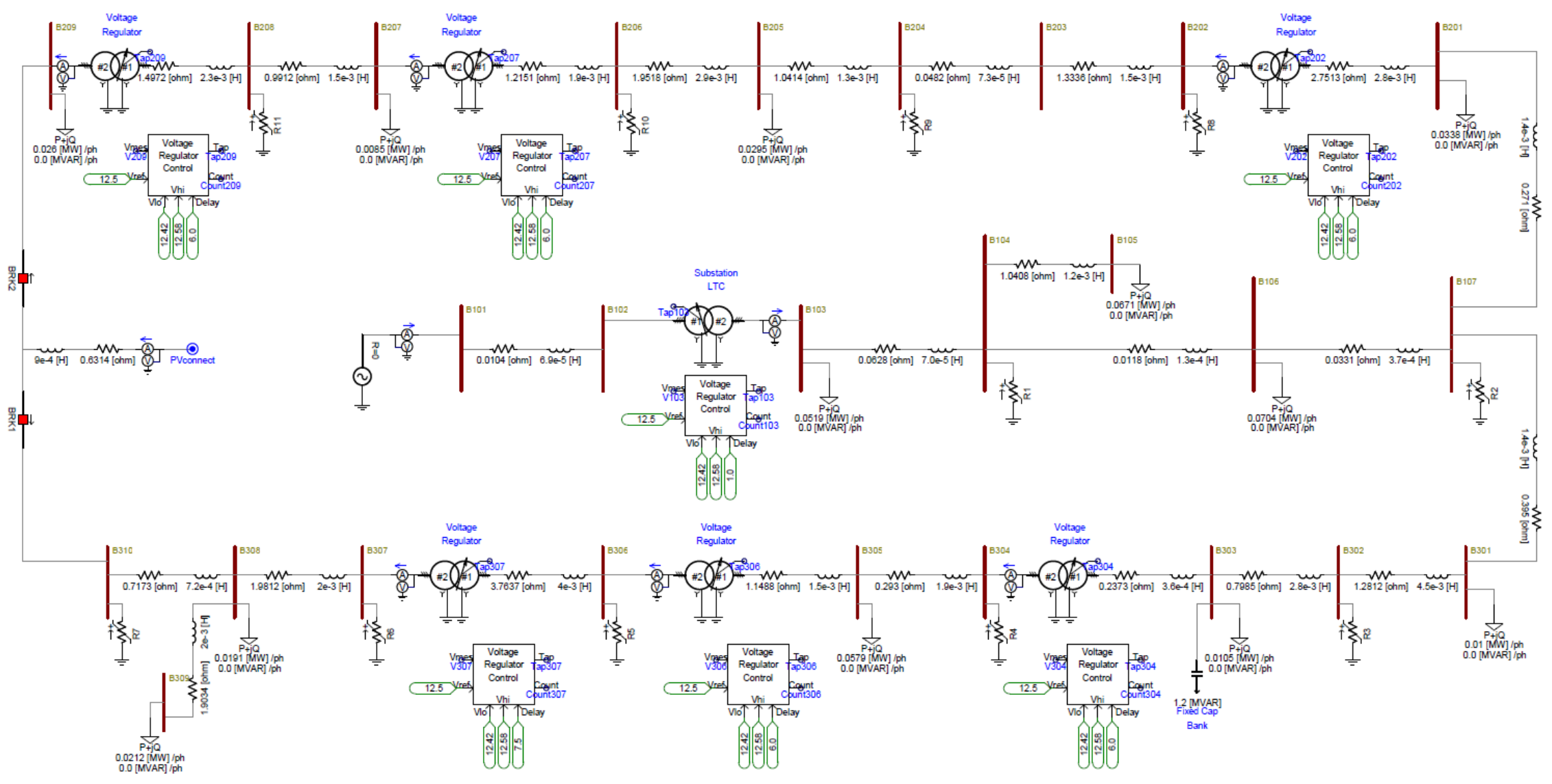

Figure 4-7: Radial circuit in PSCAD 


\subsection{Modeling the Voltage Regulator}

Transformer models in PSCAD allow for a tap changing winding, and this is what was used to create the behavior of the voltage regulator. However, there is no pre-made component that can be used to generate the signal to control the tap settings. To accomplish this, the Voltage Regulator Controller module was created. These modules are placed below each voltage regulating transformer as seen in Figure 4-7. Other than the substation LTC, the voltage regulator transformers have a 1:1 turns ratio. The adjustable winding is on the downstream (secondary) side of the transformer, and this is the point where the voltage is measured and used as the "Vmes" of the Voltage Regulator Controller module. The reference voltage, time delay, and the high and low voltage limits are parameters that are specified by the user using the Real Constant component. The output of the Voltage Regulator Controller module is a tap position and the number of times the taps have been changed during a particular simulation run.

The logic used for the Voltage Regulator Controller module is shown in Figure 4-8. The measured voltage is compared to the low and high voltage limits, and if the measured voltage is either below or above the limits and remains out of limits for the duration of "Delay" seconds, then the corresponding "lo" or "hi" signal is assigned a value of 1 . When the value of either "lo" or "hi" is 1 , then the integrator begins to integrate the "Error" signal and adjusts the "Tap" accordingly. Once the measured voltage is within limits again, the values of "lo" / "hi" is once again 0 , and the integrator is reset. The Sample and Hold component is used to ensure that "Tap" is held at the proper value and does not change when the integrator is reset. The internal upper and lower limits of the integrator are set for 1.1 and 0.9 respectively, as this represents the $+/-10 \%$ capability of voltage regulators. The schematic at the very bottom of Figure 4-8 is the logic that keeps track of the number of tap changing (switching) occurrences. For 
any change in the "lo" or "hi" signals, the sequential number generator component increments by one; since the signals change twice ( 0 to 1 and 1 to 0 ) for each tap change occurrence, the output is incremented by 0.5 for each occurrence and then assigned to "Count".

The voltage regulators being modeled here are based on $+/-10 \% 32$ step regulators. This means that the regulator can control the voltage by either raising or lowering the voltage by up to $10 \%$ in increments of approximately $5 / 8 \%(0.625 \%)$ steps for a total of 32 steps. To represent this, the minimum and maximum voltage threshold values for the regulator controller are as follows:

$$
\begin{gathered}
\text { Vlo }=12.5 *(1-0.00625)=12.42(\mathrm{kV}) \\
\text { Vhi }=12.5 * 1.00625=12.58(\mathrm{kV})
\end{gathered}
$$




\section{Voltage Regulator Contorller}
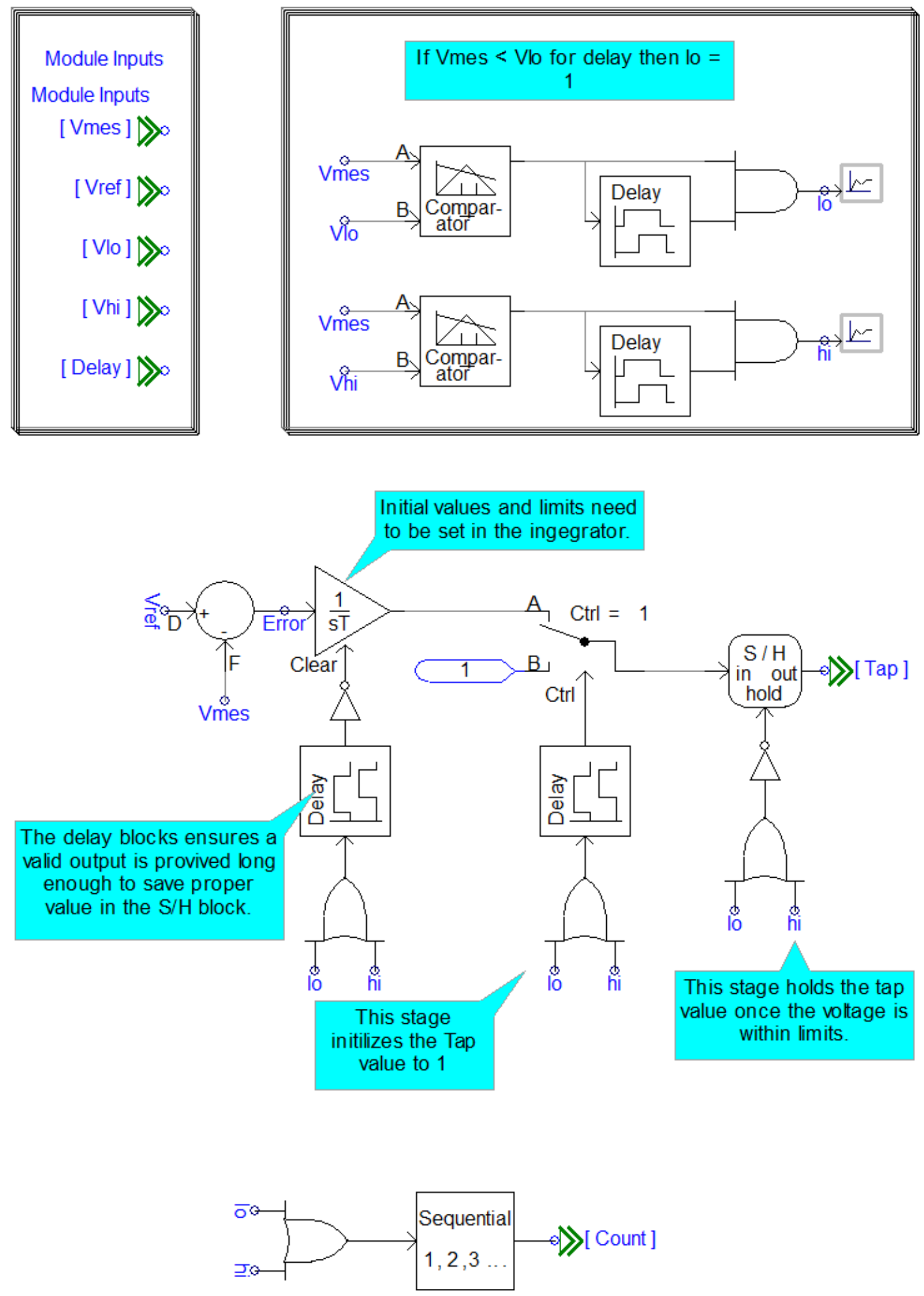

Figure 4-8: Contents of the Voltage Regulator Controller module 


\subsection{Voltage Quality and Data Collection}

For the purpose of this study, the voltage quality is determined by examining the maximum and minimum voltage level and the amount of time that the voltage is out of certain set limits. The Voltage Quality module was created to measure the time that the voltage at a particular node is above and below preset limits. The schematic for time module is shown in Figure 4-9. It is a simple logic system that feeds the integrator a value of 1 when the measured voltage is above / below the limit, and since the integrator's time constant is set to 1 second, it outputs the time (in seconds) that the measured voltage was higher / lower than the limit. A time delay of 8 seconds is introduced to allow each regulator to initially change the tap once if needed.

\section{Voltage Quality}

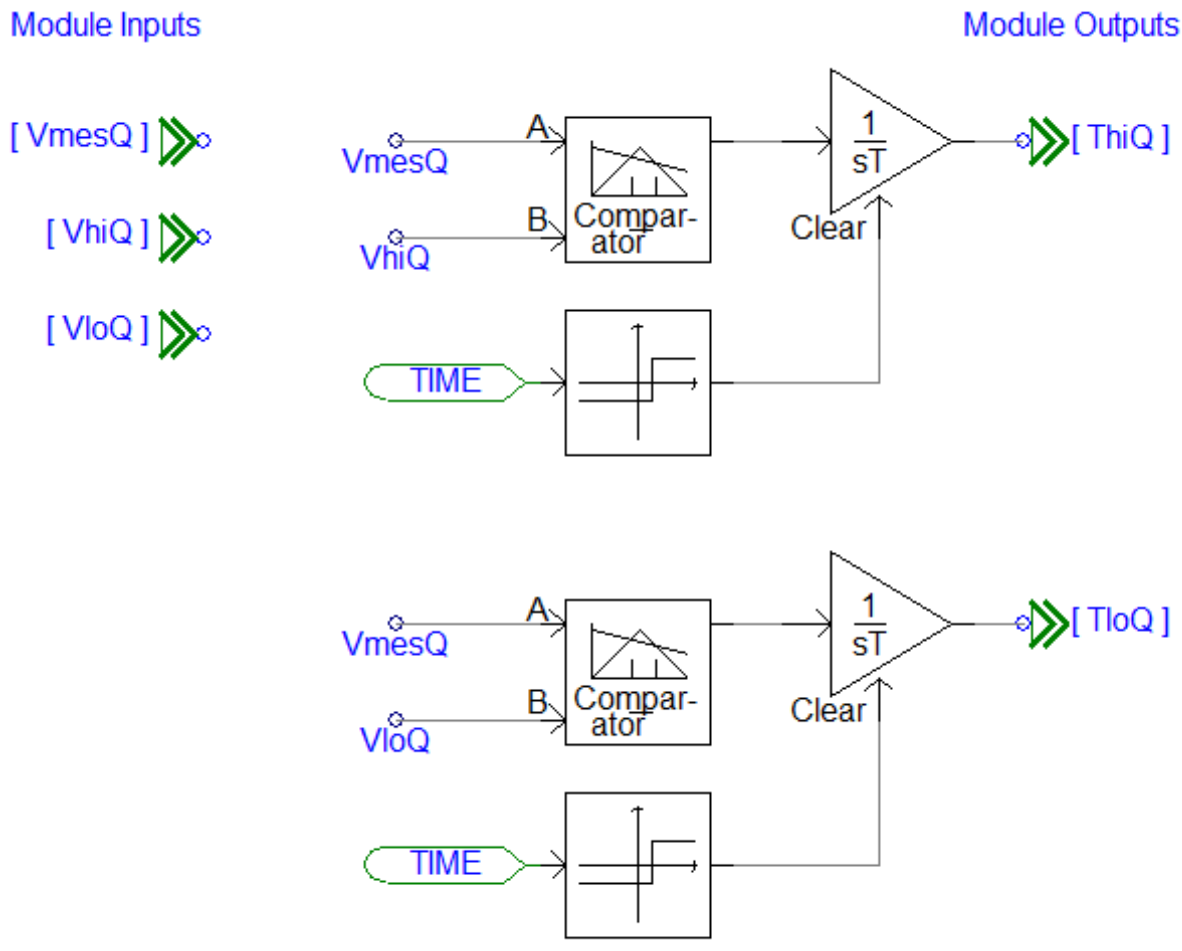

Figure 4-9: Contents of the Voltage Quality module 
In order to measure the maximum and minimum value of the voltage at a particular node during the simulation run, the Multiple Run Additional Recording component is used. These components store the data of each run in output files. A time delay of 8 seconds is introduced before measuring the maximum / minimum; this is to allow all the voltage regulators to adjust taps at least once if needed thus the data does not include the transients caused by PSCAD initializing the system. The components used to measure and record the voltage quality data for the voltage at bus 306 and 307 is shown in Figure 4-10. The tolerance limit is set to $+/-5 \%$; for instance, "Thi307" is the time that the voltage at bus 307 is above $13.125 \mathrm{kV}$, and "Tlo307" is the time the voltage is under $11.875 \mathrm{kV}$.
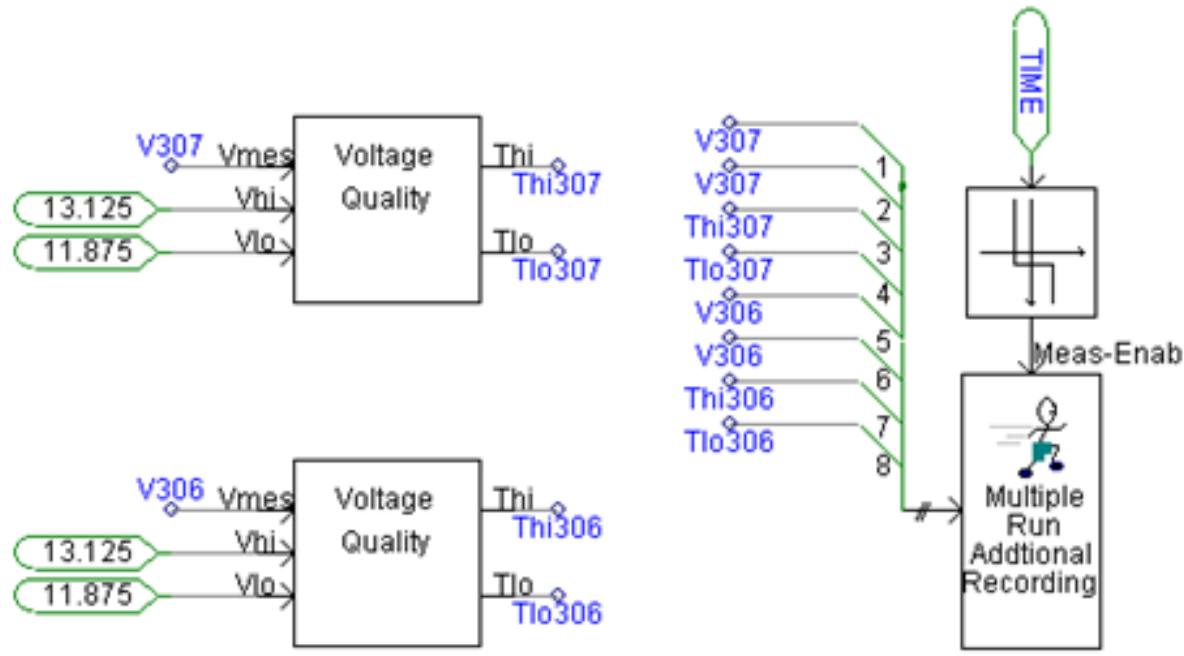

Figure 4-10: Measuring and saving the voltage quality data 


\section{Test Cases and Results}

This chapter will present an example of conducting an impact study. The goal of the study is to determine the optimum placement of the PV system. The circuit studied is the circuit being discussed thus far. The circuit is such that bus 209 and bus 310 are connected together with a breaker that is normally open. If the breaker is closed then the two laterals are combined and the circuit is partially radial with a loop circuit at the end. The PV system is planned to be located at the end of the circuit in close vicinity of bus 209 and 310. This allows for the following three interconnecting options: 1) connect to bus 310,2$)$ connect to both bus 310 and 209 by closing the breaker and operating the end of the circuit as a loop, or 3) connect to bus 209. The economic impact of all three options would be quite similar, thus the only considerations should be equipment impact and voltage quality.

\subsection{Setup}

When the study involves exploring different options, using the multiple run feature of PSCAD can be very beneficial because it automates the process and allows the user to perform other tasks while the various scenarios are being simulated. The set up for being able to use multiple run is shown Figure $5-1$, where the PV system can connect to either (or both) bus 209 or 310 with the use of two breakers. To simulate the three options, seven runs were set up; the first run is simulated with no PV on the circuit to establish the base case, and the other six runs simulate the clear day and partially cloudy day behavior for each of the three options. Details of the condition simulated in each run and the breaker states are provided in Table 5-1. 
Table 5-1: Conditions simulated during each run

\begin{tabular}{|c|c|c|c|c|}
\hline Run \# & Condition & $\begin{array}{c}\text { Connected } \\
\text { to } \\
\end{array}$ & BRK1 & BRK2 \\
\hline 1 & "No PV & n/a (B310) & On (0) & $\overline{O \text { Off (1) }}$ \\
\hline 2 & Clear day & \multirow{2}{*}{ B310 } & \multirow{2}{*}{ On } & \multirow{2}{*}{ Off } \\
\hline 3 & Cloudy day & & & \\
\hline 4 & Clear day & \multirow{2}{*}{$\begin{array}{c}\text { B310 \& } \\
\text { B209 }\end{array}$} & \multirow{2}{*}{ On } & \multirow{2}{*}{ On } \\
\hline 5 & Cloudy day & & & \\
\hline 6 & Clear day & \multirow{2}{*}{ B209 } & \multirow{2}{*}{ Off } & \multirow{2}{*}{ On } \\
\hline 7 & Cloudy day & & & \\
\hline
\end{tabular}
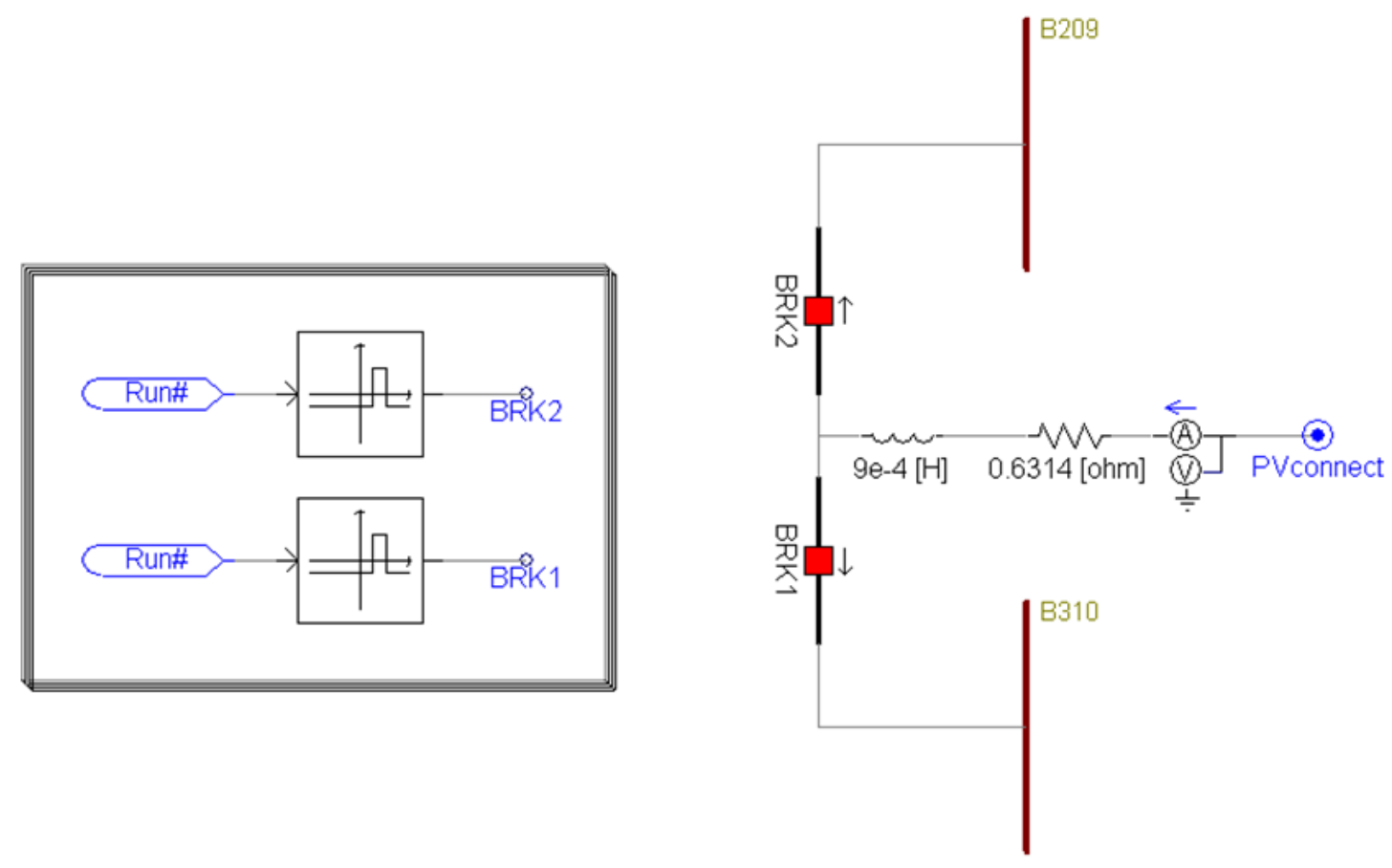

Figure 5-1: Schematic to allow the PV system to connect to different buses

In the interest of conserving time, this study only looks at a ten minute scenario that is further compressed into sixty seconds of simulation time. The feeder profile is a composite of eleven variable resistors located at various busses updated every second 
$(1 \mathrm{~Hz})$, representing ten second data resolution. In the span of ten minutes, the feeder load profile gradually increases from about 3.3MW to about 3.8MW as shown in Figure $5-2$.

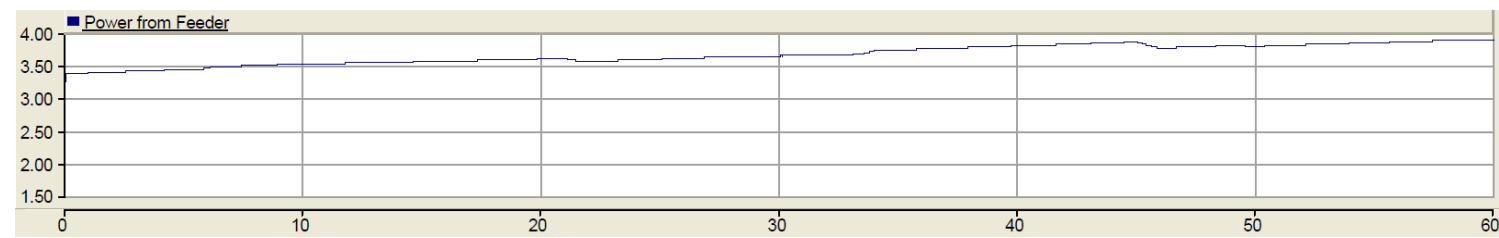

Figure 5-2: Feeder load profile with no PV system connected (run 1)

The solar profile was updated every tenth of a second (sampling rate of $10 \mathrm{~Hz}$ ), representing one second data resolution. The temperature was assumed to be constant for the entire ten minute period and was set to 25 degrees $C$. The PV system model was set up so that it would output about $2 \mathrm{MW}$ at $12.5 \mathrm{kV}$ when the radiation input is at $1000 \mathrm{~W} / \mathrm{m}^{2}$. To mimic a clear day, the radiation data increased from 985 to $990 \mathrm{~W} / \mathrm{m}^{2}$ in ten minutes. However, to simulate a partially cloudy day, the radiation data consisted of two dips as shown in Figure 5-3; the dips represent the panels being shaded twice in a ten minute span due to moving clouds. 


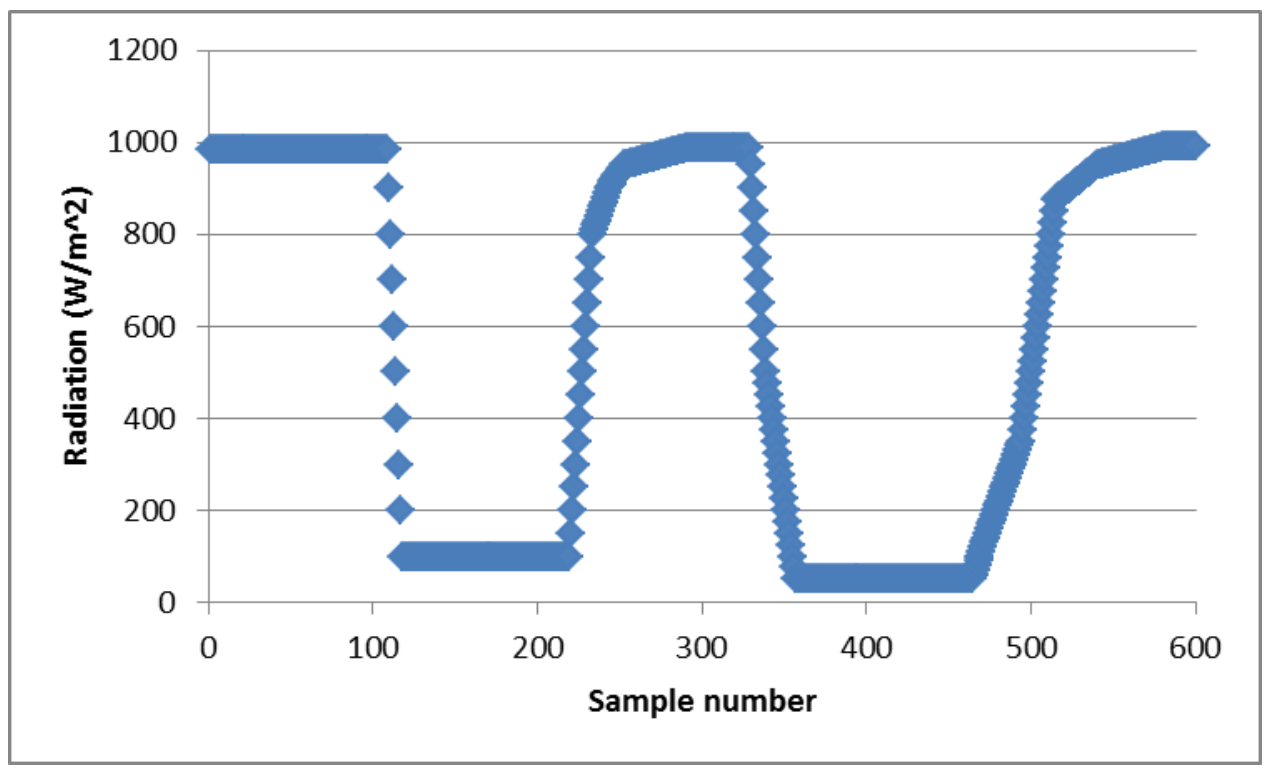

Figure 5-3: Solar profile representing a partially cloudy day

\subsection{Results}

Once the setup is complete, all seven runs can be simulated back to back automatically using the multiple run feature and the selected data will be recorded in output files. However, to better illustrate the results in this thesis, the multiple run was disabled and each run was individually simulated. The power supplied from the feeder, the power supplied by the PV system, and the voltage at all regulator busses for each of the seven runs are shown in Figure 5-4 through Figure 5-10. When the PV system is connected the bus voltages close to the interconnect are high initially until the regulators can fix it after the delay. This is neglected when recording the maximum voltage levels, as the recorders begin recording after an eight second delay thus allowing the regulators to change taps once if needed. 


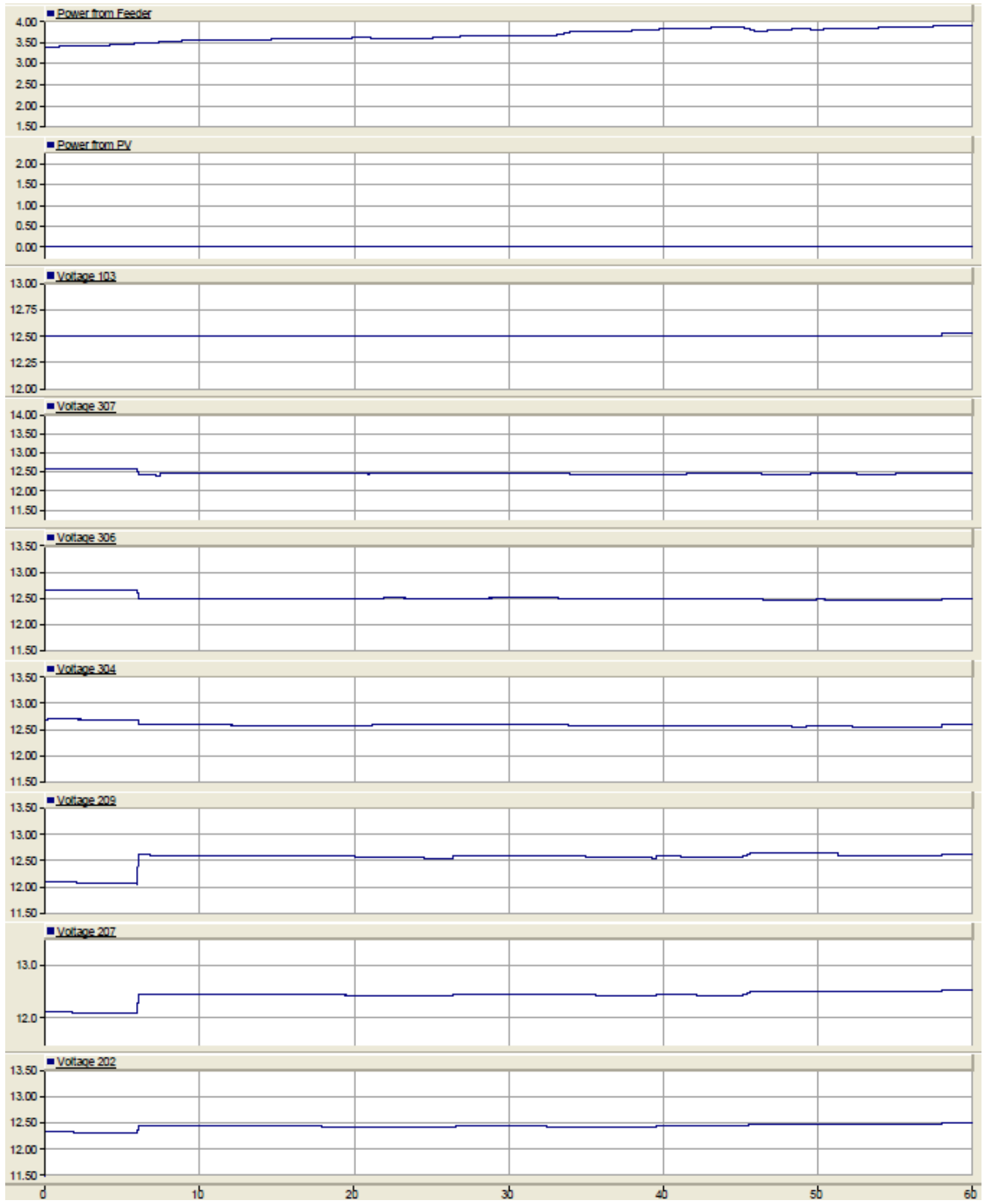

Figure 5-4: Power and voltage plots for run 1 


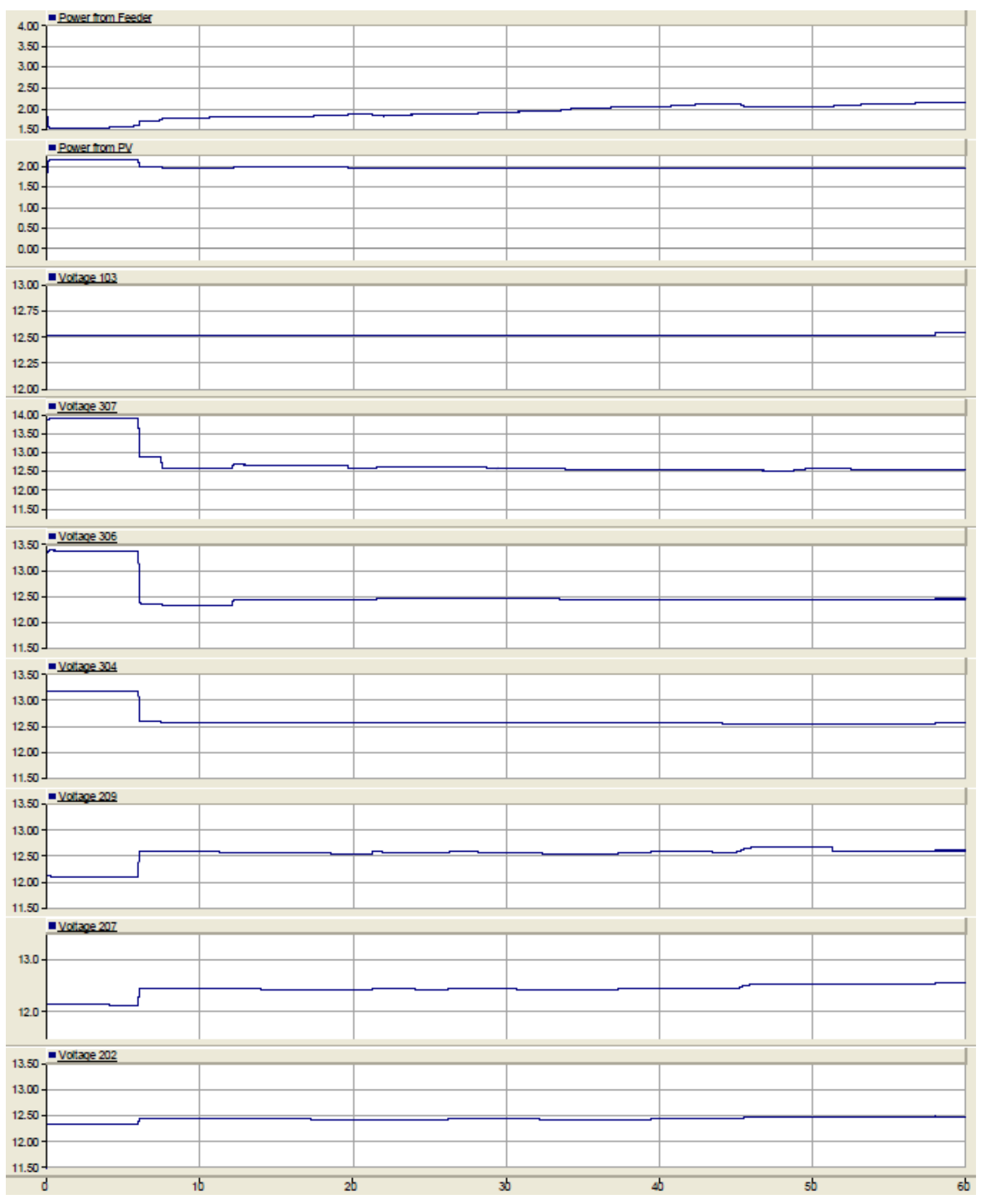

Figure 5-5: Power and voltage plots for run 2 


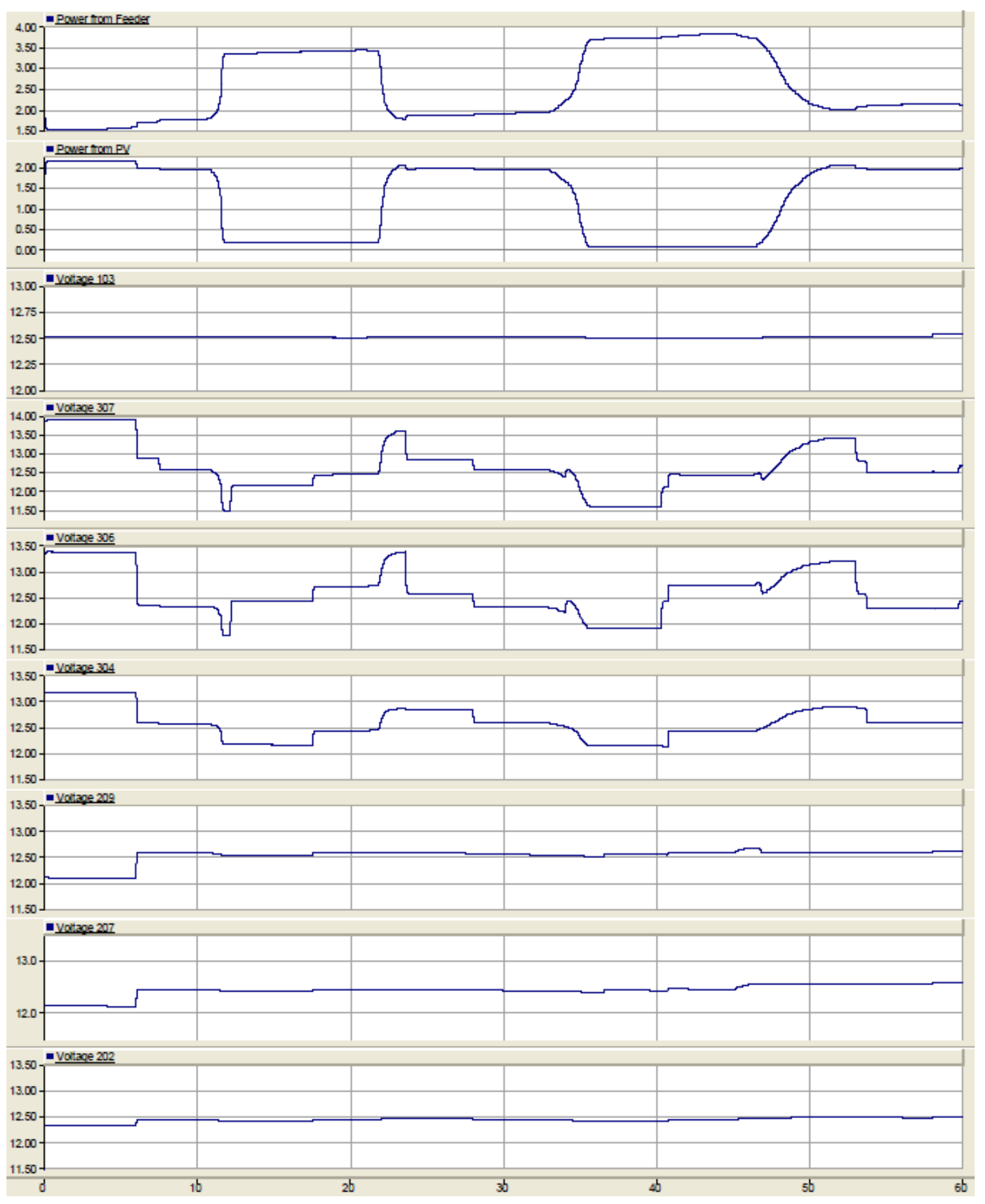

Figure 5-6: Power and voltage plots for run 3 


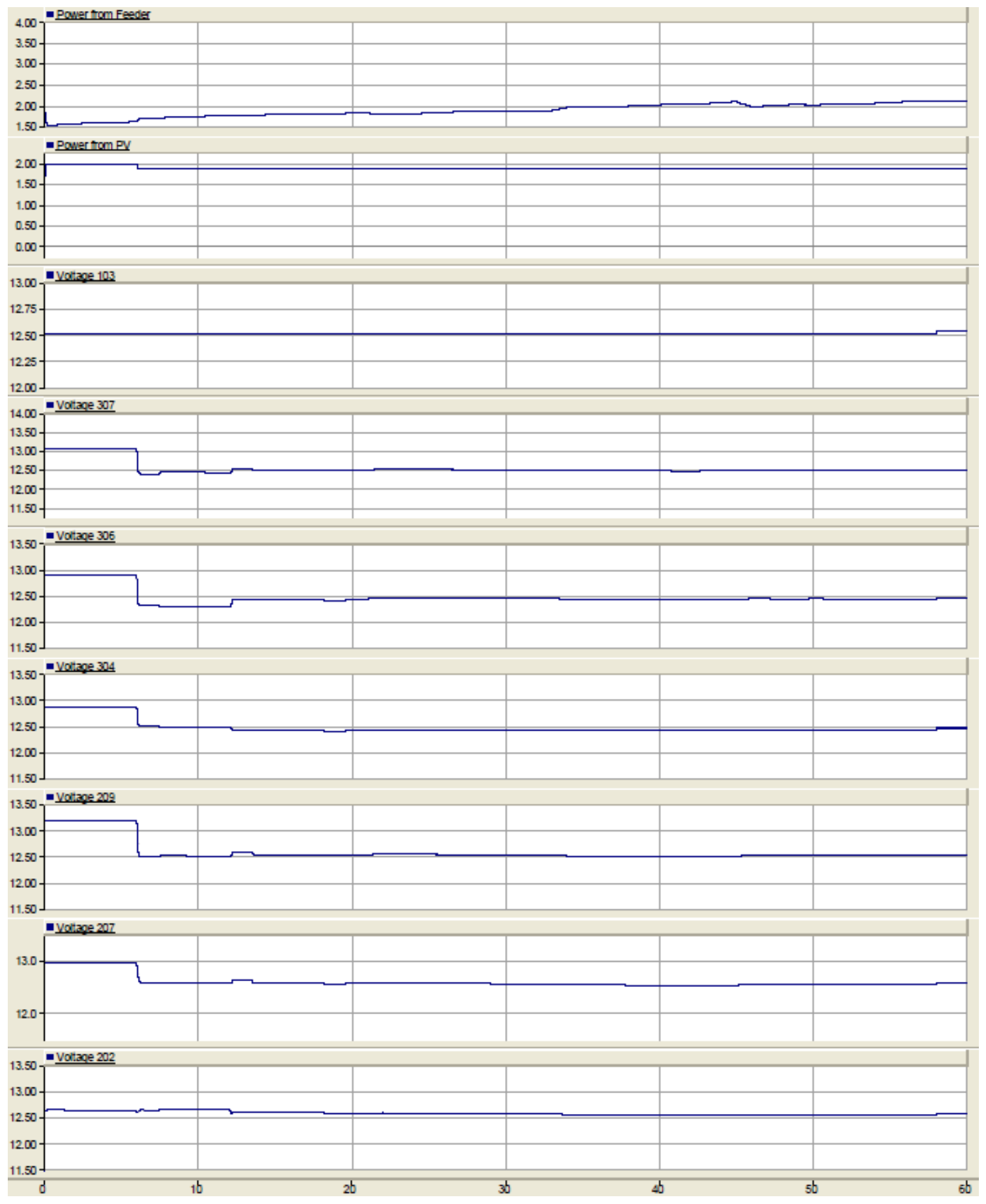

Figure 5-7: Power and voltage plots for run 4 


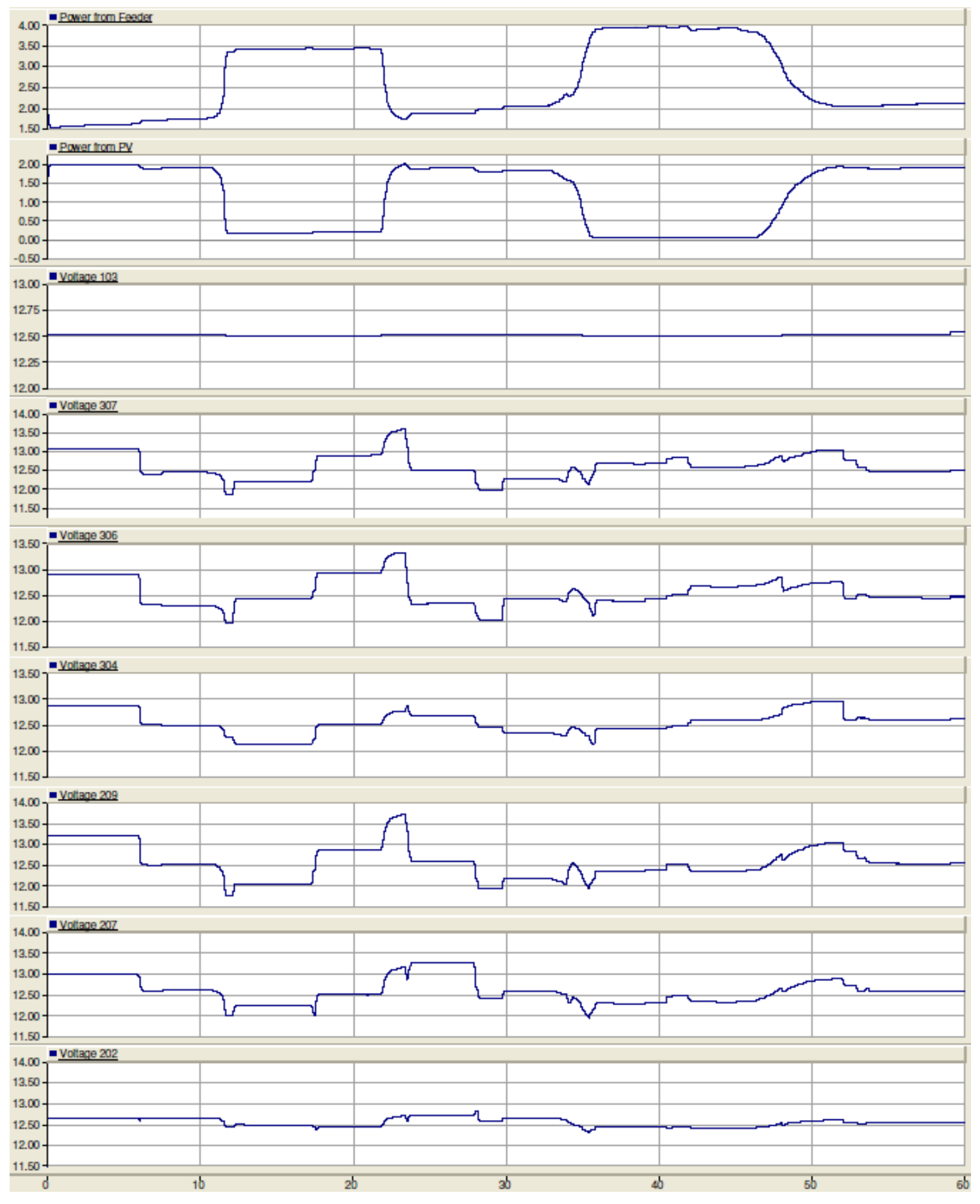

Figure 5-8: Power and voltage plots for run 5 


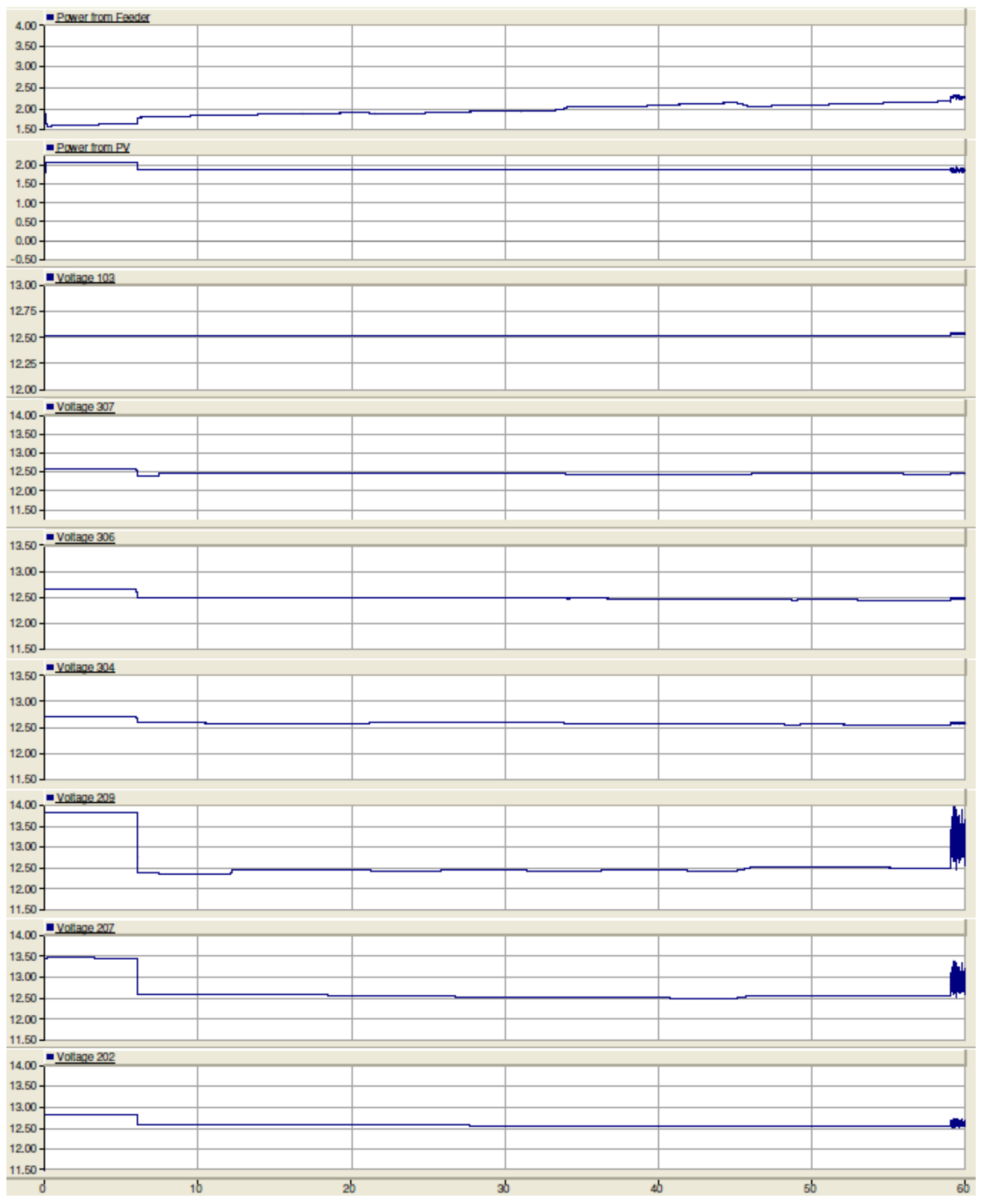

Figure 5-9: Power and voltage plots for run 6 


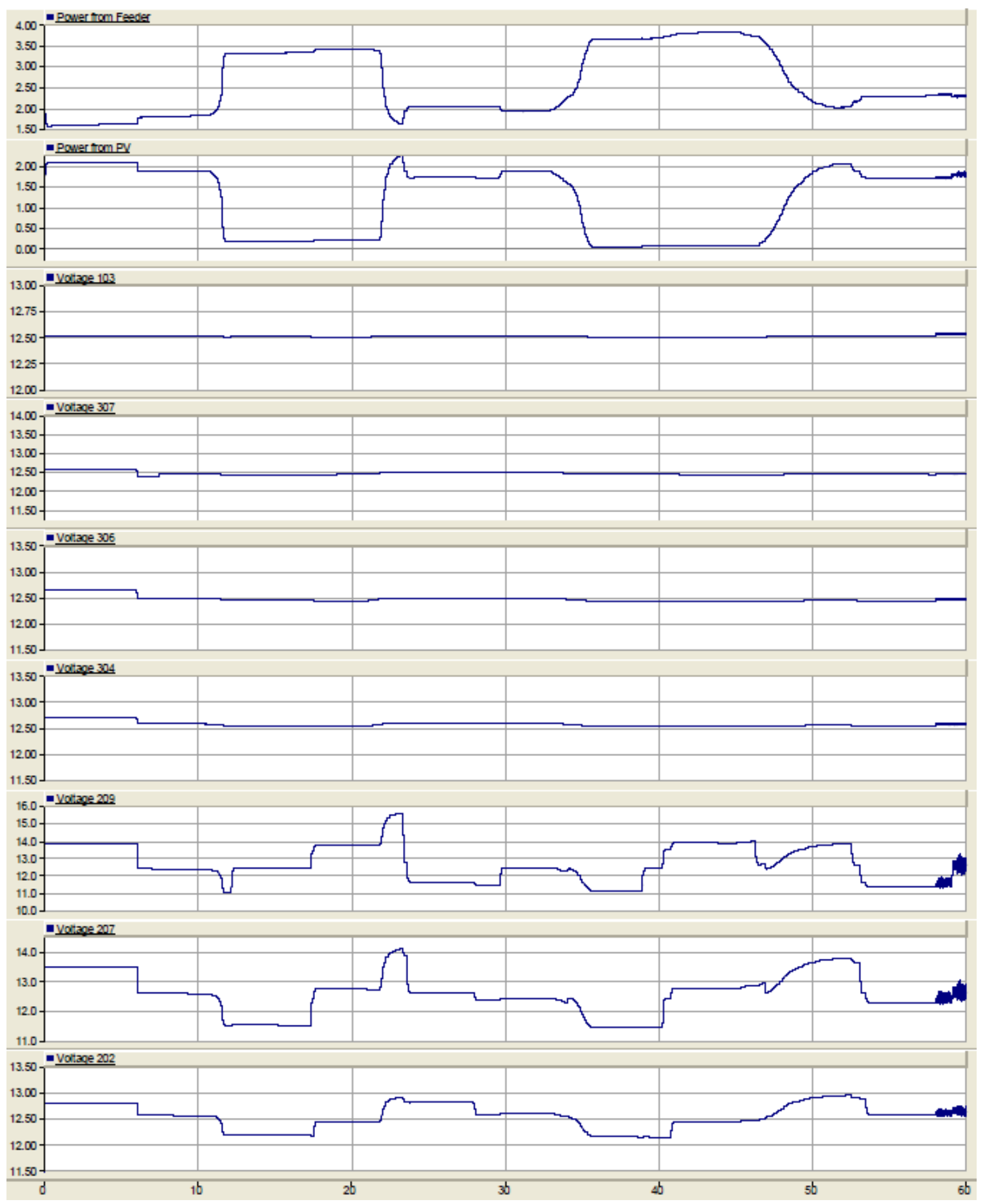

Figure 5-10: Power and voltage plots for run 7 
During runs 6 and 7 - when the PV system is connected to bus $209-\mathrm{a}$ disturbance appears on that lateral during last second or two of the simulation as seen in Figure 5-9 and Figure 5-10. This is due to a lack of reactive power support on that lateral. Adding a capacitor bank to that lateral and re-simulating resulted in eliminating the disturbance. However, in order to ensure that each option is being studied under similar condition, the decision was made to proceed without the capacitor bank. The only data that is significantly affected by the disturbance is the maximum voltage level for run 6. This was resolved by re-simulating run 6 for 59 seconds and using the resulting maximum voltage value.

The number of times a device changes taps for each run is presented in Table 5-2. Runs 3, 5, and 7 represent the partially cloudy days, and the effects of this are noticeable as the switching operations are higher for those runs. For runs 3 and 7 the PV system was connected to bus 310 and 209 respectively, and there is an increase in switching for the devices on the lateral that the PV system was connected, whereas the switching occurrence for the devices on the other lateral are similar to the clear day run. However, connecting the PV system to both busses for run 5 resulted in an increase in switching occurrence for all devices.

Table 5-2: Switching occurrences for each device

\begin{tabular}{|c|c|c|c|c|c|c|c|}
\hline Run \# & B103 & B307 & B306 & B304 & B209 & B207 & B202 \\
\hline 1 & 0 & 3 & 1 & 1 & 2 & 2 & 3 \\
\hline 2 & 0 & 3 & 3 & 1 & 2 & 3 & 3 \\
\hline 3 & 0 & 2 & 8 & 5 & 3 & 3 & 3 \\
\hline 4 & 0 & 1 & 3 & 3 & 1 & 2 & 3 \\
\hline 5 & 0 & 4 & 6 & 5 & 5 & 6 & 4 \\
\hline 6 & 0 & 2 & 1 & 1 & 4 & 1 & 1 \\
\hline 7 & 0 & 2 & 1 & 1 & 8 & 7 & 5 \\
\hline
\end{tabular}


Another important observation about Table 5-2 is that the substation LTC (103) does not switch for any run. This can be verified to be accurate by noting that the voltage at bus 103 is quite flat and stable no matter the condition of the rest of the circuit as shown in Figure 5-4 through Figure 5-10. This, however, is a sharp contrast in comparison to the results summarized in Table 3-2 from [24]. The reason for this inconsistency is the difference in control schemes. This thesis used the same voltage based controller for the substation LTC as was used on the other voltage regulators and is similar to the controller used in [24]; however, the controller in [24] is based on power i.e. the taps change bases on the amount of power flow through the LTC. This type of control scheme appears to be a poor choice given the fact that the PV system introduces significant power variations on a partially cloudy day resulting in excessive switching, especially when it seems that the bus voltage is quite flat. Unnecessary switching of the substation LTC could also cause additional switching by other devices thus further exacerbating the problem. In this study there is no switching activity by the LTC, so the data representing the LTC will be neglected.

\subsection{Analysis of Impact}

Once the simulation has run and the switching occurrence data are available, then the Circuit Impact Index can be calculated. The first step is to calculate the Device Cost Factor using equation (3-2). To be able to calculate the $D C F$, the annual average number of partially cloudy days is required. For the location of the proposed PV system, $D_{P C}=102$ days is assumed, therefore $D_{C l r}=365-102=263$ days. Using the first option (connecting the PV system to bus 310 ) as an example, the $D C F$ for the regulator at bus 307 is calculated as follows: 


$$
D C F_{307}=\frac{(3 * 263)+(2 * 102)}{3 * 365}=0.907
$$

Here we see that the $D C F$ is less than one, implying that the impact on the device will be reduced due the PV system being connected. It may be helpful to ensure that such a result is reasonable; in this situation the number of tap changes is the same without the PV system or with the PV system on a clear day, but the number reduces during a partially cloudy day. This may appear counterintuitive, but it should be noted that the delay for device 307 is longer than the other two devices ( $7.5 \mathrm{sec}$ compared to 6 sec) on the lateral, and also note that the number of switching for the other devices on the lateral does increase significantly on the partially cloudy day.

The next step is to calculate the Device's New Operating Cost. Before doing so the device $O C$ needs to be determined; recall the $O C$ is the estimated annual operational cost of the device before adding the PV system to the circuit. Since the substation LTC is neglected, it can be assumed that the rest of the voltage regulator devices are similar. However, just because the devices are similar, it does not imply that the $O C$ for each device will be the same. One needs to account for the fact that the further upstream a device is, the more customers will be impacted if the device fails - indicating greater loss of combined production time, possible damage to an increased number of sensitive equipment, etc. - therefore the utility may spend more so as to get the device in service faster. To account for this a simplifying assumption was made that the customers at each bus are equally important, and using device 209 as the base, each additional bus upstream adds 0.5 to the $O C$. For instance there are seven upstream busses from device 209 to device 202, and that adds an $O C$ of 3.5 to the base of 1 resulting in 4.5 for the $O C$ of device 202. Using this method the $O C$ for each of the devices are determined as shown in Table 5-3. 
Table 5-3: The OC determined for each device

\begin{tabular}{|c|c|c|c|c|c|c|}
\hline Device & 307 & 306 & 304 & 209 & 207 & 202 \\
\hline OC & 2 & 2.5 & 3.5 & 1 & 2 & 4.5 \\
\hline
\end{tabular}

Now that the $O C$ has been determined for each device, the Device's New Operating Cost can be calculated for the regulator at 307 as follows:

$$
N C_{307}=2 * 0.907=1.814
$$

An alternate way of understanding the significance of $N C$ is to consider it in monetary terms. Suppose device 307 has an annual cost of $\$ 2,000$ prior to the installation of the PV system; this includes the annual maintenance cost and a fraction of the replacement cost based on the expected life of the device. After the PV system is operational and connected to bus 301 , the annual cost of the device is expected to be $\$ 1,814$.

In a similar manner, the $D C F$ and $N C$ for the rest of the devices are calculated. The last step will be to calculate the Circuit Impact Index for that particular option. Recall the equation for the Circuit Impact Index is as follows:

$$
C I I=\frac{\sum N C \text { for all devices }}{\Sigma O C \text { for all devices }}
$$

The process would be repeated to calculate the $C I I$ for each option being studied. The results for this study can be found in Table 5-4. Notice how implementing any of the three options results of a $D C F$ for device 307 that is less than one, yet the $C I I$ for each option is greater than one indicating that the addition of the PV system to the circuit (without any additional mitigation) will result in an increased cost to the utility. 
Table 5-4: Summary of results for Cll study

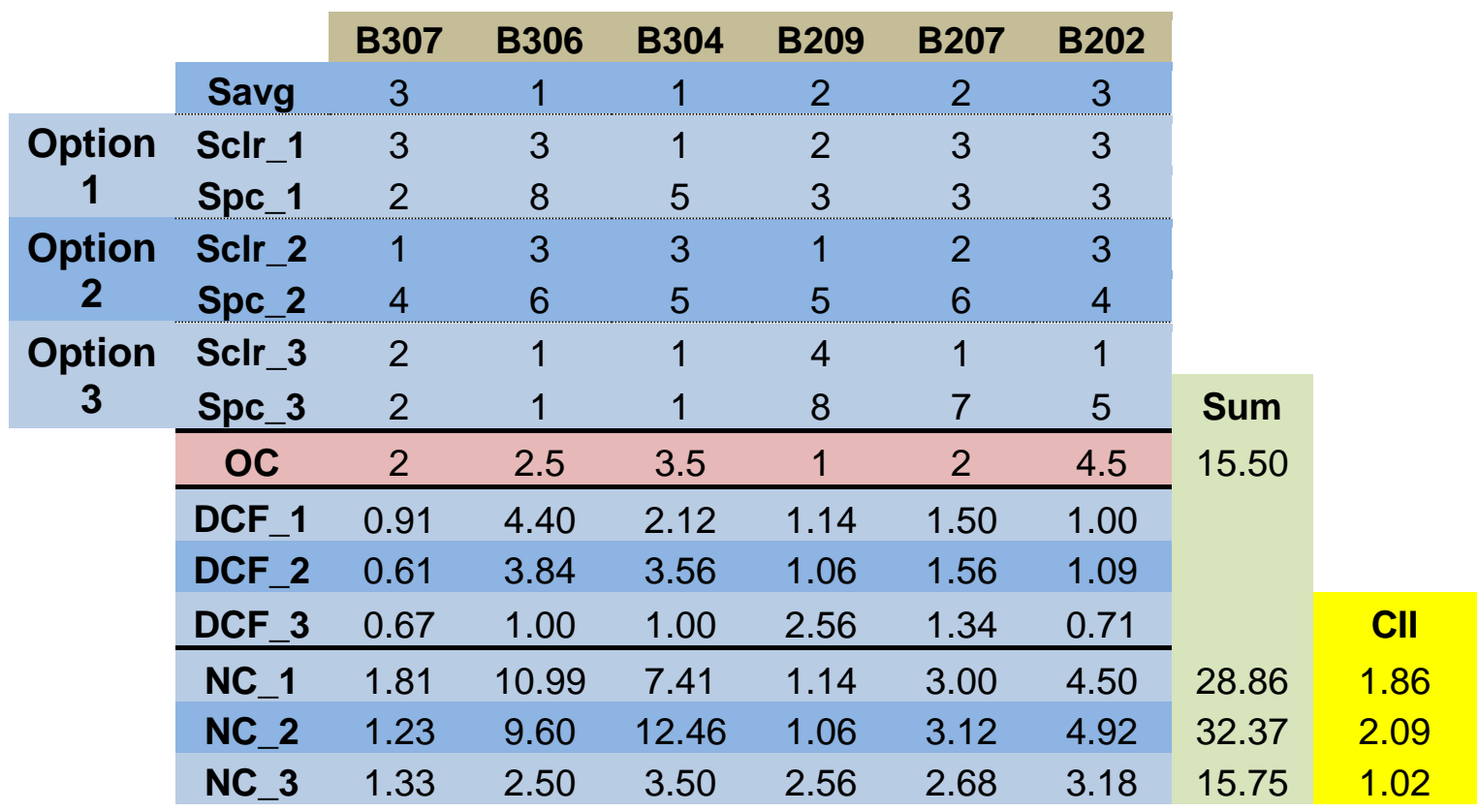

\subsection{Analysis of Voltage Quality}

Once again, the $C I I$ can be thought of in financial terms i.e. implementing option 1 with a $C I I=1.86$ will result in an almost doubling of the annual operating cost of the voltage regulating devices on the circuit. So one could claim that option 3 with the lowest CII would be the best option, but this is not yet known. The best option cannot be determined based solely on the value of $C I I$. To be able to make a well informed decision, the engineer performing the study needs to compare the lower CII to some metric for voltage quality. It is quite possible that a lower CII may come at a cost of a poorer voltage quality. Results of the two metrics must be compared side-by-side for reasonable tradeoffs before the engineer can make a good decision.

Selecting the so-called best option is a rather qualitative / subjective process that relies heavily on the engineer's experience and judgment. For a feeder supplying a residential load it might be satisfactory to select an option that sacrifices some voltage 
quality but has a significant lower CII. On the other hand, if the feeder is supplying a sensitive load such as a hospital or industrial area running precision equipment, then the best option would be one that has the highest voltage quality even if the equipment impact might be higher. The justification here is that trying to save operation cost could lead to much bigger negative impact to the utility if the low voltage quality causes loss of life, loss of production time, or damage to sensitive equipment.

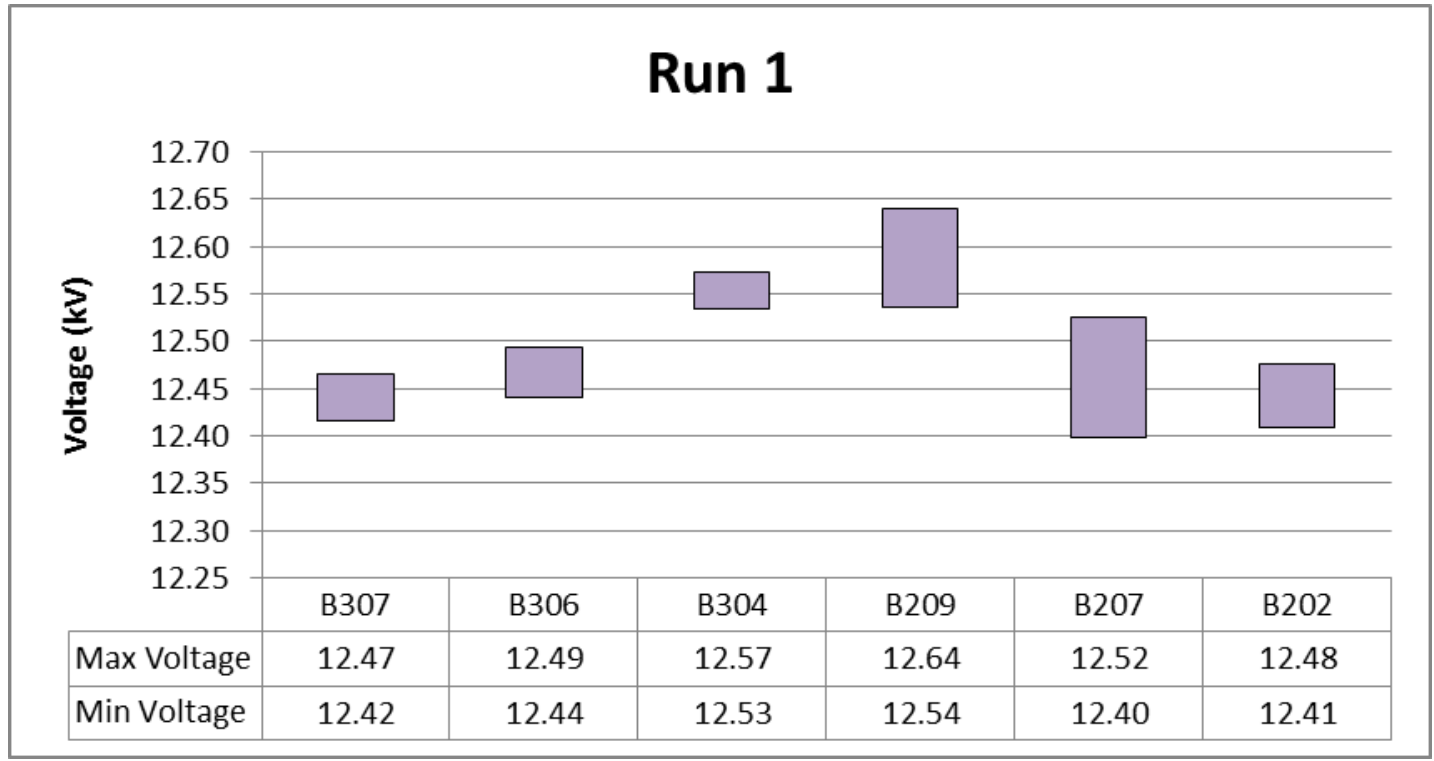

Figure 5-11: Voltage swing for no PV system connected

To determine the voltage quality, this study will observe the minimum and maximum voltage levels recorded for each run, and the amount of time the voltage was above or below certain user determined limits. Run 1 determines the characteristics of the distribution circuit prior to the addition of the PV system, and as shown in Figure 5-11 the voltage swings are fairly close to the nominal voltage of $12.5 \mathrm{kV}$. The largest deviation from nominal is less than 1.2\%. Similarly Figure 5-12, Figure 5-13, and Figure 5-14 show the voltage swings for runs 2,4 , and 6 respectively representing a clear day scenario for each option. With the PV system connected to the distribution circuit, notice the voltage spread is slightly greater, yet within acceptable limits as the deviations do not 
exceed $2 \%$. Based on the observations thus far, all options seem viable, with option 3 being the best choice because it has the lowest $C I I$.

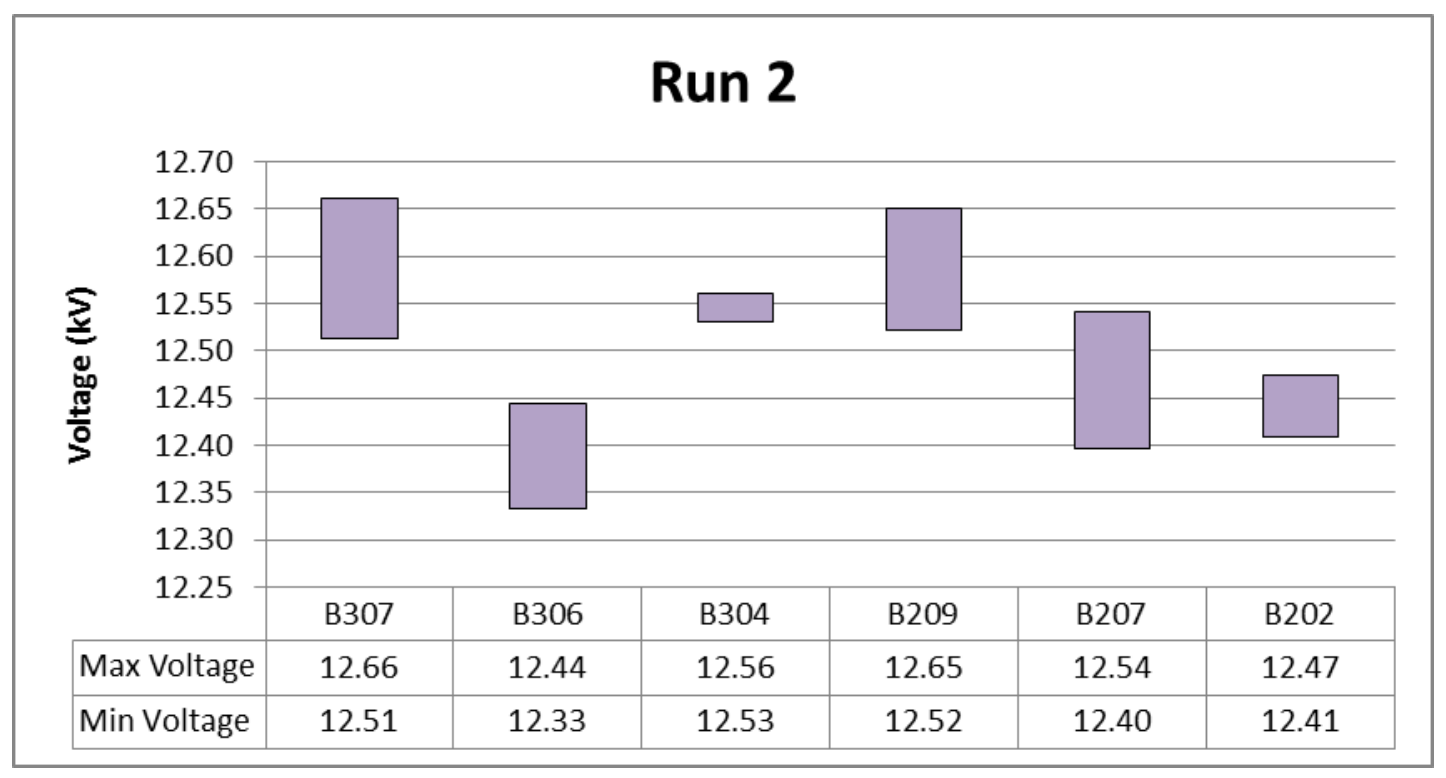

Figure 5-12: Voltage swing for option 1 clear day

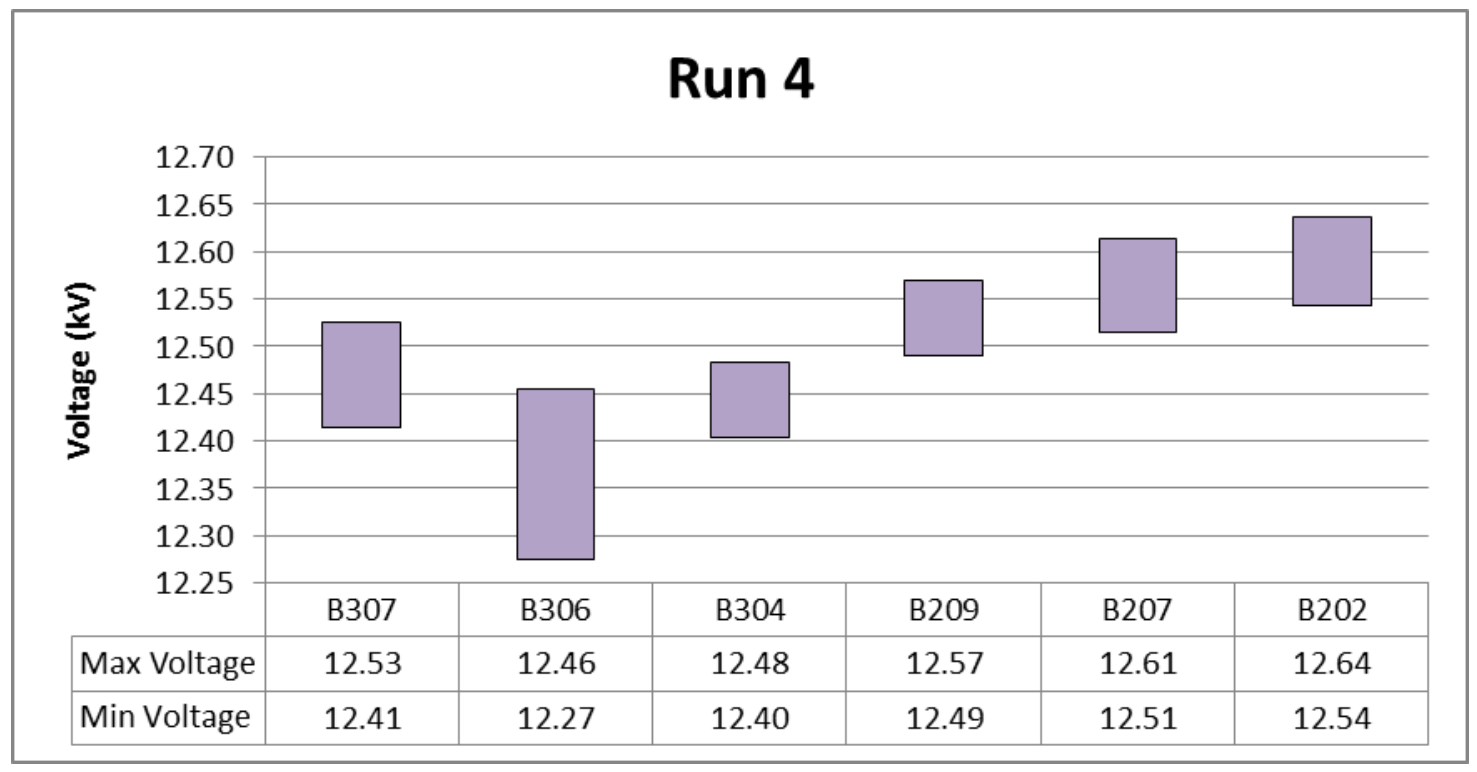

Figure 5-13: Voltage swing for option 2 clear day 


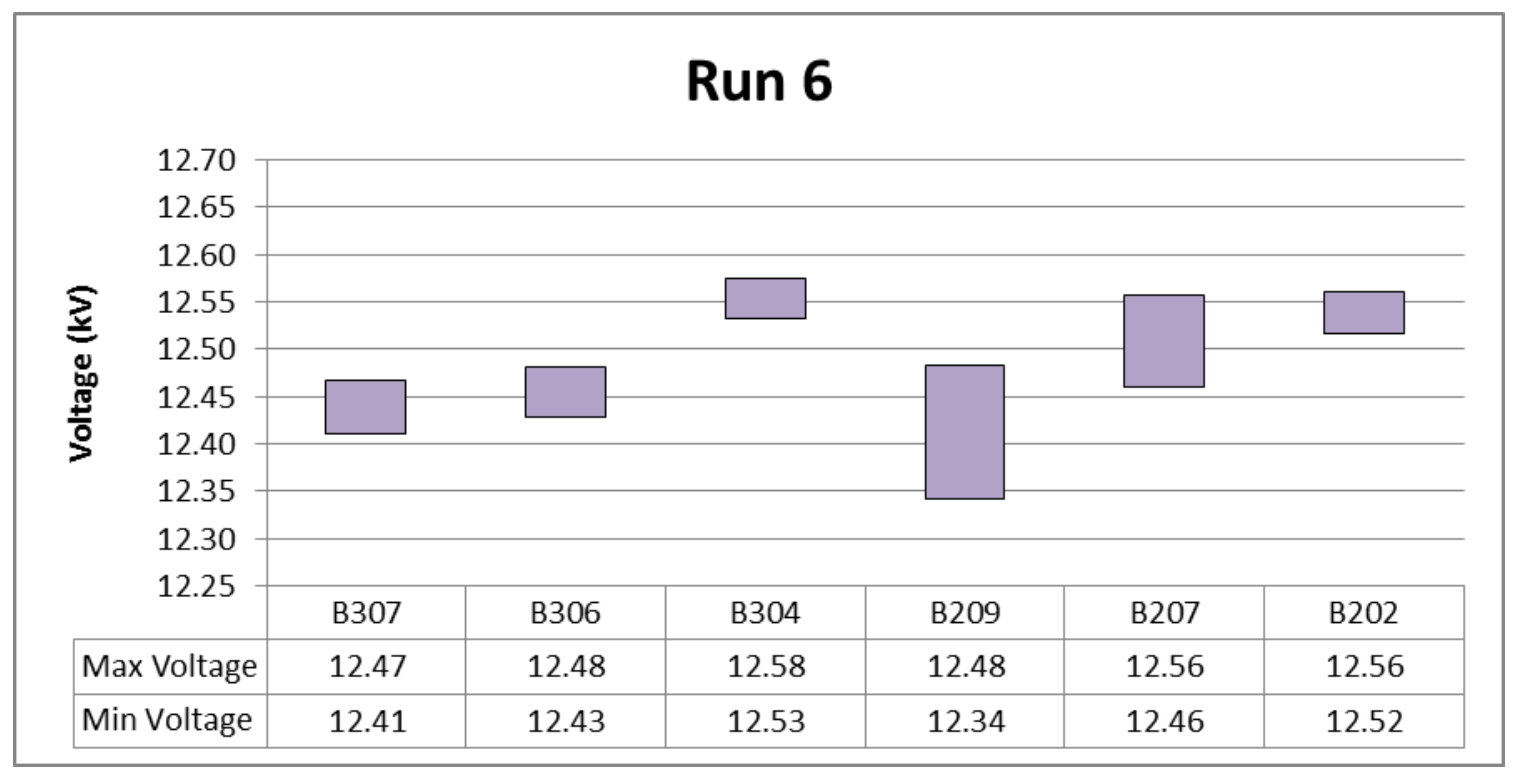

Figure 5-14: Voltage swing for option 3 clear day

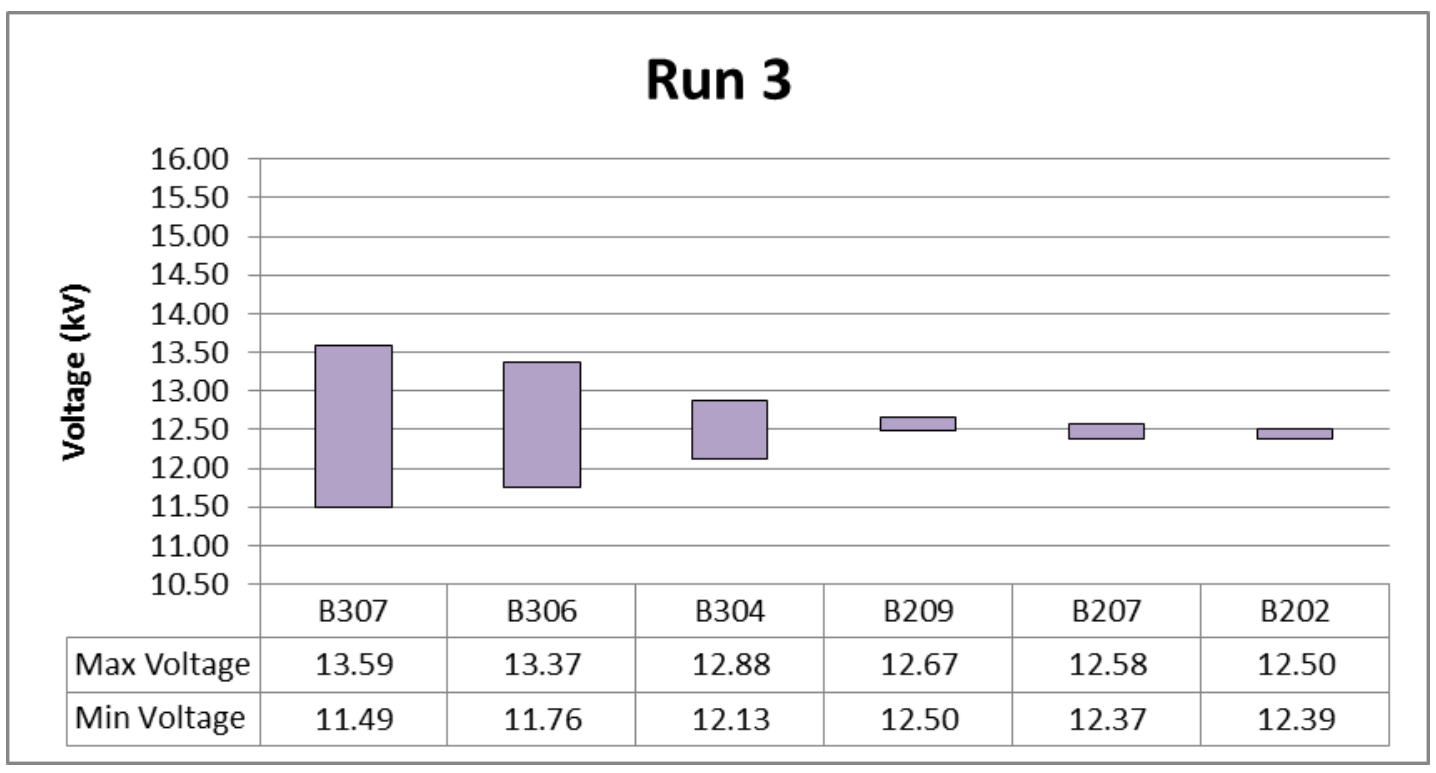

Figure 5-15: Voltage swing for option 1 partially cloudy day

The next task is to observe the effect of partially cloudy days on the voltage swings. For run 3 the PV system is connected to bus 310 and Figure 5-15 clearly shows the variability introduced by the PV system, as the bus closest to the interconnect (307) has the largest swings that decrease as it gets closer to the substation, whereas the 
other lateral has a fairly stable voltage. The PV system is connected to both bus 310 and 209 for run 5, and the behavior is predictable as observed in Figure 5-16. The voltage swings appear on both laterals, and the deviation decreases in the upstream direction. Run 7 has similar results as run 3, however, since the PV system is connected to the other (upper) lateral, the fluctuation are also present on the other lateral as seen in Figure 5-17. The key difference for run 7 is that the magnitudes of deviations are much large. One possible explanation for the increase in deviation magnitude could be because of the lack of reactive power (VAR) support on the upper lateral. After observing the voltage swing characteristics, based on the chosen model of the circuit, it seem that option 3 may not be a good option unless some mitigation is implemented. Between options 1 and 2, option 1 seems to be the best choice because the fluctuations only appear on one lateral and thus might be easier to mitigate. Although it is possible that due to the loop created by option 2 , the same mitigation could return better results as compared to option 1 ; this would require further study of the mitigation options.

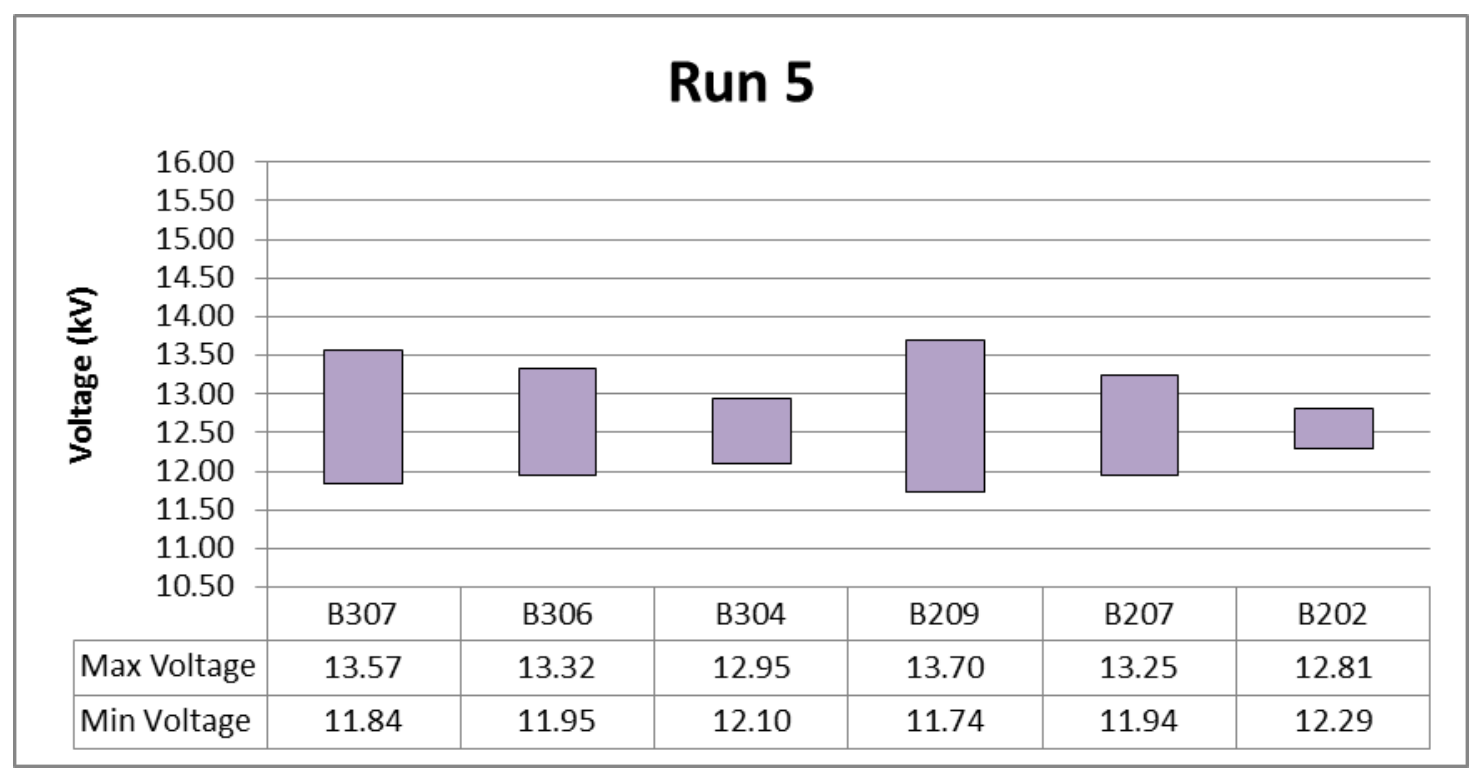

Figure 5-16: Voltage swing for option 2 partially cloudy day 


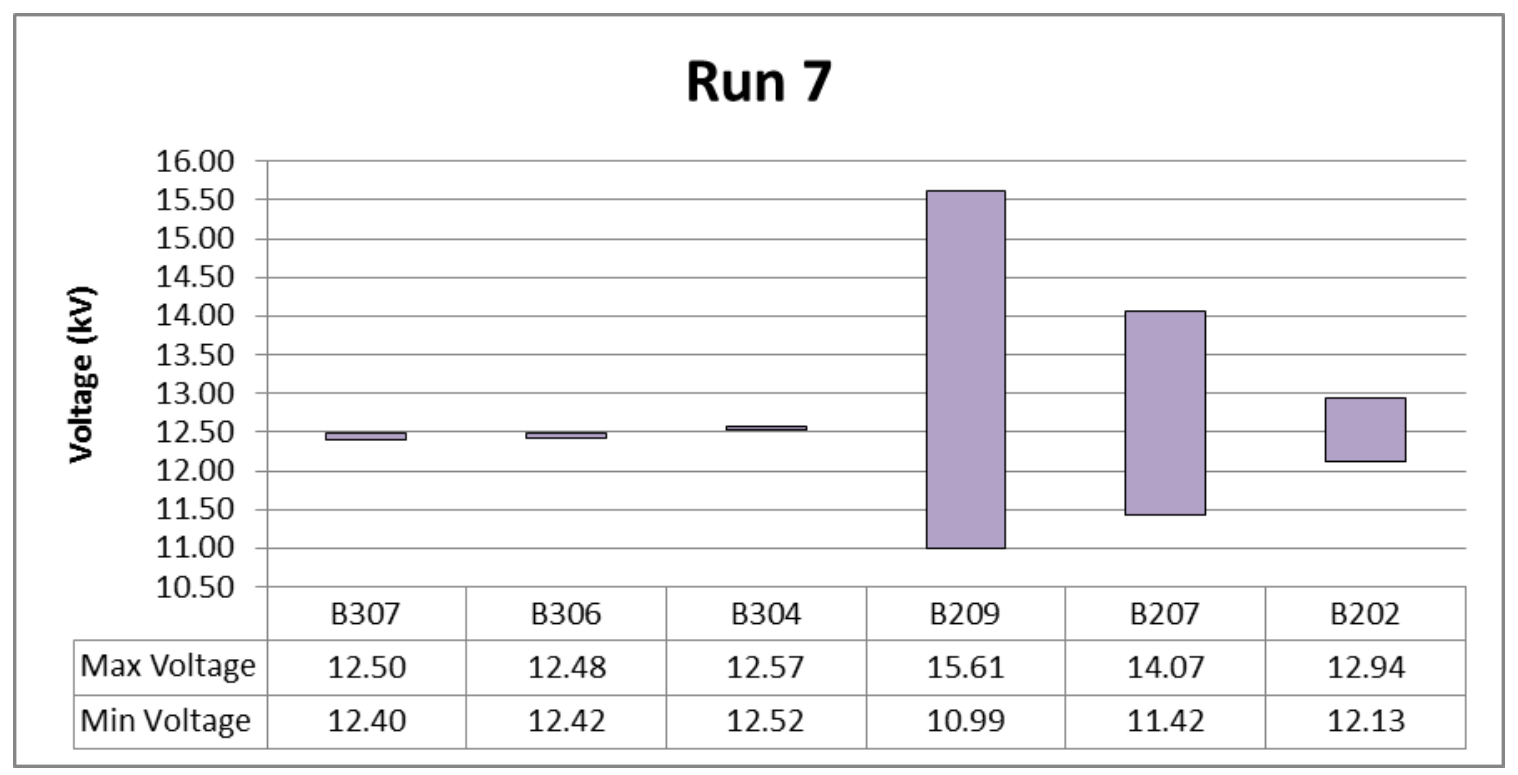

Figure 5-17: Voltage swing for option 3 partially cloudy day

Lastly let's consider the time periods that the voltage is outside tolerance. For this study, the voltage limits were set to $+/-5 \%$, and Table $5-5$ represents the periods that the voltage was over (OV) or under (UV) the specified limits. The voltage is within limits for most of the runs, and other than the short duration of over voltage during run 6 (possibly due to lack of VAR support), the voltage limits are violated during runs simulating partially cloudy days Figure 5-18, Figure 5-19, and Figure 5-20 present a graphical view of the total time duration for the over voltage and under voltage conditions that occurred for the partially cloudy days of the three options. Run 5 has the lowest cumulative time, but it affects more of the circuit than either run 3 or 7 . Run 3 has a lower cumulative time compared to run 7 . Once more, option 1 or option 2 might be a good option. 
Table 5-5: Time period for over / under voltage condition

\begin{tabular}{|ccccccc|}
\hline Run & B307 & B306 & B304 & B209 & B207 & B202 \\
\hline 1 OV & 0 & 0 & 0 & 0 & 0 & 0 \\
\hline 1 UV & 0 & 0 & 0 & 0 & 0 & 0 \\
\hline 2 OV & 0 & 0 & 0 & 0 & 0 & 0 \\
\hline 2 UV & 0 & 0 & 0 & 0 & 0 & 0 \\
\hline 3 OV & 5.44 & 4.56 & 0 & 0 & 0 & 0 \\
\hline 3 UV & 5.77 & 0.52 & 0 & 0 & 0 & 0 \\
\hline 4 OV & 0 & 0 & 0 & 0 & 0 & 0 \\
\hline 4 UV & 0 & 0 & 0 & 0 & 0 & 0 \\
\hline 5 OV & 1.55 & 1.40 & 0 & 1.59 & 4.59 & 0 \\
\hline 5 UV & 0.46 & 0 & 0 & 0.53 & 0 & 0 \\
\hline 6 OV & 0 & 0 & 0 & 0.48 & 0.06 & 0 \\
\hline 6 UV & 0 & 0 & 0 & 0 & 0 & 0 \\
\hline 7 OV & 0 & 0 & 0 & 16.31 & 6.53 & 0 \\
\hline 7 UV & 0 & 0 & 0 & 16.73 & 11.03 & 0 \\
\hline
\end{tabular}

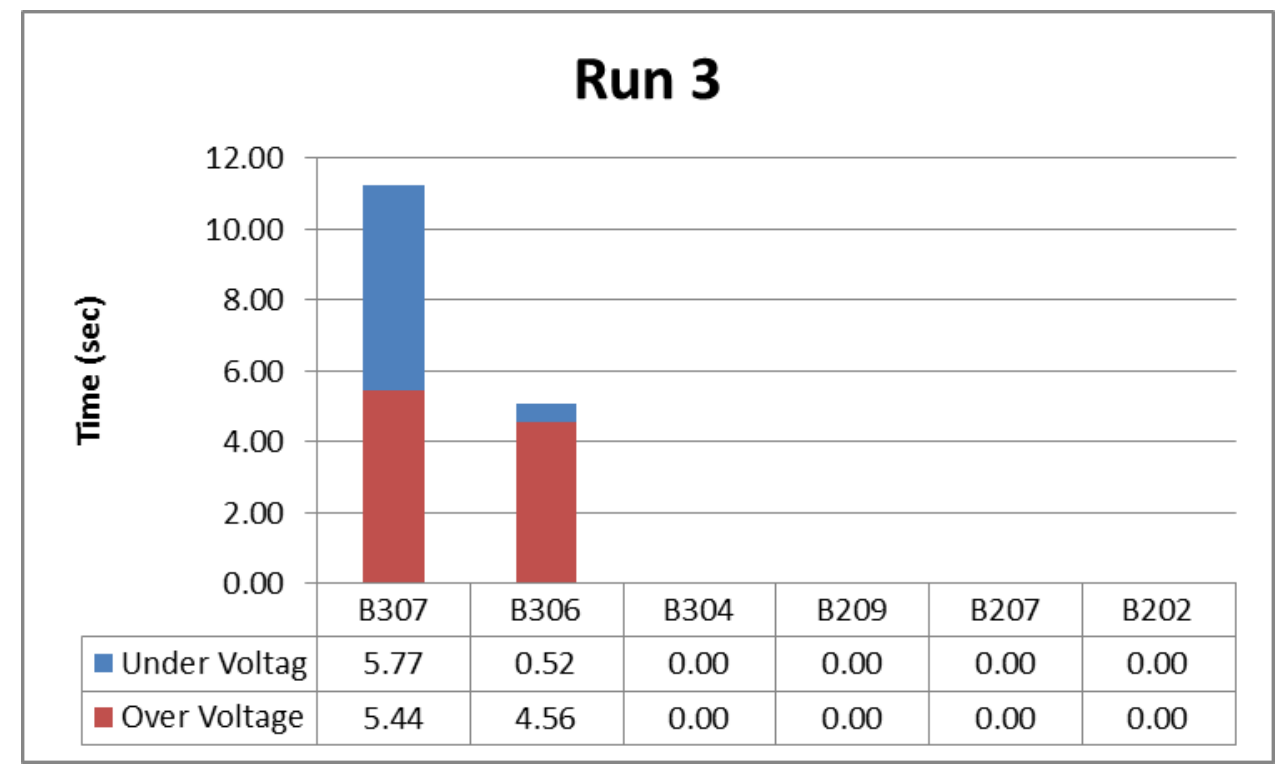

Figure 5-18: Duration for out of limit voltage for run 3 


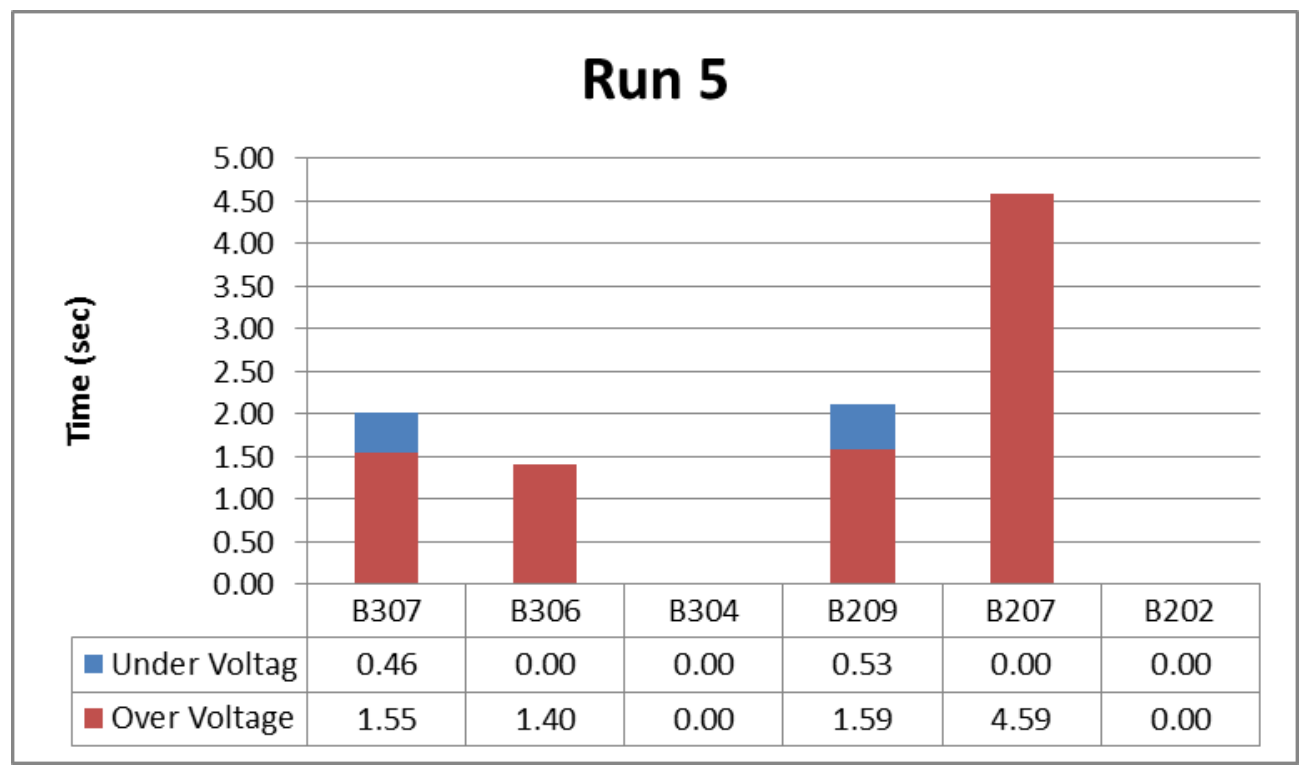

Figure 5-19: Duration for out of limit voltage for run 5

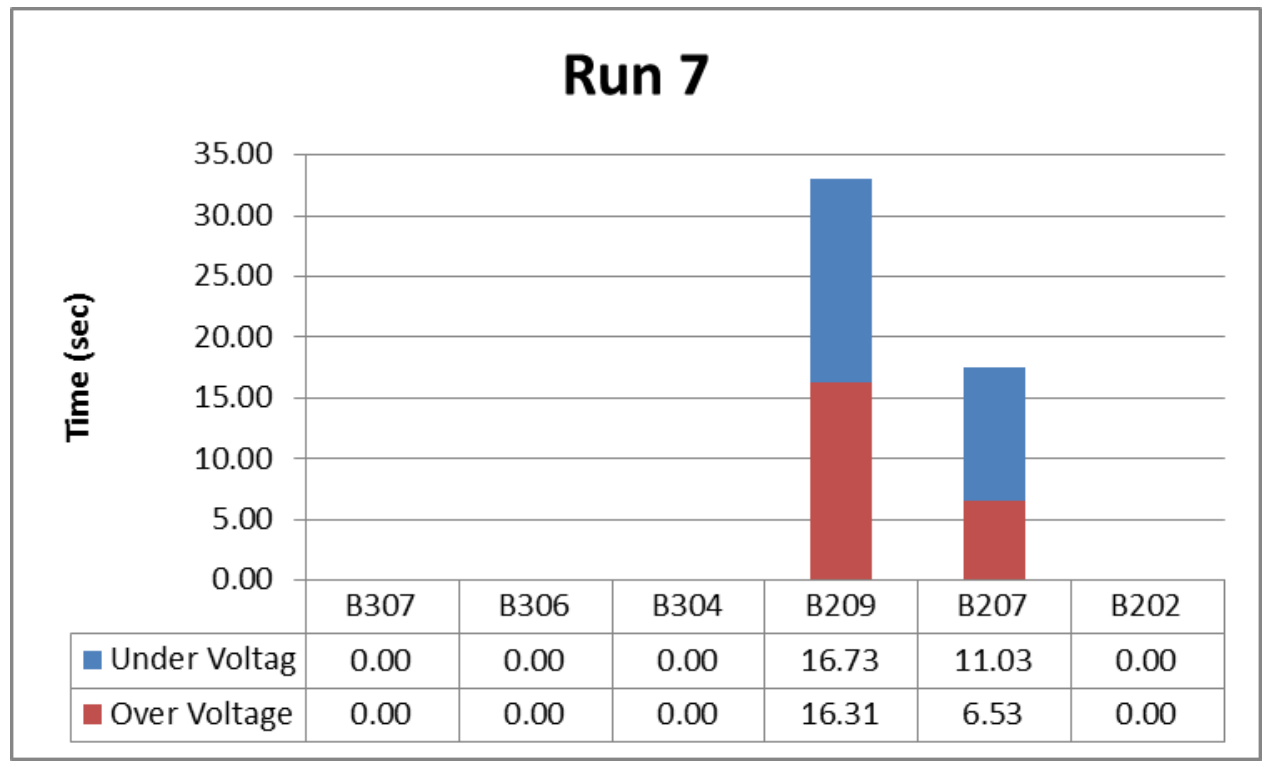

Figure 5-20: Duration for out of limit voltage for run 7 


\subsection{Summary}

Based on the discussion in this chapter, there are several factors that must be considered to determine the optimum interconnection connection for the PV system. The CII is an important metric in the decision making process, but it is not the only metric to base a decision on. The engineer needs to consider the voltage quality of the power being supplied to the customer, and realize that there exist a tradeoff between the voltage quality and the impact to the service life of the voltage regulation equipment. Once the $C I I$ and voltage quality for different options are evaluated, only then can the engineer make an informed decision regarding the option to implements; here too, the decision is unique to the particular circuit and the customers being served by it. For the three options discussed in this chapter, and assuming a mostly residential load on the circuit, connecting the PV system to bus 310 (option 1) is the best choice given that it has a better voltage quality than option 3 and a lower CII than option 2 . 


\section{Conclusion and Future Work}

The number and size of grid connected PV systems is expected to increase for at least the foreseeable future. As efficiency and service life of PV systems continue to improve, the environmental benefits are increased and the energy break-even point is reduced. However, much of the existing infrastructure was not designed to be able to accept distributed generation, especially one with as much intermittency as PV, yet government policies incentivize PV systems while making the utilities accept more interconnects than what may be ideal for a particular circuit. To allow the utility to determine the impact that such interconnects can have on voltage regulating devices, a metric is proposed and explored in this thesis.

The metric proposed not only provides insight into the impact on voltage regulation equipment thus allowing for optimal level of $\mathrm{PV}$ penetration on a given circuit, but it also allows the engineer(s) to weigh various mitigation options and locations to determine the mitigation technology and placement that provides the best return on investment. The example study in this thesis explored the ability of using the proposed metric to determine the optimum location for interconnecting a proposed PV system to the distribution circuit. The same methods can also be used to examine various mitigation options.

The index proposed in this thesis does not integrate the voltage quality into it, but rather compares it side-by-side. Future work should be done to determine a way to incorporate the voltage quality information into the impact index, while ensuring that the index still maintains an intuitive feel. Since determining acceptable voltage quality is rather subjective, the acceptable voltage quality and tradeoffs would need to be determined for the particular circuit being studied, and that can then be factored into the 
index calculation. The end result should be a single value for each option that provides insight into the impact, voltage quality, and the tradeoffs between the two.

The voltage regulating device used in this thesis is a standard transformer type line voltage regulator, yet there are many power electronics based FACTS type devices that can be used to regulate the voltage and mitigate fluctuations. Unfortunately many of the models for such devices are quite complex and require considerable computing power for simulations lasting longer than a few tens of cycles, which makes it extremely difficult to study mitigation options based on the use of such devices. This problem can be solved by creating models that accurately mimic the behavior and characters of such devices, but without using complex simulation components such as power electronics.

The accuracy of the study is highly dependent on the accuracy of the data used for the solar and feeder load profiles. Consequently, it would be helpful to collect and archive such data, as this would allow an engineer to create and study various scenarios as the need arises. Similarly, it would be beneficial to collect some "before and after" data for a particular PV system installation, and compare it to the predictions of the study to validate the models being used and "calibrate" the method if necessary.

Lastly, research needs to be done to make the method and index more robust for interconnecting additional PV systems to circuits that already have modest PV penetration. This would require creating statistical models that could predict the correlation of cloud shading for PV systems at different physical locations based on local weather information such as typical wind speed, cloud size etc. and the distance between the two PV system. 


\section{Bibliography}

[1] U.S. Energy Information Administration, "Annual Photovoltaic Cell/Module Shipments Report," U.S. Energy Information Administration, Washington DC, 2012.

[2] M. Olken, "The Sun Keeps Shining - updates on large-scale solar energy," IEEE power and energy magazine, vol. 11, no. 2, pp. 18-21, March/April 2013.

[3] B. Kroposki and K. Lynn, "Still Shining - solar energy is becoming mainstream," IEEE power and energy magazine, vol. 11, no. 2, pp. 18-21, March/April 2013.

[4] U.S. Energy Information Administration, "Electric Power Annual 2011," U.S. Department of Energy, Washington DC, 2013.

[5] Public Utilities Commission, "RPS Program Overview," CA.gov, 1 Feb 2012. [Online]. Available: http://www.cpuc.ca.gov/PUC/energy/Renewables/overview. [Accessed 11 Feb 2013].

[6] U.S. Energy Information Administration, "How much of our electricity is generated from renewable energy?," 27 June 2012. [Online]. Available:

http://www.eia.gov/energy_in_brief/article/renewable_electricity.cfm\#ftnote. [Accessed 22 March 2013].

[7] G. Pyo, H. W. Kang and S. Moon, "A new operation method for grid-connected PV system considering voltage regulation in distribution system," in Power and Energy Society General Meeting - Conversion and Delivery of Electrical Energy in the 21st Century, 2008.

[8] J. C. Rudolf, "The Parking Lot as 'Solar Grove'," The New York Times, 6 July 2010. [Online]. Available: http://green.blogs.nytimes.com/2010/07/06/the-parkinglot-as-solar-grove/. [Accessed 5 April 2013].

[9] R. Belur, "Microinverter Advantages," [Online]. Available: http://www.altenergymag.com/emagazine.php?art_id=1610. [Accessed 5 Apirl 2013].

[10] IEEE Std 1547-2003, IEEE Standard for Interconnecting Distributed Resources With Electric Power Systems, IEEE, 2003.

[11] R. A. Messenger and J. Ventre, Photovoltaic Systems Engineering, 3rd ed., Boca Raton, Florida: CRC Press, 2010. 
[12] IEEE Std 1547.2-2008, IEEE Application Guide for IEEE Std 1547, IEEE Standard for Interconnecting Distributed Resources with Electric Power Systems, IEEE, April 152009.

[13] NEMA Communications Department, "NEMA News," National Electrical Manufacturers Association, 1 January 2012. [Online]. Available:

http://www.nema.org/News/Pages/NEMA-Publishes-ANSI-C84-1-Electric-PowerSystems-and-Equipment-Voltage-Ratings.aspx. [Accessed 5 April 2013].

[14] T. A. Short, Electric Power Distribution Equipment and Systems, Boca Raton: CRC Press, 2006.

[15] S. Steffel and A. Dinkel, "Absorbing the Rays," IEEE power and energy magazine, vol. 11, no. 2, pp. 45-54, March/April 2013.

[16] N. W. Miller, R. A. Walling and A. S. Achilles, "Impact of distributed resources on system dynamic performance," in ransmission and Distribution Conference and Exposition, 2001.

[17] C. Trueblood, S. Coley, T. Key, L. Rogers, A. Ellis, C. Hansen and E. Philpot, "PV Measures Up for Fleet Duty," IEEE power and energy magazine, vol. 11, no. 2, pp. 33-44, March/April 2013.

[18] K. Turitsyn, P. Šulc, S. Backhaus and M. Chertkov, "Distributed control of reactive power flow in a radial distribution circuit with high photovoltaic penetration," in Power and Energy Society General Meeting, July 2010.

[19] A. S. Pabla, Electric Power Distribution, New York: McGraw-Hill, 2005.

[20] Cooper Power Systems, "Voltage Regulators, Intelligent Solutions. Endless Possibilities," [Online]. Available:

http://www.cooperindustries.com/content/dam/public/powersystems/resources/libr ary/225_VoltageRegulators/B22597020.pdf. [Accessed 8 April 2013].

[21] IEEE, "IEEE Guide for Identifying and Improving Voltage Quality in Power Systems," IEEE, March 312011.

[22] Cooper Power Systems, "CL-6 Series Control Installation, Operation, and Maintenance Instructions," October 2012. [Online]. Available:

http://www.cooperindustries.com/content/dam/public/powersystems/resources/libr ary/225_VoltageRegulators/S225111.pdf. [Accessed 174 2013].

[23] Cooper Power Systems, "QD3 Quik-Drive Voltage Regulator Tap Changer Manual," August 2012. [Online]. Available:

http://www.cooperindustries.com/content/dam/public/powersystems/resources/libr 
ary/225_VoltageRegulators/S225121.pdf. [Accessed 8 April 2013].

[24] M. Shao and J. Bebic, "San Diego Gas and Electric, 2 MW Photovoltaic Mitigation Project," GE Energy, New York, 2012. 


\section{Appendix A: Solar Profile Data}

The following is the user defined solar profile data that was fed into the PV system model via the Input.txt file:

Note: The page has been split into three columns to better accommodate the data.

\begin{tabular}{|c|c|c|c|c|c|c|c|c|c|c|c|}
\hline ! $\mathrm{Cl}$ & $\mathrm{Cl}$ & & & 985 & 25 & 985 & 25 & 986 & 25 & 100 & 25 \\
\hline & $\mathrm{Pa}$ & dyRa & & 985 & 25 & 985 & 25 & 986 & 25 & 100 & 25 \\
\hline & $\mathrm{Pa}$ & dyTe & & 985 & 25 & 985 & 25 & 986 & 25 & 100 & 25 \\
\hline 985 & 25 & 985 & 25 & 985 & 25 & 985 & 25 & 986 & 25 & 100 & 25 \\
\hline 985 & 25 & 985 & 25 & 985 & 25 & 985 & 25 & 986 & 25 & 100 & 25 \\
\hline 985 & 25 & 985 & 25 & 985 & 25 & 985 & 25 & 986 & 25 & 100 & 25 \\
\hline 985 & 25 & 985 & 25 & 985 & 25 & 985 & 25 & 986 & 25 & 100 & 25 \\
\hline 985 & 25 & 985 & 25 & 985 & 25 & 985 & 25 & 986 & 25 & 100 & 25 \\
\hline 985 & 25 & 985 & 25 & 985 & 25 & 985 & 25 & 986 & 25 & 100 & 25 \\
\hline 985 & 25 & 985 & 25 & 985 & 25 & 985 & 25 & 986 & 25 & 100 & 25 \\
\hline 985 & 25 & 985 & 25 & 985 & 25 & 985 & 25 & 986 & 25 & 100 & 25 \\
\hline 985 & 25 & 985 & 25 & 985 & 25 & 985 & 25 & 986 & 25 & 100 & 25 \\
\hline 985 & 25 & 985 & 25 & 985 & 25 & 985 & 25 & 986 & 25 & 100 & 25 \\
\hline 985 & 25 & 985 & 25 & 985 & 25 & 985 & 25 & 986 & 25 & 100 & 25 \\
\hline 985 & 25 & 985 & 25 & 985 & 25 & 985 & 25 & 986 & 25 & 100 & 25 \\
\hline 985 & 25 & 985 & 25 & 985 & 25 & 985 & 25 & 986 & 25 & 100 & 25 \\
\hline 985 & 25 & 985 & 25 & 985 & 25 & 985 & 25 & 986 & 25 & 100 & 25 \\
\hline 985 & 25 & 985 & 25 & 985 & 25 & 985 & 25 & 986 & 25 & 100 & 25 \\
\hline 985 & 25 & 985 & 25 & 985 & 25 & 985 & 25 & 986 & 25 & 100 & 25 \\
\hline 985 & 25 & 985 & 25 & 985 & 25 & 985 & 25 & 986 & 25 & 100 & 25 \\
\hline 985 & 25 & 985 & 25 & 985 & 25 & 985 & 25 & 986 & 25 & 100 & 25 \\
\hline 985 & 25 & 985 & 25 & 985 & 25 & 985 & 25 & 986 & 25 & 100 & 2 \\
\hline 985 & 25 & 985 & 25 & 985 & 25 & 985 & 25 & 986 & 25 & 100 & 25 \\
\hline 985 & 25 & 985 & 25 & 985 & 25 & 985 & 25 & 986 & 25 & 100 & 5 \\
\hline 985 & 25 & 985 & 25 & 985 & 25 & 985 & 25 & 986 & 25 & 100 & 25 \\
\hline 985 & 25 & 985 & 25 & 985 & 25 & 985 & 25 & 986 & 25 & 100 & \\
\hline 985 & 25 & 985 & 25 & 985 & 25 & 985 & 25 & 986 & 25 & 100 & 2 \\
\hline 985 & 25 & 985 & 25 & 985 & 25 & 985 & 25 & 986 & 25 & 100 & \\
\hline 985 & 25 & 985 & 25 & 985 & 25 & 985 & 25 & 986 & 25 & 100 & 25 \\
\hline 985 & 25 & 985 & 25 & 985 & 25 & 985 & 25 & 986 & 25 & 100 & \\
\hline 985 & 25 & 985 & 25 & 985 & 25 & 985 & 25 & 986 & 25 & 100 & 25 \\
\hline 985 & 25 & 985 & 25 & 985 & 25 & 985 & 25 & 986 & 25 & 100 & 5 \\
\hline 985 & 25 & 985 & 25 & 985 & 25 & 985 & 25 & 986 & 25 & 100 & 25 \\
\hline 985 & 25 & 985 & 25 & 985 & 25 & 985 & 25 & 986 & 25 & 100 & 25 \\
\hline 985 & 25 & 985 & 25 & 985 & 25 & 985 & 25 & 986 & 25 & 100 & \\
\hline 985 & 25 & 985 & 25 & 985 & 25 & 985 & 25 & 986 & 25 & 100 & 25 \\
\hline 985 & 25 & 985 & 25 & 985 & 25 & 985 & 25 & 986 & 25 & 100 & \\
\hline 985 & 25 & 985 & 25 & 985 & 25 & 985 & 25 & 986 & 25 & 100 & 25 \\
\hline 985 & 25 & 985 & 25 & 985 & 25 & 985 & 25 & 986 & 25 & 100 & \\
\hline 985 & 25 & 985 & 25 & 985 & 25 & 985 & 25 & 986 & 25 & 100 & 25 \\
\hline 985 & 25 & 985 & 25 & 985 & 25 & 985 & 25 & 986 & 25 & 100 & \\
\hline 985 & 25 & 985 & 25 & 985 & 25 & 985 & 25 & 986 & 25 & 100 & 25 \\
\hline 985 & 25 & 985 & 25 & 985 & 25 & 985 & 25 & 986 & 25 & 100 & \\
\hline 985 & 25 & 985 & 25 & 986 & 25 & 986 & 25 & 986 & 25 & 100 & 25 \\
\hline 985 & 25 & 985 & 25 & 986 & 25 & 986 & 25 & 986 & 25 & 100 & \\
\hline 985 & 25 & 985 & 25 & 986 & 25 & 986 & 25 & 986 & 25 & 100 & 25 \\
\hline 985 & 25 & 985 & 25 & 986 & 25 & 986 & 25 & 986 & 25 & 100 & \\
\hline 985 & 25 & 985 & 25 & 986 & 25 & 986 & 25 & 986 & 25 & 100 & 25 \\
\hline 985 & 25 & 985 & 25 & 986 & 25 & 986 & 25 & 986 & 25 & 100 & \\
\hline 985 & 25 & 985 & 25 & 986 & 25 & 986 & 25 & 986 & 25 & 100 & 25 \\
\hline 985 & 25 & 985 & 25 & 986 & 25 & 986 & 25 & 986 & 25 & 100 & \\
\hline 985 & 25 & 985 & 25 & 986 & 25 & 986 & 25 & 986 & 25 & 100 & 25 \\
\hline 985 & 25 & 985 & 25 & 986 & 25 & 900 & 25 & 986 & 25 & 100 & \\
\hline 985 & 25 & 985 & 25 & 986 & 25 & 800 & 25 & 986 & 25 & 100 & \\
\hline 985 & 25 & 985 & 25 & 986 & 25 & 700 & 25 & 986 & 25 & 100 & \\
\hline 985 & 25 & 985 & 25 & 986 & 25 & 600 & 25 & 986 & 25 & 100 & \\
\hline 985 & 25 & 985 & 25 & 986 & 25 & 500 & 25 & 986 & 25 & 100 & \\
\hline 985 & 25 & 985 & 25 & 986 & 25 & 400 & 25 & 986 & 25 & 100 & \\
\hline 985 & 25 & 985 & 25 & 986 & 25 & 300 & 25 & 986 & 25 & 100 & \\
\hline 985 & 25 & 985 & 25 & 986 & 25 & 200 & 25 & 986 & 25 & 100 & \\
\hline
\end{tabular}




\begin{tabular}{|c|c|c|c|c|c|c|c|c|c|c|c|}
\hline 986 & 25 & 100 & 25 & 987 & 25 & 930 & 25 & 988 & 25 & 988 & 25 \\
\hline 986 & 25 & 100 & 25 & 987 & 25 & 935 & 25 & 988 & 25 & 988 & 25 \\
\hline 986 & 25 & 100 & 25 & 987 & 25 & 940 & 25 & 988 & 25 & 988 & 25 \\
\hline 986 & 25 & 100 & 25 & 987 & 25 & 945 & 25 & 988 & 25 & 988 & 25 \\
\hline 986 & 25 & 100 & 25 & 987 & 25 & 950 & 25 & 988 & 25 & 988 & 25 \\
\hline 986 & 25 & 100 & 25 & 987 & 25 & 951 & 25 & 988 & 25 & 988 & 25 \\
\hline 986 & 25 & 100 & 25 & 987 & 25 & 952 & 25 & 988 & 25 & 988 & 25 \\
\hline 986 & 25 & 100 & 25 & 987 & 25 & 953 & 25 & 988 & 25 & 988 & 25 \\
\hline 986 & 25 & 100 & 25 & 987 & 25 & 954 & 25 & 988 & 25 & 988 & 25 \\
\hline 986 & 25 & 100 & 25 & 987 & 25 & 955 & 25 & 988 & 25 & 988 & 25 \\
\hline 986 & 25 & 100 & 25 & 987 & 25 & 956 & 25 & 988 & 25 & 950 & 25 \\
\hline 986 & 25 & 100 & 25 & 987 & 25 & 957 & 25 & 988 & 25 & 900 & 25 \\
\hline 986 & 25 & 100 & 25 & 987 & 25 & 958 & 25 & 988 & 25 & 850 & 25 \\
\hline 986 & 25 & 100 & 25 & 987 & 25 & 959 & 25 & 988 & 25 & 800 & 25 \\
\hline 986 & 25 & 100 & 25 & 987 & 25 & 960 & 25 & 988 & 25 & 750 & 25 \\
\hline 986 & 25 & 100 & 25 & 987 & 25 & 961 & 25 & 988 & 25 & 700 & 25 \\
\hline 986 & 25 & 100 & 25 & 987 & 25 & 962 & 25 & 988 & 25 & 650 & 25 \\
\hline 986 & 25 & 100 & 25 & 987 & 25 & 963 & 25 & 988 & 25 & 600 & 25 \\
\hline 986 & 25 & 100 & 25 & 987 & 25 & 964 & 25 & 988 & 25 & 550 & 25 \\
\hline 986 & 25 & 100 & 25 & 987 & 25 & 965 & 25 & 988 & 25 & 500 & 25 \\
\hline 986 & 25 & 100 & 25 & 987 & 25 & 966 & 25 & 988 & 25 & 475 & 25 \\
\hline 986 & 25 & 100 & 25 & 987 & 25 & 967 & 25 & 988 & 25 & 450 & 25 \\
\hline 986 & 25 & 100 & 25 & 987 & 25 & 968 & 25 & 988 & 25 & 425 & 25 \\
\hline 987 & 25 & 100 & 25 & 987 & 25 & 969 & 25 & 988 & 25 & 400 & 25 \\
\hline 987 & 25 & 100 & 25 & 987 & 25 & 970 & 25 & 988 & 25 & 375 & 25 \\
\hline 987 & 25 & 100 & 25 & 987 & 25 & 971 & 25 & 988 & 25 & 350 & 25 \\
\hline 987 & 25 & 100 & 25 & 987 & 25 & 972 & 25 & 988 & 25 & 325 & 25 \\
\hline 987 & 25 & 100 & 25 & 987 & 25 & 973 & 25 & 988 & 25 & 300 & 25 \\
\hline 987 & 25 & 100 & 25 & 987 & 25 & 974 & 25 & 988 & 25 & 275 & 25 \\
\hline 987 & 25 & 100 & 25 & 987 & 25 & 975 & 25 & 988 & 25 & 250 & 25 \\
\hline 987 & 25 & 100 & 25 & 987 & 25 & 976 & 25 & 988 & 25 & 225 & 25 \\
\hline 987 & 25 & 100 & 25 & 987 & 25 & 977 & 25 & 988 & 25 & 200 & 25 \\
\hline 987 & 25 & 100 & 25 & 987 & 25 & 978 & 25 & 988 & 25 & 175 & 25 \\
\hline 987 & 25 & 100 & 25 & 987 & 25 & 979 & 25 & 988 & 25 & 150 & 25 \\
\hline 987 & 25 & 100 & 25 & 987 & 25 & 980 & 25 & 988 & 25 & 125 & 25 \\
\hline 987 & 25 & 100 & 25 & 987 & 25 & 981 & 25 & 988 & 25 & 100 & 25 \\
\hline 987 & 25 & 100 & 25 & 987 & 25 & 982 & 25 & 988 & 25 & 75 & 25 \\
\hline 987 & 25 & 100 & 25 & 987 & 25 & 983 & 25 & 988 & 25 & 50 & 25 \\
\hline 987 & 25 & 100 & 25 & 987 & 25 & 984 & 25 & 988 & 25 & 50 & 25 \\
\hline 987 & 25 & 100 & 25 & 987 & 25 & 985 & 25 & 988 & 25 & 50 & 25 \\
\hline 987 & 25 & 100 & 25 & 987 & 25 & 986 & 25 & 988 & 25 & 50 & 25 \\
\hline 987 & 25 & 100 & 25 & 987 & 25 & 987 & 25 & 988 & 25 & 50 & 25 \\
\hline 987 & 25 & 150 & 25 & 987 & 25 & 987 & 25 & 988 & 25 & 50 & 25 \\
\hline 987 & 25 & 200 & 25 & 987 & 25 & 987 & 25 & 988 & 25 & 50 & 25 \\
\hline 987 & 25 & 250 & 25 & 987 & 25 & 987 & 25 & 988 & 25 & 50 & 25 \\
\hline 987 & 25 & 300 & 25 & 987 & 25 & 987 & 25 & 988 & 25 & 50 & 25 \\
\hline 987 & 25 & 350 & 25 & 987 & 25 & 987 & 25 & 988 & 25 & 50 & 25 \\
\hline 987 & 25 & 400 & 25 & 987 & 25 & 987 & 25 & 988 & 25 & 50 & 25 \\
\hline 987 & 25 & 450 & 25 & 987 & 25 & 987 & 25 & 988 & 25 & 50 & 25 \\
\hline 987 & 25 & 500 & 25 & 987 & 25 & 987 & 25 & 988 & 25 & 50 & 25 \\
\hline 987 & 25 & 550 & 25 & 987 & 25 & 987 & 25 & 988 & 25 & 50 & 25 \\
\hline 987 & 25 & 600 & 25 & 987 & 25 & 987 & 25 & 988 & 25 & 50 & 25 \\
\hline 987 & 25 & 650 & 25 & 988 & 25 & 988 & 25 & 988 & 25 & 50 & 25 \\
\hline 987 & 25 & 700 & 25 & 988 & 25 & 988 & 25 & 988 & 25 & 50 & 25 \\
\hline 987 & 25 & 750 & 25 & 988 & 25 & 988 & 25 & 988 & 25 & 50 & 25 \\
\hline 987 & 25 & 800 & 25 & 988 & 25 & 988 & 25 & 988 & 25 & 50 & 25 \\
\hline 987 & 25 & 810 & 25 & 988 & 25 & 988 & 25 & 988 & 25 & 50 & 25 \\
\hline 987 & 25 & 820 & 25 & 988 & 25 & 988 & 25 & 988 & 25 & 50 & 25 \\
\hline 987 & 25 & 830 & 25 & 988 & 25 & 988 & 25 & 988 & 25 & 50 & 25 \\
\hline 987 & 25 & 840 & 25 & 988 & 25 & 988 & 25 & 988 & 25 & 50 & 25 \\
\hline 987 & 25 & 850 & 25 & 988 & 25 & 988 & 25 & 988 & 25 & 50 & 25 \\
\hline 987 & 25 & 860 & 25 & 988 & 25 & 988 & 25 & 988 & 25 & 50 & 25 \\
\hline 987 & 25 & 870 & 25 & 988 & 25 & 988 & 25 & 988 & 25 & 50 & 25 \\
\hline 987 & 25 & 880 & 25 & 988 & 25 & 988 & 25 & 988 & 25 & 50 & 25 \\
\hline 987 & 25 & 890 & 25 & 988 & 25 & 988 & 25 & 988 & 25 & 50 & 25 \\
\hline 987 & 25 & 900 & 25 & 988 & 25 & 988 & 25 & 988 & 25 & 50 & 25 \\
\hline 987 & 25 & 905 & 25 & 988 & 25 & 988 & 25 & 988 & 25 & 50 & 25 \\
\hline 987 & 25 & 910 & 25 & 988 & 25 & 988 & 25 & 988 & 25 & 50 & 25 \\
\hline 987 & 25 & 915 & 25 & 988 & 25 & 988 & 25 & 988 & 25 & 50 & 25 \\
\hline 987 & 25 & 920 & 25 & 988 & 25 & 988 & 25 & 988 & 25 & 50 & 25 \\
\hline 987 & 25 & 925 & 25 & 988 & 25 & 988 & 25 & 988 & 25 & 50 & 25 \\
\hline
\end{tabular}




\begin{tabular}{|c|c|c|c|c|c|c|c|c|c|c|c|}
\hline 988 & 25 & 50 & 25 & 989 & 25 & 50 & 25 & 990 & 25 & 929 & 25 \\
\hline 988 & 25 & 50 & 25 & 989 & 25 & 50 & 25 & 990 & 25 & 932 & 25 \\
\hline 988 & 25 & 50 & 25 & 989 & 25 & 50 & 25 & 990 & 25 & 935 & 25 \\
\hline 988 & 25 & 50 & 25 & 989 & 25 & 60 & 25 & 990 & 25 & 938 & 25 \\
\hline 988 & 25 & 50 & 25 & 989 & 25 & 70 & 25 & 990 & 25 & 941 & 25 \\
\hline 988 & 25 & 50 & 25 & 989 & 25 & 80 & 25 & 990 & 25 & 944 & 25 \\
\hline 988 & 25 & 50 & 25 & 989 & 25 & 90 & 25 & 990 & 25 & 947 & 25 \\
\hline 988 & 25 & 50 & 25 & 989 & 25 & 100 & 25 & 990 & 25 & 950 & 25 \\
\hline 988 & 25 & 50 & 25 & 989 & 25 & 110 & 25 & 990 & 25 & 951 & 25 \\
\hline 988 & 25 & 50 & 25 & 989 & 25 & 120 & 25 & 990 & 25 & 952 & 25 \\
\hline 989 & 25 & 50 & 25 & 989 & 25 & 130 & 25 & 990 & 25 & 953 & 25 \\
\hline 989 & 25 & 50 & 25 & 989 & 25 & 140 & 25 & 990 & 25 & 954 & 25 \\
\hline 989 & 25 & 50 & 25 & 989 & 25 & 150 & 25 & 990 & 25 & 955 & 25 \\
\hline 989 & 25 & 50 & 25 & 989 & 25 & 160 & 25 & 990 & 25 & 956 & 25 \\
\hline 989 & 25 & 50 & 25 & 989 & 25 & 170 & 25 & 990 & 25 & 957 & 25 \\
\hline 989 & 25 & 50 & 25 & 989 & 25 & 180 & 25 & 990 & 25 & 958 & 25 \\
\hline 989 & 25 & 50 & 25 & 989 & 25 & 190 & 25 & 990 & 25 & 959 & 25 \\
\hline 989 & 25 & 50 & 25 & 989 & 25 & 200 & 25 & 990 & 25 & 960 & 25 \\
\hline 989 & 25 & 50 & 25 & 989 & 25 & 210 & 25 & 990 & 25 & 961 & 25 \\
\hline 989 & 25 & 50 & 25 & 989 & 25 & 220 & 25 & 990 & 25 & 962 & 25 \\
\hline 989 & 25 & 50 & 25 & 989 & 25 & 230 & 25 & 990 & 25 & 963 & 25 \\
\hline 989 & 25 & 50 & 25 & 989 & 25 & 240 & 25 & 990 & 25 & 964 & 25 \\
\hline 989 & 25 & 50 & 25 & 989 & 25 & 250 & 25 & 990 & 25 & 965 & 25 \\
\hline 989 & 25 & 50 & 25 & 989 & 25 & 260 & 25 & 990 & 25 & 966 & 25 \\
\hline 989 & 25 & 50 & 25 & 989 & 25 & 270 & 25 & 990 & 25 & 967 & 25 \\
\hline 989 & 25 & 50 & 25 & 989 & 25 & 280 & 25 & 990 & 25 & 968 & 25 \\
\hline 989 & 25 & 50 & 25 & 989 & 25 & 290 & 25 & 990 & 25 & 969 & 25 \\
\hline 989 & 25 & 50 & 25 & 989 & 25 & 300 & 25 & 990 & 25 & 970 & 25 \\
\hline 989 & 25 & 50 & 25 & 989 & 25 & 310 & 25 & 990 & 25 & 971 & 25 \\
\hline 989 & 25 & 50 & 25 & 989 & 25 & 320 & 25 & 990 & 25 & 972 & 25 \\
\hline 989 & 25 & 50 & 25 & 989 & 25 & 330 & 25 & 990 & 25 & 973 & 25 \\
\hline 989 & 25 & 50 & 25 & 989 & 25 & 340 & 25 & 990 & 25 & 974 & 25 \\
\hline 989 & 25 & 50 & 25 & 989 & 25 & 350 & 25 & 990 & 25 & 975 & 25 \\
\hline 989 & 25 & 50 & 25 & 989 & 25 & 375 & 25 & 990 & 25 & 976 & 25 \\
\hline 989 & 25 & 50 & 25 & 989 & 25 & 400 & 25 & 990 & 25 & 977 & 25 \\
\hline 989 & 25 & 50 & 25 & 989 & 25 & 425 & 25 & 990 & 25 & 978 & 25 \\
\hline 989 & 25 & 50 & 25 & 989 & 25 & 450 & 25 & 990 & 25 & 979 & 25 \\
\hline 989 & 25 & 50 & 25 & 989 & 25 & 475 & 25 & 990 & 25 & 980 & 25 \\
\hline 989 & 25 & 50 & 25 & 989 & 25 & 500 & 25 & 990 & 25 & 981 & 25 \\
\hline 989 & 25 & 50 & 25 & 990 & 25 & 525 & 25 & 990 & 25 & 982 & 25 \\
\hline 989 & 25 & 50 & 25 & 990 & 25 & 550 & 25 & 990 & 25 & 983 & 25 \\
\hline 989 & 25 & 50 & 25 & 990 & 25 & 575 & 25 & 990 & 25 & 984 & 25 \\
\hline 989 & 25 & 50 & 25 & 990 & 25 & 600 & 25 & 990 & 25 & 985 & 25 \\
\hline 989 & 25 & 50 & 25 & 990 & 25 & 625 & 25 & 990 & 25 & 986 & 25 \\
\hline 989 & 25 & 50 & 25 & 990 & 25 & 650 & 25 & 990 & 25 & 987 & 25 \\
\hline 989 & 25 & 50 & 25 & 990 & 25 & 675 & 25 & 990 & 25 & 988 & 25 \\
\hline 989 & 25 & 50 & 25 & 990 & 25 & 700 & 25 & 990 & 25 & 989 & 25 \\
\hline 989 & 25 & 50 & 25 & 990 & 25 & 725 & 25 & 990 & 25 & 990 & 25 \\
\hline 989 & 25 & 50 & 25 & 990 & 25 & 750 & 25 & 990 & 25 & 990 & 25 \\
\hline 989 & 25 & 50 & 25 & 990 & 25 & 775 & 25 & 990 & 25 & 990 & 25 \\
\hline 989 & 25 & 50 & 25 & 990 & 25 & 800 & 25 & 990 & 25 & 990 & 25 \\
\hline 989 & 25 & 50 & 25 & 990 & 25 & 825 & 25 & 990 & 25 & 990 & 25 \\
\hline 989 & 25 & 50 & 25 & 990 & 25 & 850 & 25 & 990 & 25 & 990 & 25 \\
\hline 989 & 25 & 50 & 25 & 990 & 25 & 875 & 25 & 990 & 25 & 990 & 25 \\
\hline 989 & 25 & 50 & 25 & 990 & 25 & 878 & 25 & 990 & 25 & 990 & 25 \\
\hline 989 & 25 & 50 & 25 & 990 & 25 & 881 & 25 & 990 & 25 & 990 & 25 \\
\hline 989 & 25 & 50 & 25 & 990 & 25 & 884 & 25 & 990 & 25 & 990 & 25 \\
\hline 989 & 25 & 50 & 25 & 990 & 25 & 887 & 25 & 990 & 25 & 990 & 25 \\
\hline 989 & 25 & 50 & 25 & 990 & 25 & 890 & 25 & 990 & 25 & 990 & 25 \\
\hline 989 & 25 & 50 & 25 & 990 & 25 & 893 & 25 & 990 & 25 & 990 & 25 \\
\hline 989 & 25 & 50 & 25 & 990 & 25 & 896 & 25 & 990 & 25 & 990 & 25 \\
\hline 989 & 25 & 50 & 25 & 990 & 25 & 899 & 25 & 990 & 25 & 990 & 25 \\
\hline 989 & 25 & 50 & 25 & 990 & 25 & 902 & 25 & 990 & 25 & 990 & 25 \\
\hline 989 & 25 & 50 & 25 & 990 & 25 & 905 & 25 & 990 & 25 & 990 & 25 \\
\hline 989 & 25 & 50 & 25 & 990 & 25 & 908 & 25 & 990 & 25 & 990 & 25 \\
\hline 989 & 25 & 50 & 25 & 990 & 25 & 911 & 25 & 990 & 25 & 990 & 25 \\
\hline 989 & 25 & 50 & 25 & 990 & 25 & 914 & 25 & 990 & 25 & 990 & 25 \\
\hline 989 & 25 & 50 & 25 & 990 & 25 & 917 & 25 & 990 & 25 & 990 & 25 \\
\hline 989 & 25 & 50 & 25 & 990 & 25 & 920 & 25 & 990 & 25 & 990 & 25 \\
\hline 989 & 25 & 50 & 25 & 990 & 25 & 923 & 25 & 990 & 25 & 990 & 25 \\
\hline 989 & 25 & 50 & 25 & 990 & 25 & 926 & 25 & 990 & 25 & 990 & 25 \\
\hline
\end{tabular}




\begin{tabular}{|c|c|c|c|c|c|c|c|c|c|c|c|}
\hline 990 & 25 & 990 & 25 & 990 & 25 & 990 & 25 & 990 & 25 & 990 & 25 \\
\hline 990 & 25 & 990 & 25 & 990 & 25 & 990 & 25 & 990 & 25 & 990 & 25 \\
\hline 990 & 25 & 990 & 25 & 990 & 25 & 990 & 25 & 990 & 25 & 990 & 25 \\
\hline 990 & 25 & 990 & 25 & 990 & 25 & 990 & 25 & 990 & 25 & 990 & 25 \\
\hline 990 & 25 & 990 & 25 & 990 & 25 & 990 & 25 & 990 & 25 & 990 & 25 \\
\hline 990 & 25 & 990 & 25 & 990 & 25 & 990 & 25 & 990 & 25 & 990 & 25 \\
\hline 990 & 25 & 990 & 25 & 990 & 25 & 990 & 25 & 990 & 25 & 990 & 25 \\
\hline 990 & 25 & 990 & 25 & 990 & 25 & 990 & 25 & 990 & 25 & 990 & 25 \\
\hline 990 & 25 & 990 & 25 & 990 & 25 & 990 & 25 & 990 & 25 & 990 & 25 \\
\hline 990 & 25 & 990 & 25 & 990 & 25 & 990 & 25 & 990 & 25 & 990 & 25 \\
\hline 990 & 25 & 990 & 25 & 990 & 25 & 990 & 25 & 990 & 25 & 990 & 25 \\
\hline 990 & 25 & 990 & 25 & 990 & 25 & 990 & 25 & 990 & 25 & 990 & 25 \\
\hline 990 & 25 & 990 & 25 & 990 & 25 & 990 & 25 & 990 & 25 & 990 & 25 \\
\hline
\end{tabular}


Appendix B: Feeder Load Profile Data

\section{The following is the feeder load profile that was used in the Distribution Circuit module via the Load_Data.txt file:}

\begin{tabular}{|c|c|c|c|c|c|c|c|c|c|c|}
\hline ! R1 & R2 & R3 & R4 & R5 & R6 & R7 & R8 & R9 & R10 & R11 \\
\hline 794 & 1296 & 5184 & 51840 & 1728 & 1605 & 3988 & 2592 & 498 & 5184 & 741 \\
\hline 789 & 1280 & 4800 & 51840 & 1734 & 1605 & 3957 & 2529 & 494 & 5033 & 730 \\
\hline 784 & 1264 & 4469 & 51840 & 1740 & 1605 & 3927 & 2469 & 489 & 4891 & 720 \\
\hline 780 & 1249 & 4181 & 51840 & 1745 & 1605 & 3898 & 2411 & 484 & 4756 & 710 \\
\hline 775 & 1234 & 3927 & 51840 & 1751 & 1605 & 3869 & 2356 & 480 & 4629 & 701 \\
\hline 770 & 1220 & 3703 & 51840 & 1757 & 1605 & 3840 & 2304 & 476 & 4508 & 691 \\
\hline 766 & 1206 & 3503 & 51840 & 1763 & 1605 & 3812 & 2254 & 471 & 4393 & 682 \\
\hline 761 & 1192 & 3323 & 51840 & 1769 & 1605 & 3784 & 2206 & 467 & 4284 & 673 \\
\hline 757 & 1178 & 3161 & 51840 & 1775 & 1481 & 3757 & 2160 & 463 & 4181 & 665 \\
\hline 752 & 1165 & 3014 & 51840 & 1781 & 1481 & 3729 & 2116 & 467 & 4082 & 656 \\
\hline 748 & 1152 & 2880 & 51840 & 1788 & 1481 & 3703 & 2074 & 471 & 3988 & 648 \\
\hline 744 & 1139 & 2757 & 51840 & 1794 & 1481 & 3677 & 2033 & 476 & 3898 & 640 \\
\hline 740 & 1127 & 2645 & 51840 & 1800 & 1481 & 3651 & 1994 & 480 & 3812 & 632 \\
\hline 735 & 1115 & 2541 & 51840 & 1806 & 1481 & 3625 & 1956 & 484 & 3729 & 625 \\
\hline 731 & 1103 & 2445 & 51840 & 1813 & 1481 & 3600 & 1920 & 489 & 3651 & 617 \\
\hline 727 & 1091 & 2356 & 51840 & 1819 & 1481 & 3575 & 1885 & 494 & 3575 & 610 \\
\hline 723 & 1080 & 2274 & 51840 & 1825 & 1481 & 3551 & 1851 & 498 & 3503 & 603 \\
\hline 719 & 1069 & 2197 & 51840 & 1832 & 1481 & 3527 & 1819 & 503 & 3433 & 596 \\
\hline 715 & 1058 & 2125 & 25920 & 1838 & 1481 & 3503 & 1788 & 508 & 3366 & 589 \\
\hline 711 & 1047 & 2057 & 25920 & 1845 & 1481 & 3479 & 1757 & 513 & 3302 & 582 \\
\hline 707 & 1037 & 1994 & 25920 & 1851 & 1481 & 3456 & 1728 & 518 & 3240 & 576 \\
\hline 703 & 1027 & 1934 & 25920 & 1858 & 1481 & 3456 & 1700 & 516 & 3180 & 570 \\
\hline 700 & 1016 & 1878 & 25920 & 1865 & 3456 & 3456 & 1672 & 513 & 3123 & 563 \\
\hline 696 & 1007 & 1825 & 25920 & 1871 & 3456 & 3456 & 1646 & 511 & 3067 & 557 \\
\hline 692 & 997 & 1775 & 25920 & 1878 & 3456 & 3456 & 1620 & 508 & 3014 & 551 \\
\hline 688 & 987 & 1728 & 25920 & 1885 & 3456 & 3456 & 1595 & 506 & 2962 & 546 \\
\hline 685 & 978 & 1683 & 25920 & 1892 & 3456 & 3456 & 1571 & 503 & 2912 & 540 \\
\hline 681 & 969 & 1728 & 25920 & 1899 & 3456 & 3456 & 1547 & 501 & 2864 & 534 \\
\hline 678 & 960 & 1775 & 25920 & 1906 & 3456 & 3456 & 1525 & 498 & 2817 & 529 \\
\hline 674 & 951 & 1825 & 25920 & 1913 & 3456 & 3456 & 1503 & 496 & 2772 & 524 \\
\hline 671 & 943 & 1878 & 25920 & 1920 & 3456 & 3479 & 1481 & 494 & 2728 & 518 \\
\hline 667 & 934 & 1934 & 25920 & 1927 & 3456 & 3503 & 1460 & 491 & 2686 & 513 \\
\hline 664 & 926 & 1994 & 25920 & 1934 & 3456 & 3527 & 1440 & 489 & 2645 & 508 \\
\hline 660 & 918 & 2057 & 25920 & 1949 & 3456 & 3551 & 1420 & 487 & 2605 & 503 \\
\hline 657 & 909 & 2125 & 25920 & 1964 & 1364 & 3575 & 1401 & 484 & 2566 & 498 \\
\hline 654 & 902 & 2197 & 25920 & 1979 & 1364 & 3600 & 1382 & 482 & 2529 & 494 \\
\hline 650 & 894 & 2274 & 17280 & 1994 & 1364 & 3625 & 1364 & 480 & 2492 & 489 \\
\hline 647 & 886 & 2356 & 17280 & 1920 & 1364 & 3651 & 1346 & 478 & 2457 & 484 \\
\hline 644 & 879 & 2445 & 17280 & 1851 & 1364 & 3677 & 1329 & 476 & 2422 & 480 \\
\hline 641 & 871 & 2541 & 17280 & 1788 & 1364 & 3703 & 1312 & 473 & 2389 & 476 \\
\hline 638 & 864 & 2645 & 17280 & 1728 & 1364 & 3729 & 1296 & 471 & 2356 & 471 \\
\hline 635 & 857 & 2757 & 17280 & 1672 & 1364 & 3757 & 1280 & 469 & 2325 & 467 \\
\hline 631 & 850 & 2645 & 17280 & 1620 & 1364 & 3784 & 1264 & 467 & 2294 & 463 \\
\hline 628 & 843 & 2541 & 17280 & 1571 & 1364 & 3812 & 1249 & 465 & 2264 & 459 \\
\hline 625 & 836 & 2445 & 17280 & 1525 & 1364 & 3840 & 1234 & 463 & 2234 & 455 \\
\hline 622 & 829 & 2356 & 17280 & 1481 & 1364 & 3703 & 1220 & 461 & 2206 & 451 \\
\hline 619 & 823 & 2274 & 17280 & 1440 & 1364 & 3575 & 1206 & 459 & 2178 & 682 \\
\hline 616 & 816 & 2197 & 12960 & 1401 & 1364 & 3456 & 1192 & 457 & 2151 & 687 \\
\hline 613 & 810 & 2125 & 12960 & 1364 & 1364 & 3345 & 1178 & 455 & 2125 & 691 \\
\hline 611 & 804 & 2057 & 12960 & 1329 & 1364 & 3240 & 1165 & 453 & 2099 & 696 \\
\hline 608 & 798 & 1994 & 12960 & 1296 & 1728 & 3142 & 1152 & 451 & 2074 & 701 \\
\hline 605 & 791 & 1934 & 12960 & 1264 & 1728 & 3049 & 1139 & 449 & 2049 & 705 \\
\hline 602 & 785 & 1878 & 12960 & 1234 & 1728 & 2962 & 1127 & 447 & 2025 & 710 \\
\hline 599 & 780 & 1825 & 12960 & 1206 & 1728 & 2880 & 1115 & 445 & 2002 & 715 \\
\hline 597 & 774 & 1775 & 12960 & 1178 & 1728 & 2802 & 1103 & 443 & 1979 & 720 \\
\hline 594 & 768 & 1728 & 12960 & 1152 & 1728 & 2728 & 1091 & 441 & 1956 & 725 \\
\hline 591 & 762 & 1683 & 12960 & 1127 & 1728 & 2658 & 1080 & 439 & 1934 & 730 \\
\hline 588 & 757 & 1641 & 12960 & 1103 & 1728 & 2592 & 1069 & 437 & 1913 & 735 \\
\hline 586 & 751 & 1600 & 12960 & 1080 & 1728 & 2529 & 1058 & 436 & 1892 & 741 \\
\hline 583 & 746 & 1561 & 12960 & 1058 & 1728 & 2469 & 1047 & 434 & 1871 & 746 \\
\hline
\end{tabular}

